\title{
DESCRIPTION OF DATA-COLLECTION SYSTEM AND SYNOPSIS OF SELECTED HYDROLOGIC DATA FOR SOLDIER CREEK BASIN, KANSAS
}

U.S. GEOLOGLA' SURVEY

Open-File Report 78-678

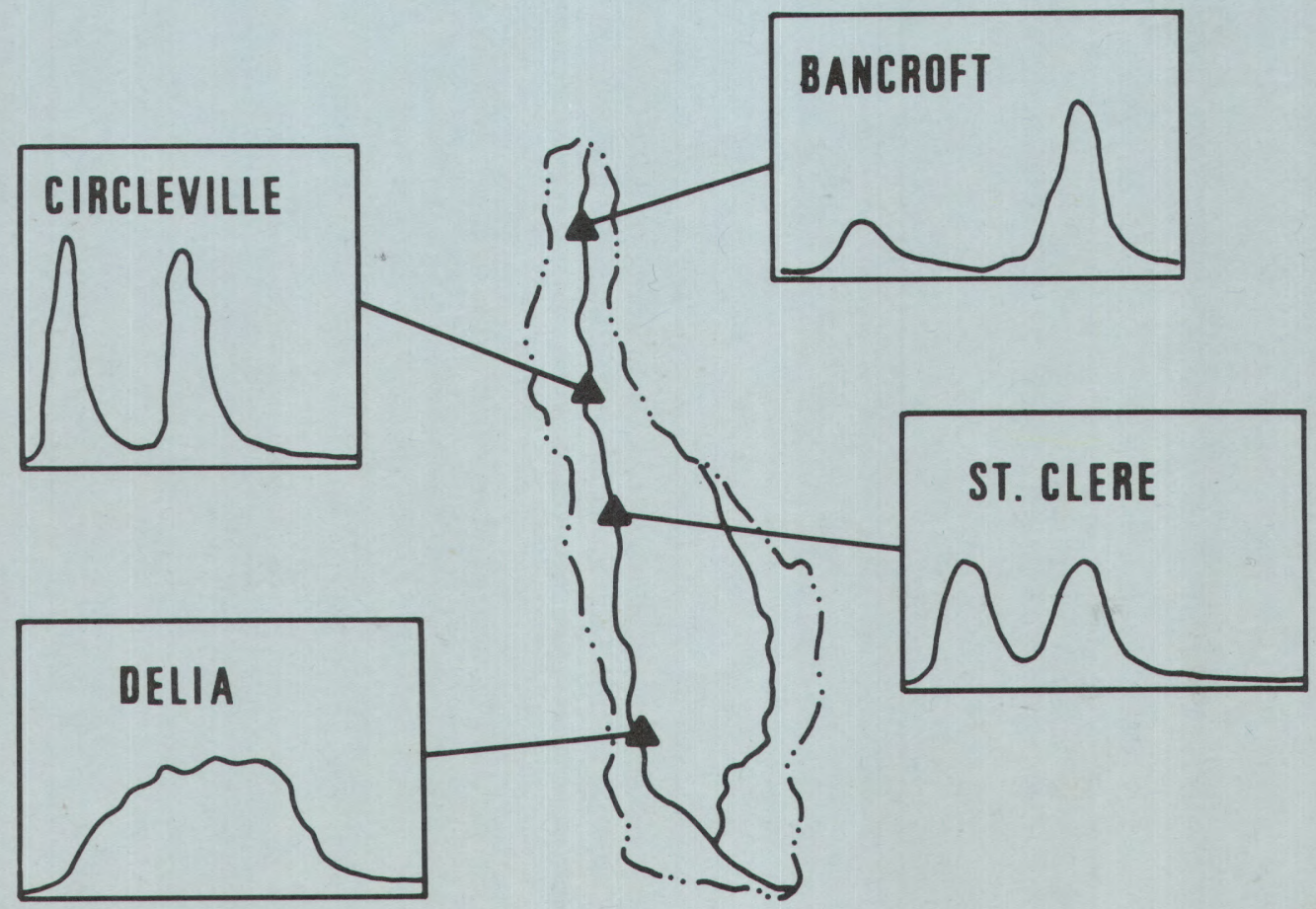

Prepared in cooperation with

the Kansas Water Resources Board 

UNITED STATES

DEPARTMENT OF THE INTERIOR

GEOLOGICAL SURVEY

DESCRIPTION OF DATA-COLLECTION SYSTEM AND

SYNOPSIS OF SELECTED HYDROLOGIC DATA

FOR SOLDIER CREEK BASIN, KANSAS

By W. J. Carswell, Jr.

Open-FIle Report 78-678

Prepared in cooperation with the

Kansas Water Resources Board

Lawrence, Kansas

July 1978 


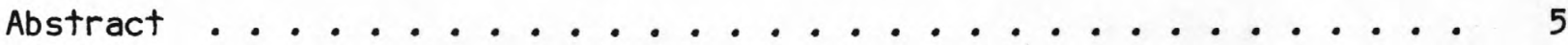

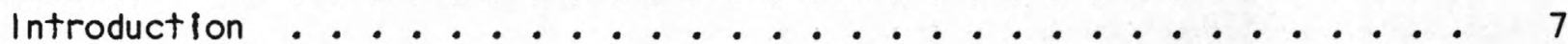

Conversion table ....................... 7

Acknow ledgments ............................. 8

General description of basin . . . . . . . . . . . . 8

Physical features .................... 8

Climate ......................... . . . . 9

Description of data-collection system . . . . . . . . . . . 9

Ralnfall gages..................... 9

Streamflow gages ...................... 14

Water-quality sampling sites .............. 14

Seepage-salinity investigations .............. . . 15

Ground-water observation wells ............ 20

Data for selected events of rainfall and peak flow . . . . . . . 20

Supplementary data . . . . . . . . . . . . . . 58

Water quallity ......................... 58

Seepage-salinity investigations ............. 58

Ground water .................. . . 58

Summary . . . . . . . . . . . . . . . . . . . 64

Selected references... . . . . . . . . . . . . . . . 64

Appendix A . . . . . . . . . . . . . . . . . . . 65

Definition of terms for basin characteristics . . . . . . 65

Streamflow station descriptions and selected basin

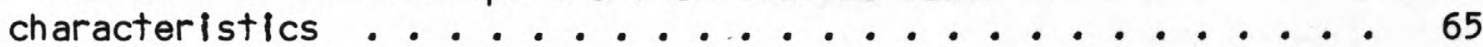

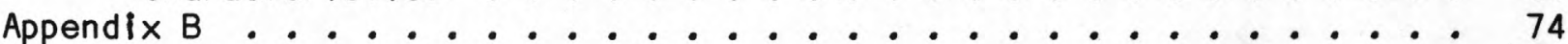

Ground-water well descriptions ............. 74 


\section{ILLUSTRATIONS}

Figure

Page

1. Maps showing location of Soldier Creek basin and streamflow-gaging stations .............. . . 6

2. Photographs showing dual-purpose installation for collecting river-stage and ralnfall data ........... 10

3. Schematic diagrams showing location of equipment for collecting river-stage and rainfall data . . . . . . . . 10

4. Map showing general location of rainfall gages In and near Soldler Creek basin ................ 11

5. Diagram showing numbering system used for location of rainfall gages, observation wells, and seepage-

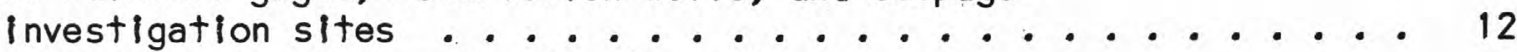

6. Map showing location of main-stem sites of seepage investigations ................. . . 19

7. Map showing location of water wells at the upper streamflow-gaging sites .................. 21

Figures 8-29--Maps and graphs showing:

8. Distribution of rainfall June 4, 1964, and resulting stream discharge . . . . . . . . . . . 29

9. Distribution of rainfall June 11-12, 1964, and resulting stream discharge ................. 30

10. Distribution of rainfall June 14-15, 1964,

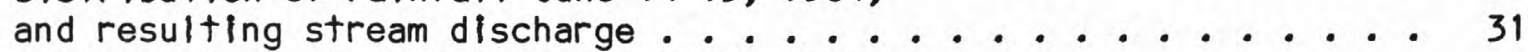

11. Distribution of rainfall June $22-23,1964$, and resulting stream discharge .............. 32

12. Distribution of rainfall June $27-28,1965$, and resulting stream discharge ................... 35

13. Distribution of rainfall September 20-21, 1965, and resulting stream discharge ............... 36

14. Distribution of rainfall June 4-5, 1967, and resulting stream discharge............... 37

15. Distribution of rainfall June 20-21, 1967, and resulting stream discharge.................... 38

16. Distribution of rainfall July $23-24,1968$, and resulting stream discharge .................. 39

17. Distribution of rainfall August 9-10, 1968, and resulting stream discharge............ 40

18. Distribution of rainfall April 3-4, 1969, and resulting stream discharge.............. 42

19. Distribution of rainfall April 26, 1969,

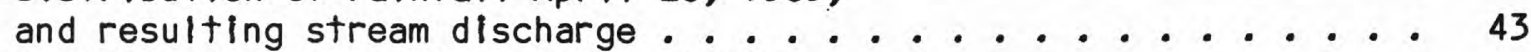

20. Distribution of rainfall May 7, 1969, and resulting stream discharge............... 44

21. Distribution of rainfall May 10, 1970, and resulting stream discharge ................ 
22. Distribution of rainfall August 2-3, 1972, and resulting stream discharge .............. . . 4 46

23. Distribution of rainfall September 6-7, 1972, and resulting stream discharge............... 47

24. Distribution of rainfall May 6-7, 1973, and resulting stream discharge.............. 48

25. Distribution of rainfall August 7-8, 1973, and resulting stream discharge .............. 51

26. Distribution of rainfall September 26-27, 1973, and resulting stream discharge .............. . . 52

27. Distribution of rainfall October 10-11, 1973, and resulting stream discharge .............. . . 54

28. Distribution of rainfall April 20, 1974, and resulting stream discharge .............. 56

29. Distribution of rainfall April 29, 1974, and resulting stream discharge ...............

30. Graph showing water discharges at main-stem sites along Soldier Creek ....................

31. Graph showing dissolved-solids concentrations at main-stem sites along Soldier Creek . . . . . . . . . 62

32. Hydrographs of water levels in observation well 8-13E-ICCD4 and in Soldier Creek near St. Clere gaging station

TABLES

Table

1. List of ralnfall observers ............... . . 13

2. List of seepage-investigation sites ............. 16

3. Soldier Creek ralnfall and peak-flow data . . . . . . . . . . . . 23

4. Chemical analyses of water samples for Soldier Creek

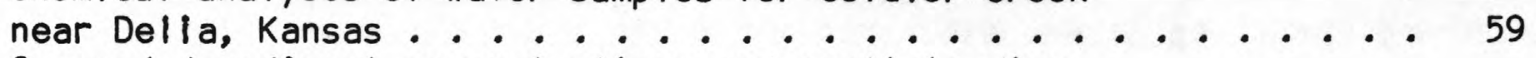

5. Suspended-sediment concentrations and particle-size distribution at selected sites on Soldier Creek during

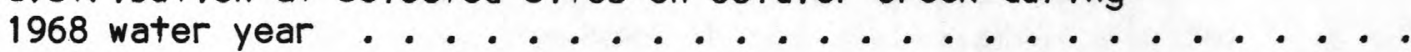




\section{DESCRIPTION OF DATA-COLLECTION SYSTEM AND \\ SYNOPSIS OF SELECTED HYDROLGOIC DATA \\ FOR SOLDIER CREEK BASIN, KANSAS}

W. J. Carswell, Jr.

\section{ABSTRACT}

Soldier Creek basin is a long, narrow basin encompassing an area of about 290 square miles almost directly north of Topeka, Kansas. A wide range of hydrologic data has been collected in the basin since the spring of 1964 . These data include rainfall, stream discharge, sediment concentrations, chemical quality of water, and ground-water altitudes.

The data collection system consists of 7 recording streamflow stations, 5 recording rainfall stations, 51 nonrecording rainfall stations, and 31 groundwater observation wells. Sediment and chemical quality of water samples were collected intermittently at selected sites.

A synopsis of the time and space distribution of rainfall and peak flow are provided in graphic and tabular form for selected events of rainfall and peak flow. Representative data concerning the chemical quality of water and the fluvial sediment also are included. Selected ground-water and seepageinvesitgation data are depicted graphically. 


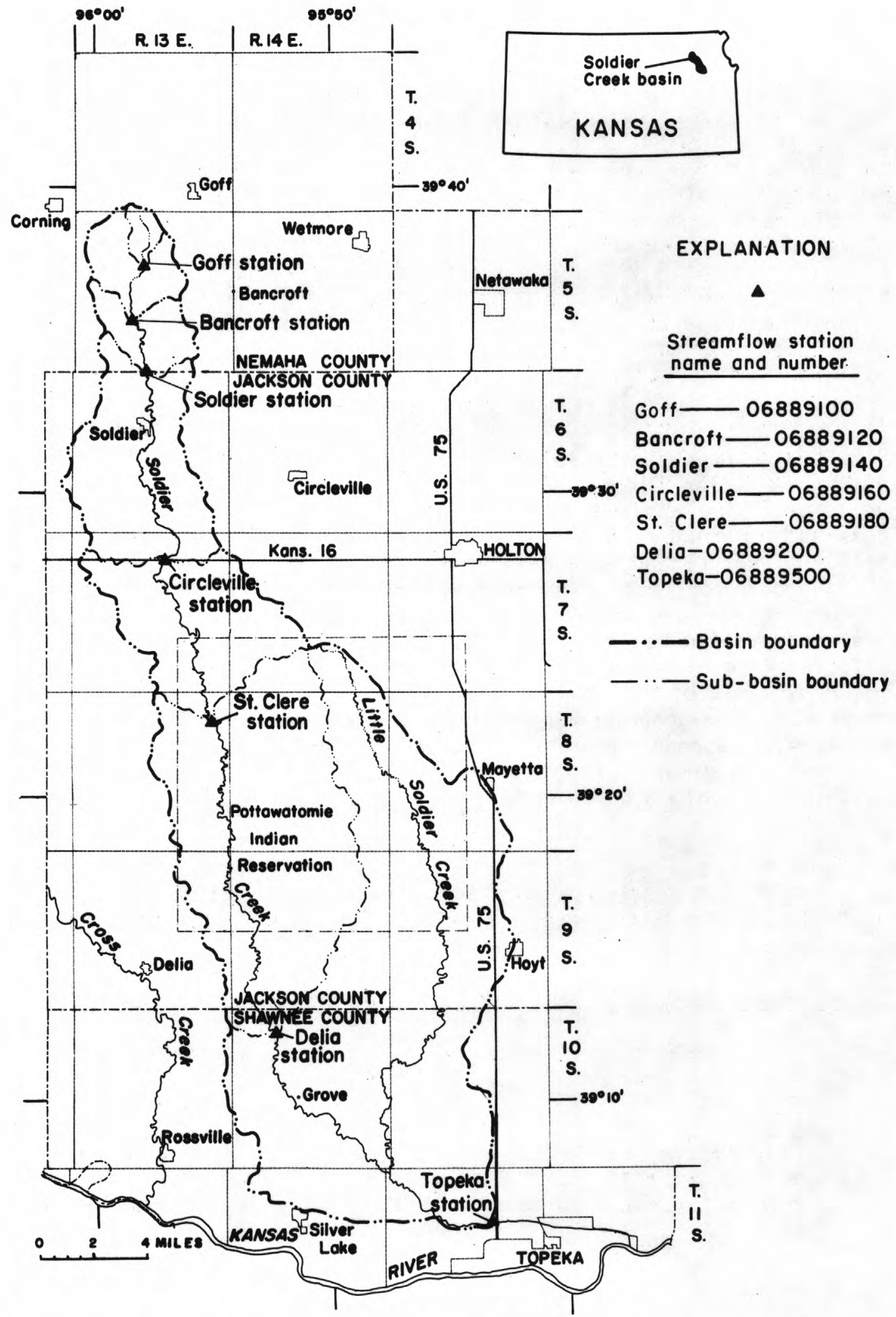

Figure 1.--Location of Soldier Creek basin and streamflow-gaging stations. 


\section{INTRODUCTION}

In the spring of 1964, the U.S. Geological Survey in cooperation with the Kansas Water Resources Board designed and Installed a hydrologic-datacollection system in Soldier Creek basin. The basic system was designed primarlly to obtain detalled rainfall and runoff data. This basic system was expanded to include collection of supplementary data that would provide additional insight concerning the basin's general hydrologic framework. The basic system is composed of: (1) 7 recording streamflow stations, as shown in figure 1; (2) 5 recording rainfall stations; and (3) 51 nonrecording rainfall stations located within and adjacent to the basin. The remainder of the system includes intermittent chemical quality of water and sediment-sampling sites and 31 ground-water observation wells. A comprehensive compllation of hydrologic data collected in the basin is avallable to users in computerreadable form from a nine channel magnetic tape (Carswell, in preparation). If a potential user does not have access to computer faclilities, tables of selected data can be obtained by request from the nearest U.S. Geological Survey, Water Resources Division, office.

The twofold purpose of this report is: (1) to describe the data-collection system and (2) to display selected data collected in the basin in such a way that a potential user can determine if the data avallable for the basin will fit his needs. The major thrust of this report will coincide with that of the system, that is major emphasis on the rainfall and flood-hydrograph data, supplemented with data for other aspects of the hydrologic setting. The data avallable for Soldier Creek basin will provide a wealth of information for water-resources studies including, but not limited to, rainfall-runoff modeling, flood routing, sediment yleid, and the relation of surface water to ground water.

\section{CONVERSION TABLE}

For users of this report who may wish to convert American Customary units to metric units, the following factors and standard abbreviations are given:

\begin{tabular}{lc} 
American Customary unit & Multiply by \\
\cline { 2 - 2 } Inch (in) & 25.4 \\
foot $(\mathrm{ft})$ & .3048 \\
mile $(\mathrm{ml})$ & 1.609 \\
acre & 4,047 \\
square mile $\left(\mathrm{ml}^{2}\right)$ & 2.590 \\
foot per mile $(\mathrm{ft} / \mathrm{ml})$ & .1894 \\
cubic foot per second $\left(f t^{3} / \mathrm{s}\right)$ & .02832
\end{tabular}

Metric unit

millimeters (mm)

meter (m)

kilometers $(\mathrm{km})$

square meters $\left(\mathrm{m}^{2}\right)$

square kilometers $\left(\mathrm{km}^{2}\right)$

$\mathrm{meter} / \mathrm{kllometer}$

cubic meter per second $\left(\mathrm{m}^{3} / \mathrm{s}\right)$ 


\section{ACKNOWLEDGMENTS}

Streamflow, rainfall, and sediment data used in this report were collected by the U.S. Geological Survey through cooperative programs with the Kansas Water Resources Board and the Corps of Engineers, U.S. Department of the Army.

Data on chemical quality of water were collected through the cooperative program between the U.S. Geological Survey and the Kansas Department of Health and Environment.

Observation wells for monitoring water-level changes were installed with assistance from the Kansas Geological Survey as part of the statewide cooperative ground-water investigation program.

\section{GENERAL DESCRIPTION OF BASIN}

\section{Physical Features}

The Soldier Creek basin (fig. 1) lies in the Dissected Till Plains and Attenuated Drift Border sections of the Central Lowlands physiographic province. Rock formations that crop out in the basin are principally shales and limestones of Pennsylvanian and Permian age.* Pleistocene glaciation has affected the stream patterns and has left till and outwash accumulations that are the predominant surface material over much of the basin. A thin cover of windblown silt or loess overlies the till in some areas, but the thicker deposits occur in the northern part of the basin. Alluvial sand, gravel, silt, and clay of Pleistocene to Holocene age, which are the major source of ground water, underlie the valleys of Soldier Creek and its major tributaries. For additional discussion concerning the geology and ground-water resources of the basin and surrounding area, the reader is directed to Walters (1953) and Ward (1974).

The Soldier Creek valley is about $48 \mathrm{miles}$ long and ranges from about one-half mile wide in the upper reaches to about 2 miles wide near its entrance to the Kansas River valley. The lower reach of Soldier Creek flows in the valley of the Kansas River for about 10 miles.

The topography of the basin is undulating with relief commonly greater than 100 feet. The soil on the level alluvium in the valley consists of sand, gravel, silt, and clay. The side slopes are composed of stony or gravelly soils, whereas the uplands have a rather heavy-textured, nearly impermeable soil. Land use in the basin is almost exclusively agricultural with 54 percent cropland, 38 percent pasture, and 8 percent forested area and other uses.

* The stratigraphic nomenclature used in this report is that of the U.S. Geological Survey and may differ somewhat from that of the Kansas Geological Survey. 
Soldier Creek basin lies in northeast Kansas where the climate is characterized by warm to hot summers, cold winters, moderate surface winds, and average annual precipitation of 35 inches. Warm, moist air from the Gulf of Mexico is the primary source of moisture for the precipitation that falls on the basin. Approximately 70 percent of the average annual precipitation falls during the growing season, April through September, with June having the maximum monthly average.

\section{DESCRIPTION OF DATA-COLLECTION SYSTEM}

Rainfall Gages

Data on the quantity and areal distribution of rainfall during the period of study were collected at 56 sites in and adjacent to the Soldier Creek basin. Five of the rainfall-data sites are equipped with recording gages located at streamflow-gaging stations.

These synchronous rainfall and river-stage gages, as shown in figure 2, are located at the gaging stations near Goff, Bancroft, Soldier, Circleville, and St. Clere. The equipment for collecting the rainfall data is shown in figure 3. A funnel 7.51 inches in diameter, similar to the type used by the National Weather Service (National Oceanic and Atmospheric Administration, U.S. Department of Commerce), provides the desired ratio with the diameter of the collecting well. The funnel is supported approximately 11 feet above the ground by a 3/4-inch diameter galvanized intake pipe. Copper tubing connects the intake pipe to the collector well. The well is a 3-inch diameter galvanized pipe, 54 inches long, capped at one end, and is attached to the instrument shelf. A beaded chain attached to a solid plastic float activates a digital-punch recorder (15-minute punch interval).

Both digital-punch recorders for collecting rainfall data and river-stage data at each station are operated by the same timer to eliminate time differences. Prior to April 1969, the rainfall at these gage installations was recorded to an accuracy of 0.02 inch. Beginning in April 1969, rainfall was recorded to an accuracy of 0.01 inch.

The 51 nonrecording rainfall gages were installed at selected sites, as shown in figure 4. These rainfall gages were operated by local observers on a daily basis, except during the winter months (November through March). All observers were instructed to read the gage as near to 7:00 a.m. as possible. The rainfall was to be recorded to the nearest 0.01 inch for the preceding 24 hours on the day that the gage was read. Weather Service form 612-14 was used by the observers to record the rainfall. A spot comparison of observer readings with each other and with the recording cages indicated some inconsistencies. It was apparent that some readings were not made at 7:00 a.m., and occasionally some rainfall was recorded on the wrong day. 


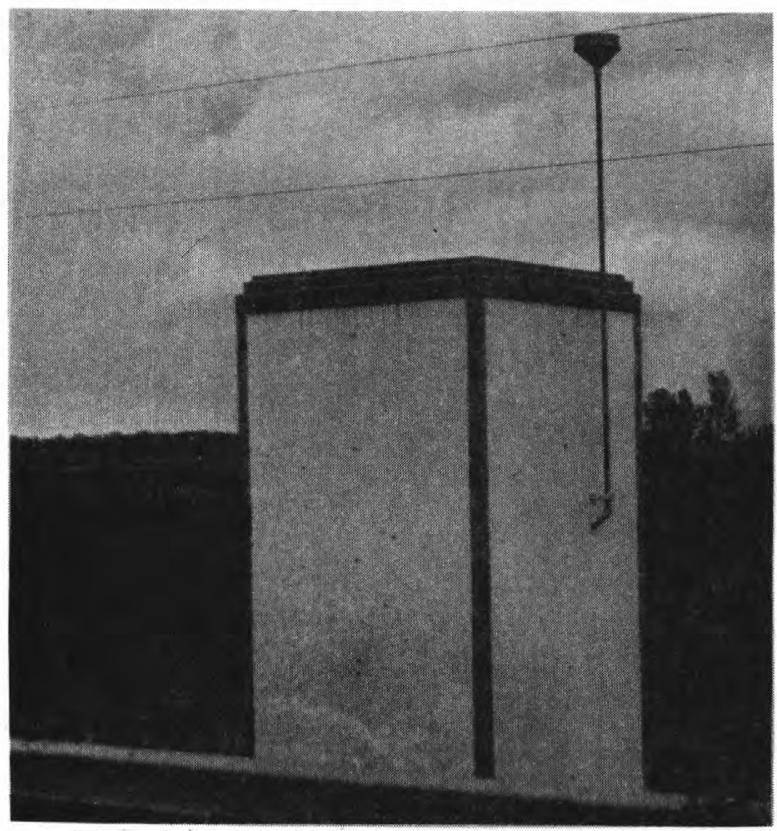

Figure 2.--Dual-purpose installation for collecting river-stage and rainfall data.

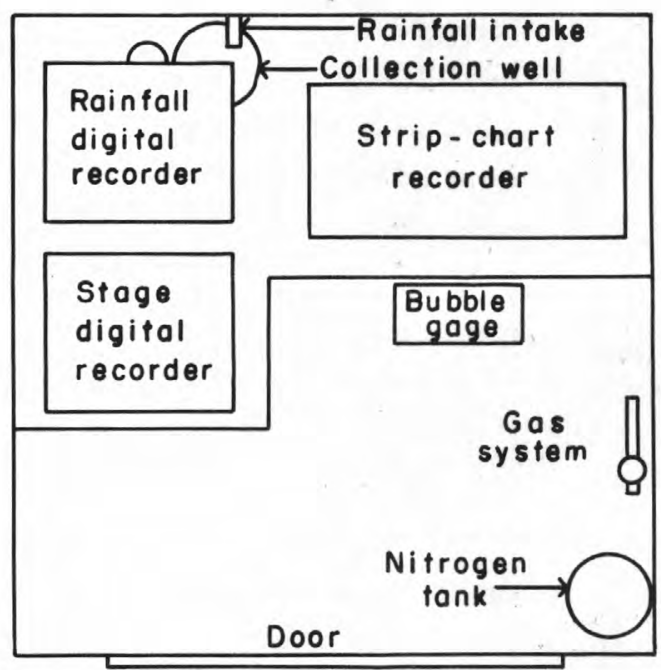

Top View
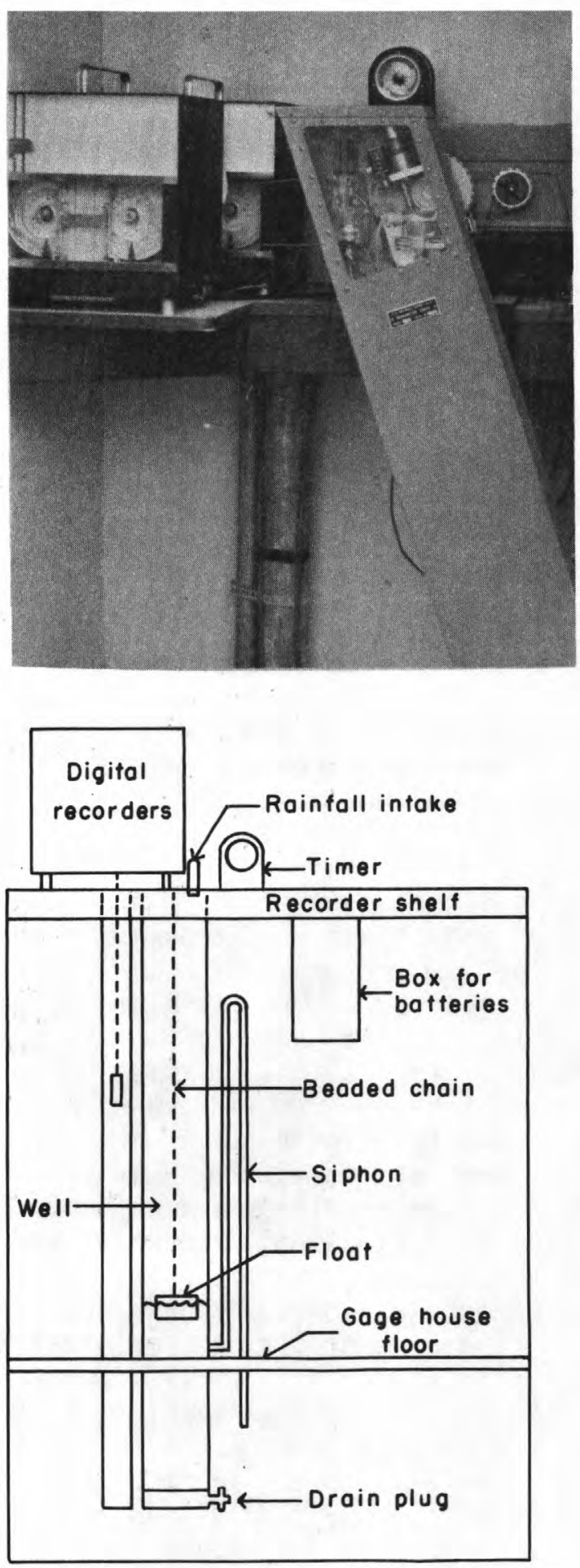

\section{Front View}

(bubble gage and strip-chart recorder not shown)

Figure 3.--Location of equipment in an installation for collecting river-stage and rainfall data. 


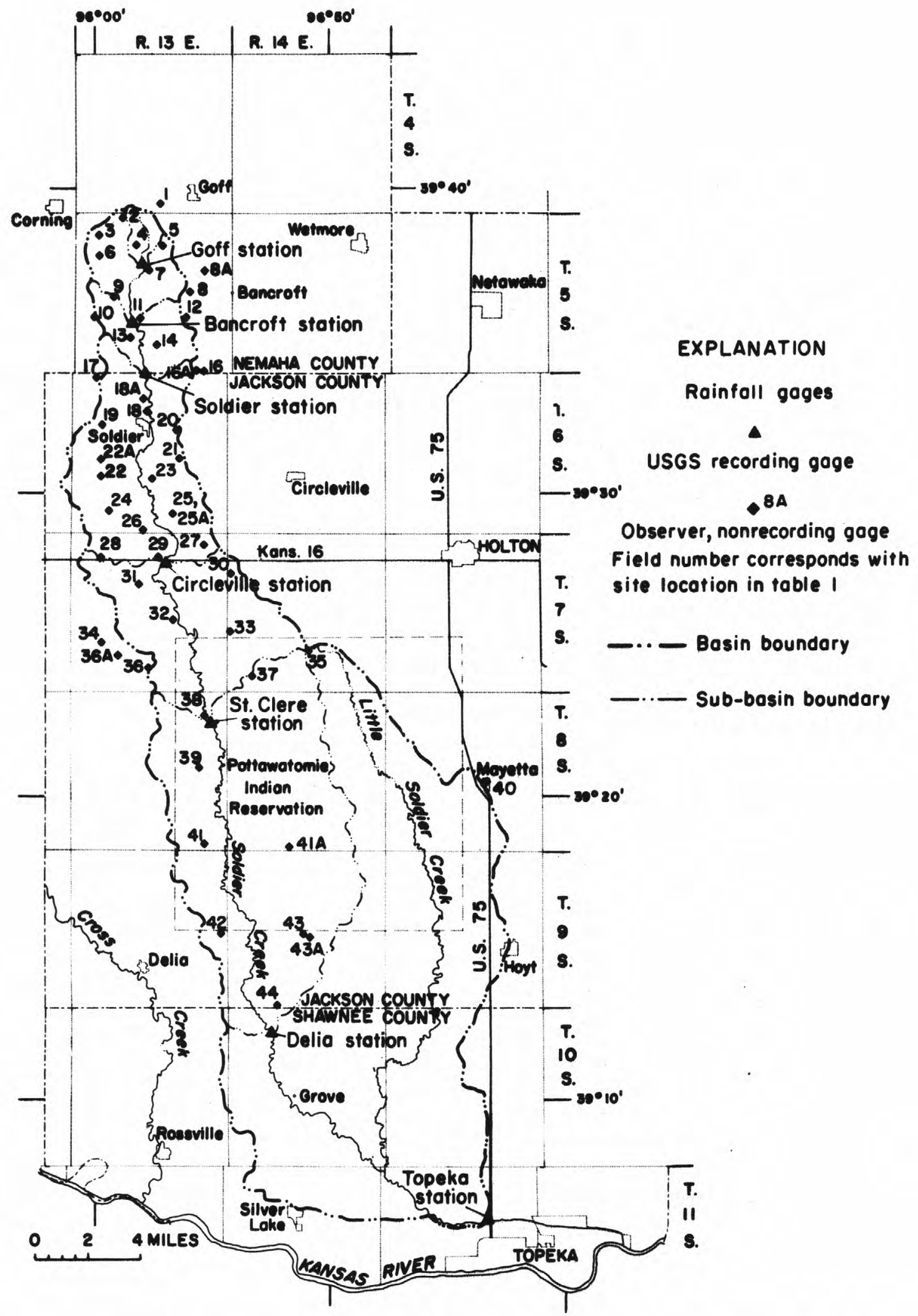

Figure 4.--General location of rainfall gages in and near Soldier Creek basin. 
Table 1 gives a list of the nonrecording rain gages showing the assigned field number corresponding to figure 4, observer's name, site location, and inclusive dates of available data for each gage.

The location of rainfall-observation sites are described according to the Bureau of Land Management's system of land subdivision. The first number indicates the township; the second number, the range east of the Sixth Principal Meridian; and the third number, the section. The first letter designates the quarter section (160-acretract); the second letter, the quarter-quarter section (40-acretract); and the third letter, when used, designates the quarter-quarterquarter section (10-acre tract). The 160-acre, 40-acre, and 10-acre tracts are designated $A, B, C$, and $D$ in a counterclockwise direction beginning in the nor theast quadrant. For example, the location of rainfall-observation site 5-13E-9BA is

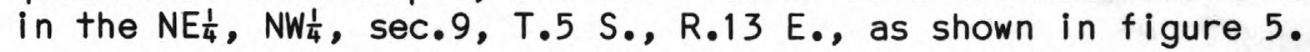

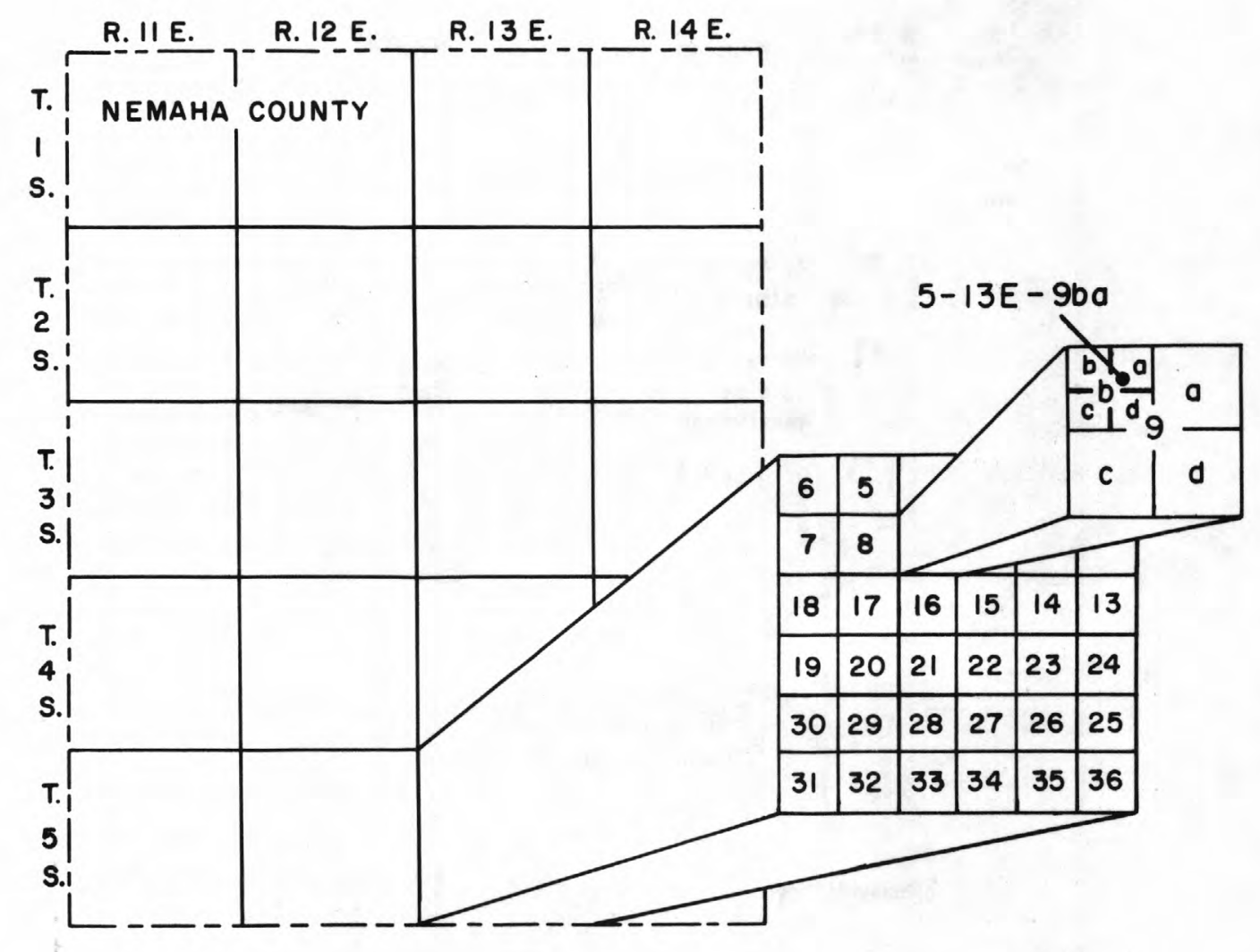

Figure 5.--Numbering system used for location of rainfall gages, observation wells, and seepage-investigations sites. 
Field

number

1

2

3

4

5

6

\section{Observer}

M. Powell

B. H. Engleken

W. F. El senbarth

L. Jerome

J. Mulroy

D. Alexander

E. Hermesch

C. Alley

I. Bloom

D. Feldkamp

C. J. Meyer

F. H. Swartz

R. Watk ins

L. L. Barnes

L. E. Watkins

W. J. Leuthold

T. W. Mohringer

B. Coe

E. I. Schrieber

S. Franz

E. Cordell

E. Freel

A. Staeh I I

O. H. Moulden

L. Stanton

C. Rieschick

K. Rieschick

H. Fisher

R. Dugan

E. Claycamp

H. Wolcott

F. Fisher

J. Cowger

G. Beightel

W. Stewart

A. Cowger

L. Valburg

W. C. Eby

M. McClane

R. May

M. Dibbern

H. Brucken

F. Kruger

E. W. Houck

I. James

L. V. Keller

M. Wyatt

D. Wulfkuhle

M. Bail ley

V. E. Albright

J. Bahner
Site location

4-13E-34CB

$5-13 E-5 A B$

5-13E-6DA

$5-13 E-9 B A$

5-13E-1 OBB

$5-13 E-7 A D$

5-13E-16AB

5-13E-14CD

5-13E-14AA

5-13E-20BA

5-13E-19DC

5-13E-21DC

5-13E-23CC

5-13E-28CB

5-13E-27CC

$5-13 E-35 C D$

5-13E-35DD

$6-13 E-6 A A$

6-13E-9AD

6-13E-4DD

$6-13 E-8 C C$

6-13E-1 4BB

6-13.E-23BB

$6-13 \mathrm{E}-20 \mathrm{CC}$

6-13E-20BB

$6-13 \mathrm{E}-22 \mathrm{CC}$

6-13E-32BA

6-13E-34AA

6-13E-34DA

6-13E-33DD

$7-13 E-1 B C$

$7-13 E-5 C C$

$7-13 E-3 C D$

7-14E-7BC

7-13E-9DC

$7-13 E-22 A A$

$7-14 \mathrm{E}-19 \mathrm{CC}$

$7-13 \mathrm{E}-29 \mathrm{BB}$

7-14E-27BC

7-13E-33AA

7-13E-29DA

7-14E-31AD

8-13E-1CC

8-13E-14DD

8-15E-22AD

$8-13 E-36 C C$

8-14E-33CD

9-13E-24AB

$9-14 \mathrm{E}-22 \mathrm{BB}$

9-14E-21AA

9-14E-32DD
Record avallable

May 1964 to May 1974

May 1964 to Sept. 1976

May 1964 to Aug. 1969

Apr. 1964 to Sept. 1976

May 1964 to Sept. 1976

May 1964 to Sept. 1976

Apr. 1964 to Sept. 1965

May 1964 to June 1969

July 1969 to Sept. 1976

May 1964 to Sept. 1976

May 1964 to Sept. 1976

May 1964 to Nov. 1964

May 1964 + Sept. 1976

May 1964 to Oct. 1972

May 1964 to Sept. 1976

May 1964 to Sept. 1964

Oct. 1964 to Sept. 1976

May 1964 to May 1976

May 1964 to Oct. 1964

Apr. 1965 to July 1965

May 1964 to Aug. 1968

May 1964 to Sept. 1976

May 1964 to Sept. 1976

May 1964 to May 1969

June 1969 to Sept. 1976

May 1964 to Sept. 1976

May 1964 to June 1969

May 1964 to May 1974

May 1973 to Oct. 1975

June 1964 to Sept. 1976

May 1964 to July 1968

May 1964 to Sept. 1976

Apr. 1964 to Sept. 1976

May 1964 to Sept. 1976

May 1964 to Oct. 1971

May 1964 to Sept. 1976

May 1964 to May 1976

May 1964 to Oct. 1968

May 1964 to Oct. 1973

June 1964 to Aug. 1966

May 1967 to May 1968

May 1964 to Sept. 1976

Apr. 1964 to Sept. 1976

May 1964 to Sept. 1976

July 1965

May 1964 to Aug. 1965

May 1968 to Sept. 1973

May 1964 to Sept. 1976

May 1964 to Oct. 1964

July 1969 to Sept. 1976

Apr. 1965 to June 1969

May 1964 to Aug. 1966 
Streamflow data summarized in this report were obtained at seven streamflowgaging stations having contributing drainage areas ranging in size from 2.06 to 290 square miles. Two gaging stations in existence prior to the investigation provided long-term records for the basin. Five additional gaging stations were installed to provide streamflow records from various sizes of contributing drainage areas.

The earllest records are from the station on Soldier Creek near Topeka, established in May 1929, and from the station on Soldier Creek near Della, established in October 1958. The remaining five gaging stations on Soldier Creek were installed in March 1964. Each station is equipped with a digital-punch recorder (15-minute interval) and a graphical recorder driven by a 50-foot-range bubble gage for monitoring the river stage.

Streamflow data collected at each gaging station consists of stage records and current-meter measurements of discharge at specific stages. Rating curves and tables for each station are prepared showing the stage-discharge relation defined by the discharge measurements. Extensions to the rating curves, necessary to express discharge greater than measured, are made on the basis of indirect measurements of peak discharge, velocity-area studies, or a logarithmic plotting of the stage-discharge relation. The mean daily discharges and peak discharges are computed from recorded gage heights and rating tables.

In appendix A, a detalled description of each gaging station is given that includes: station name and number (assigned by U.S. Geological Survey); location by latitude and longltude, land subdivision, geographlc description, and distance above mouth in stream miles; drainage area; date of installation and operating agency; type of gage and recorder installation; gage datum above NGVD (National Geodetic Vertical Datum) of 1929; channel-control conditions; historical record of operating conditions, floods, and regulation; and selected climatic and physlographic parameters.

\section{Water-Quality Sampling Sites}

The water-quality data collected in Soldier Creek basin is not as detailed as that for rainfall and streamflow. However, a significant amount of waterquality data has been collected in the basin. The water-quality information includes chemical and physical characteristics, and fluvial-sediment data.

The chemical-quality information includes concentrations of individual dissolved constituents, expressed in milligrams per $1 /$ ter $(\mathrm{mg} / \mathrm{L})$, and other characteristics such as hardness, sodium-absorption ratio, specific conductance, and $\mathrm{pH}$. The Soldier Creek near Della station is the only site at which chemicalquality data were routinely collected, and records from November 1965 to September 1975 are published in the annual Water Resources Data for Kansas (U.S. Geological Survey, (964-76). 
Fluvial-sediment data collected during the study at all streamflow-gaging sites except Topeka include the water discharges, concentrations of suspended sediment, and the particle-size distribution of both bed materlal and suspended sediment. Standard techniques of the U.S. Geological Survey were used to collect and analyze the sediment data. Suspended-sediment samples were collected using depth-integrating and single-stage samplers.

\section{SEEPAGE-SAL INITY INVESTIGATIONS}

As an additional aid to better define the general hydrologic framework, two seepage-salinity investigations have been conducted in the Soldier Creek basin. A list of the measurement and sampling sites, their location, river mlle, and drainage area is given in table 2. Fleld numbers shown in the first column preceded by an $R$ denote a main-stem slte, and numbers preceded by a $T$ denote a tributary site. For the user's convenience, the site location is in the same form as that used for the rainfall-observation sites. The station number is given in parenthesis below the site location if the site. is a regular streamflow station. The latitude and longltude are given for each site to correspond with the location given in that form on the Soldier Creek data tape (Carswell, in preparation). The river mile and dralnage area listed for malnstem sites correspond to the location at which the data were collected. If the site is on a tributary, the river mile is given for the point at which the tributary joins the main stem. The dralnage area given in the table is the total area contributing from the tributary, with the data collected as close to the mouth as possible. Data obtained during the seepage investigations on November 7, 1963, and July 22, 1964, Include water discharge and chemlcal analysis of the water sample collected at the time of each measurement. The location of main-stem sites of the seepage investigations is shown in figure 6 . 
Table 2.--List of seepage-investigation sites.

\begin{tabular}{|c|c|c|c|c|c|}
\hline $\begin{array}{l}\text { Fleld } \\
\text { number }\end{array}$ & $\begin{array}{c}\text { Site location } \\
\text { (station number) } \\
\end{array}$ & Latitude - & Long itude & $\begin{array}{l}\text { River } \\
\text { mile } \\
\end{array}$ & $\begin{array}{l}\text { Dralnage } \\
\text { area }\left(\mathrm{m} l^{2}\right)\end{array}$ \\
\hline R1 & $\begin{array}{l}5-13 E-16 A B B \\
(06889100)\end{array}$ & $39^{\circ} 27^{\prime} 27^{\prime \prime}$ & $95^{\circ} 57^{\prime} 57^{\prime \prime}$ & 71.9 & 2.06 \\
\hline T1 & $5-13 E-9 D D C$ & $39^{\circ} 37^{\prime} 29^{\prime \prime}$ & $95^{\circ} 57^{\prime} 26^{\prime \prime}$ & 71.7 & .80 \\
\hline T2 & $5-13 E-17 D D C$ & $39^{\circ} 36^{\prime} 43^{\prime \prime}$ & $95^{\circ} 58^{\prime} 22^{\prime \prime}$ & 70.0 & 4.11 \\
\hline R2 & $\begin{array}{l}5-13 E-28 B B A \\
(06889120)\end{array}$ & $39^{\circ} 35^{\prime} 42^{\prime \prime}$ & $95^{\circ} 58^{\prime} 17^{\prime \prime}$ & 68.7 & 10.5 \\
\hline T3 & $5-13 E-29 A A A$ & $39^{\circ} 35^{\prime} 38^{\prime \prime}$ & $95^{\circ} 58^{\prime} 22^{\prime \prime}$ & 68.4 & 1.67 \\
\hline R3 & $\begin{array}{l}6-13 E-4 A A B \\
(06889140)\end{array}$ & $39^{\circ} 33^{\prime} 57^{\prime \prime}$ & $95^{\circ} 57^{\prime} 45^{\prime \prime}$ & 65.7 & 16.9 \\
\hline R4 & $6-13 E-10 B B B$ & $39^{\circ} 33^{\prime} 02^{\prime \prime}$ & $95^{\circ} 57^{\prime} 35^{\prime \prime}$ & 64.5 & 20.2 \\
\hline T4 & $6-13 E-4 D D C$ & $39^{\circ} 33^{\prime} 08^{\prime \prime}$ & $95^{\circ} 57^{\prime} 52^{\prime \prime}$ & 64.2 & 1.93 \\
\hline T5 & $6-13 E-9 D A B$ & $39^{\circ} 32^{\prime} 36^{\prime \prime}$ & $95^{\circ} 57^{\prime} 52^{\prime \prime}$ & 63.5 & 1.68 \\
\hline R5 & $6-13 E-15 B B B$ & $39^{\circ} 32^{\prime} 10^{\prime \prime}$ & $95^{\circ} 57^{\prime} 35^{\prime \prime}$ & 62.9 & 24.4 \\
\hline R6 & $6-13 E-16 D A D$ & $39^{\circ} 31^{\prime} 37^{\prime \prime}$ & $95^{\circ} 57^{\prime} 43^{\prime \prime}$ & 62.1 & 25.6 \\
\hline T6 & $6-13 E-16 D B D$ & $39^{\circ} 31^{\prime} 37^{\prime \prime}$ & $95^{\circ} 58^{\prime} 00^{\prime \prime}$ & 61.9 & 2.83 \\
\hline $\mathrm{T7}$ & $6-13 E-21 C B B$ & $39^{\circ} 30^{\prime} 51^{\prime \prime}$ & $95^{\circ} 58^{\prime} 42^{\prime \prime}$ & 60.3 & 2.21 \\
\hline $\mathrm{R7}$ & $6-13 E-28 A B A$ & $39^{\circ} 30^{\prime} 25^{\prime \prime}$ & $95^{\circ} 58^{\prime} 00^{\prime \prime}$ & 59.5 & 32.7 \\
\hline T8 & $6-13 E-28 A A A$ & $39^{\circ} 30^{\prime} 25^{\prime \prime}$ & $95^{\circ} 57^{\prime} 43^{\prime \prime}$ & 59.2 & 1.71 \\
\hline $\mathrm{R} 8$ & $6-13 E-28 D A A$ & $39^{\circ} 29^{\prime} 59^{\prime \prime}$ & $95^{\circ} 57^{\prime} 43^{\prime \prime}$ & 59.0 & 34.8 \\
\hline T9 & $6-13 E-33 A A A$ & $39^{\circ} 29^{\prime} 33^{\prime \prime}$ & $95^{\circ} 57^{\prime} 43^{\prime \prime}$ & 58.3 & 4.95 \\
\hline R9 & $6-13 E-34 B B B$ & $39^{\circ} 29^{\prime} 33^{\prime \prime}$ & $95^{\circ} 57^{\prime} 35^{\prime \prime}$ & 58.2 & 40.1 \\
\hline T11 & $7-13 E-2 C B B$ & $39^{\circ} 28^{\prime} 14^{\prime \prime}$ & $95^{\circ} 56^{\prime} 28^{\prime \prime}$ & 55.7 & 1.83 \\
\hline T10 & $7-13 E-4 A D A$ & $39^{\circ} 28^{\prime} 27^{\prime \prime}$ & $95^{\circ} 57^{\prime} 43^{\prime \prime}$ & 55.2 & 1.85 \\
\hline R10 & $\begin{array}{l}7-13 E-10 A B B \\
(06889160)\end{array}$ & $39^{\circ} 27^{\prime} 47^{\prime \prime}$ & $95^{\circ} 57^{\prime} 00^{\prime \prime}$ & 55.2 & 49.3 \\
\hline
\end{tabular}


Table 2.--List of seepage-investigation sites (continued).

\begin{tabular}{|c|c|c|c|c|c|}
\hline $\begin{array}{l}\text { Fleld } \\
\text { number }\end{array}$ & $\begin{array}{c}\text { Site location } \\
\text { (station number) } \\
\end{array}$ & Latitude - & Long itude & $\begin{array}{l}\text { River } \\
\text { milee } \\
\end{array}$ & $\begin{array}{l}\text { Dralnage, } \\
\left.\text { area }(\mathrm{m}\}^{2}\right)\end{array}$ \\
\hline $\mathrm{T} 12$ & $7-13 E-3 C D D$ & $39^{\circ} 27^{\prime} 54^{\prime \prime}$ & $95^{\circ} 57^{\prime} 10^{\prime \prime}$ & 55.1 & .66 \\
\hline R11 & $7-13 E-10 D C C$ & $39^{\circ} 27^{\prime} 02^{\prime \prime}$ & $95^{\circ} 57^{\prime} 01^{\prime \prime}$ & 53.4 & 51.5 \\
\hline T13 & $7-13 E-16 A A D$ & $39^{\circ} 26^{\prime} 49^{\prime \prime}$ & $95^{\circ} 57^{\prime} 43^{\prime \prime}$ & 52.8 & 4.43 \\
\hline T14 & $7-13 E-16 C D C$ & $39^{\circ} 26^{\prime} 10^{\prime \prime}$ & $95^{\circ} 58^{\prime} 25^{\prime \prime}$ & 51.6 & 2.26 \\
\hline $\mathrm{R} 12$ & $7-13 E-23 B B B$ & $39^{\circ} 26^{\prime} 03^{\prime \prime}$ & $95^{\circ} 56^{\prime} 28^{\prime \prime}$ & 51.5 & 60.4 \\
\hline T15 & $7-13 E-23 B C C$ & $39^{\circ} 25^{\prime} 43^{\prime \prime}$ & $95^{\circ} 56^{\prime} 28^{\prime \prime}$ & 50.9 & .29 \\
\hline T16 & $7-13 E-26 A A A$ & $39^{\circ} 25^{\prime} 11^{\prime \prime}$ & $95^{\circ} 55^{\prime} 29^{\prime \prime}$ & 48.9 & 11.1 \\
\hline T17 & $7-13 E-36 B B C$ & $39^{\circ} 24^{\prime} 12^{\prime \prime}$ & $95^{\circ} 55^{\prime} 21^{\prime \prime}$ & 47.3 & .20 \\
\hline $\mathrm{R} 13$ & $7-13 E-35 D D A$ & $39^{\circ} 23^{\prime} 39^{\prime \prime}$ & $95^{\circ} 55^{\prime} 29^{\prime \prime}$ & 46.3 & 75.9 \\
\hline T18 & $7-13 E-36 C C C$ & $39^{\circ} 23^{\prime} 32^{\prime \prime}$ & $95^{\circ} 55^{\prime} 21^{\prime \prime}$ & 46.2 & 1.18 \\
\hline $\mathrm{R} 14$ & $\begin{array}{l}8-13 E-12 B A B \\
(06889180)\end{array}$ & $39^{\circ} 22^{\prime} 33^{\prime \prime}$ & $95^{\circ} 55^{\prime} 05^{\prime \prime}$ & 44.5 & 80.5 \\
\hline T19 & $8-13 E-12 B B C$ & $39^{\circ} 22^{\prime} 27^{\prime \prime}$ & $95^{\circ} 55^{\prime} 21^{\prime \prime}$ & 44.2 & 1.49 \\
\hline $\mathrm{T} 20$ & $8-13 E-12 A A A$ & $39^{\circ} 22^{\prime} 33^{\prime \prime}$ & $95^{\circ} 54^{\prime} 23^{\prime \prime}$ & 43.0 & 1.05 \\
\hline R14A & $8-13 E-13 D D B$ & $39^{\circ} 21^{\prime} 02^{\prime \prime}$ & $95^{\circ} 54^{\prime} 31^{\prime \prime}$ & 40.0 & 93.5 \\
\hline $\mathrm{T} 21$ & $8-13 E-13 C C C$ & $39^{\circ} 20^{\prime} 55^{\prime \prime}$ & $95^{\circ} 55^{\prime} 21^{\prime \prime}$ & 39.1 & 3.22 \\
\hline $\mathrm{T} 22$ & $8-13 E-25 D C C$ & $39^{\circ} 19^{\prime} 11^{\prime \prime}$ & $95^{\circ} 54^{\prime} 48^{\prime \prime}$ & 38.3 & 1.25 \\
\hline $\mathrm{T} 23$ & $8-14 E-30 B A A$ & $39^{\circ} 19^{\prime} 56^{\prime \prime}$ & $95^{\circ} 53^{\prime} 48^{\prime \prime}$ & 36.5 & 13.4 \\
\hline $\mathrm{R} 15$ & $8-13 E-36 A A A$ & $39^{\circ} 19^{\prime} 04^{\prime \prime}$ & $95^{\circ} 54^{\prime} 23^{\prime \prime}$ & 36.1 & 106 \\
\hline T24 & $8-14 E-31 B D A$ & $39^{\circ} 18^{\prime} 50^{\prime \prime}$ & $95^{\circ} 53^{\prime} 48^{\prime \prime}$ & 35.7 & 7.16 \\
\hline $\mathrm{R} 16$ & $9-13 E-12 A A A$ & $39^{\circ} 17^{\prime} 19^{\prime \prime}$ & $95^{\circ} 54^{\prime} 23^{\prime \prime}$ & 32.4 & 119 \\
\hline T25 & $9-14 E-17 A A A$ & $39^{\circ} 16^{\prime} 26^{\prime \prime}$ & $95^{\circ} 52^{\prime} 07^{\prime \prime}$ & 28.4 & 11.1 \\
\hline R17 & $9-14 E-29 B B B$ & $39^{\circ} 14^{\prime} 42^{\prime \prime}$ & $95^{\circ} 53^{\prime} 05^{\prime \prime}$ & 26.6 & 140 \\
\hline T26 & $9-14 E-29 A A A$ & $39^{\circ} 14^{\prime} 42^{\prime \prime}$ & $95^{\circ} 52^{\prime} 07^{\prime \prime}$ & 26.4 & 8.35 \\
\hline
\end{tabular}


Table 2.--List of seepage-investigation sites (concluded).

\begin{tabular}{|c|c|c|c|c|c|}
\hline $\begin{array}{l}\text { Field } \\
\text { number }\end{array}$ & $\begin{array}{c}\text { Site location } \\
\text { (station number) } \\
\end{array}$ & Lat Itude - & Longltude & $\begin{array}{l}\text { River } \\
\text { mile } \\
\end{array}$ & $\begin{array}{l}\text { Dralnage } \\
\text { area }\left(\mathrm{ml} l^{2}\right)\end{array}$ \\
\hline $\mathrm{R} 18$ & $9-1.4 \mathrm{E}-31 \mathrm{DDD}$ & $39^{\circ} 13^{\prime} 03^{\prime \prime}$ & $95^{\circ} 53^{\prime} 14^{\prime \prime}$ & 23.8 & 151 \\
\hline T27 & $10-14 E-5 B B A$ & $39^{\circ} 12^{\prime} 57^{\prime \prime}$ & $95^{\circ} 52^{\prime} 57^{\prime \prime}$ & 23.3 & 1.20 \\
\hline R19 & $\begin{array}{r}10-14 E-5 D C D \\
(06889200)\end{array}$ & $39^{\circ} 12^{\prime} 08^{\prime \prime}$ & $95^{\circ} 52^{\prime} 25^{\prime \prime}$ & 21.9 & 157 \\
\hline R20 & $10-14 E-21 D B C$ & $39^{\circ} 09^{\prime} 47^{\prime \prime}$ & $95^{\circ} 51^{\prime} 26^{\prime \prime}$ & 17.0 & 167 \\
\hline T28 & $10-14 E-21 A C D$ & $39^{\circ} 10^{\prime} 00^{\prime \prime}$ & $95^{\circ} 51 \cdot 17^{\prime \prime}$ & 16.7 & 12.0 \\
\hline R21 & $11-14 E-1 A A A$ & $39^{\circ} 07^{\prime} 43^{\prime \prime}$ & $95^{\circ} 47^{\prime} 55^{\prime \prime}$ & 11.2 & 188 \\
\hline T29 & $11-15 E-6 B B B$ & $39^{\circ} 07^{\prime} 43^{\prime \prime}$ & $95^{\circ} 47^{\prime} 47^{\prime \prime}$ & 11.0 & 74.4 \\
\hline $\mathrm{R} 22$ & $11-15 E-6 D D D$ & $39^{\circ} 06^{\prime} 57^{\prime \prime}$ & $95^{\circ} 46^{\prime} 49^{\prime \prime}$ & 9.6 & 264 \\
\hline T30 & $11-15 E-5 C D D$ & $39^{\circ} 06^{\prime} 57^{\prime \prime}$ & $95^{\circ} 46^{\prime} 16^{\prime \prime}$ & 9.1 & 9.64 \\
\hline T31 & $11-15 E-5 C D C$ & $39^{\circ} 06^{\prime} 57^{\prime \prime}$ & $95^{\circ} 46^{\prime} 24^{\prime \prime}$ & 9.1 & 9.64 \\
\hline T32 & $11-15 E-17 B B B$ & $39^{\circ} 05^{\prime} 58^{\prime \prime}$ & $95^{\circ} 46^{\prime} 41^{\prime \prime}$ & 7.9 & 8.20 \\
\hline $\mathrm{R} 23$ & $11-15 E-16 A D B$ & $39^{\circ} 05^{\prime} 45^{\prime \prime}$ & $95^{\circ} 44^{\prime} 45^{\prime \prime}$ & 7.4 & 284 \\
\hline T33 & $11-15 E-10 C B B$ & $39^{\circ} 06^{\prime} 24^{\prime \prime}$ & $95^{\circ} 44^{\prime} 28^{\prime \prime}$ & 6.8 & 5.45 \\
\hline $\mathrm{R} 24$ & $\begin{array}{c}11-15 E-14 B B C \\
(06889500)\end{array}$ & $39^{\circ} 06^{\prime} 00^{\prime \prime}$ & $95^{\circ} 43^{\prime} 27^{\prime \prime}$ & 6.0 & 290 \\
\hline T34 & $11-15 E-11 C A B$ & $39^{\circ} 06^{\prime} 24^{\prime \prime}$ & $95^{\circ} 43^{\prime} 05^{\prime \prime}$ & 5.5 & 0.57 \\
\hline R25 & $11-15 E-12 C B C$ & $39^{\circ} 06^{\prime} 17^{\prime \prime}$ & $95^{\circ} 42^{\prime} 16^{\prime \prime}$ & 4.9 & 291 \\
\hline T35 & $11-15 E-12 B D D$ & $39^{\circ} 06^{\prime} 30^{\prime \prime}$ & $95^{\circ} 41^{\prime} 51^{\prime \prime}$ & 4.5 & 22.8 \\
\hline R26 & $11-16 E-18 A D C$ & $39^{\circ} 05^{\prime} 38^{\prime \prime}$ & $95^{\circ} 40^{\prime} 19^{\prime \prime}$ & 3.0 & 316 \\
\hline T36 & $11-16 E-8 D B A$ & $39^{\circ} 06^{\prime} 24^{\prime \prime}$ & $95^{\circ} 39^{\prime} 21^{\prime \prime}$ & 1.8 & 12.3 \\
\hline R27 & $11-16 E-16 C A A$ & $39^{\circ} 05^{\prime} 32^{\prime \prime}$ & $95^{\circ} 38^{\prime} 31^{\prime \prime}$ & 1.4 & 331 \\
\hline
\end{tabular}




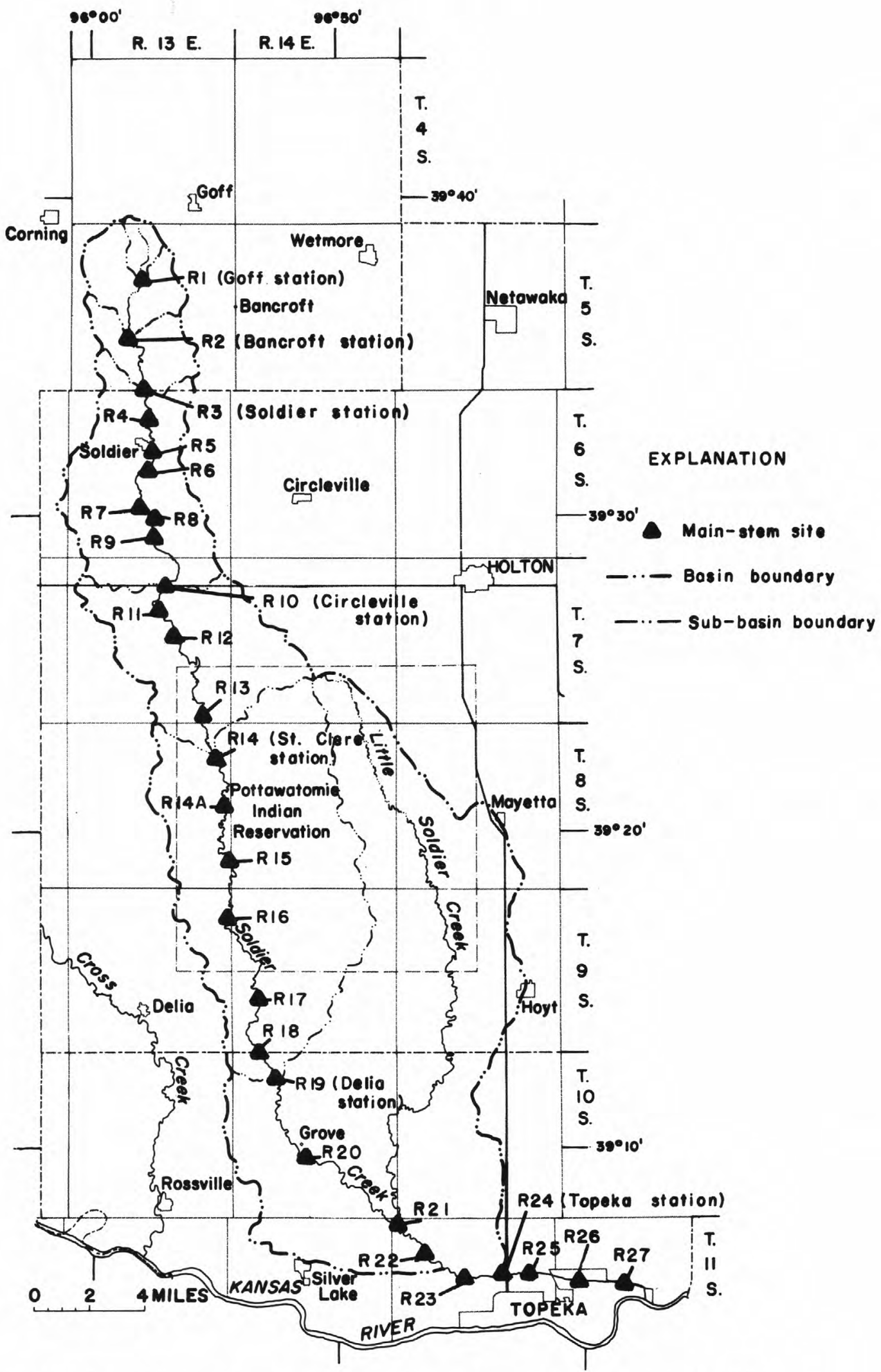

Figure 6.--Location of main-stem sites of seepage investigations. 
In August 1963, observation well s were installed to measure the water-table altitude in the alluvial deposits beneath the flood plain of Soldier Creek. At the streamflow-gaging sites near Goff, Bancroft, Soldier, Circleville, St. Clere, and Della, wells were located in a lline approximately perpendicular to the stream $(f \mathrm{~g}, 7)$. For monthly observations, holes were bored to bedrock and cased with 1.0- or 1.25-inch pipes that generally were installed with 2-foot lengths of screen. Two wells at the St. Clere site were cased with 5-inch plpe and equipped with float-operated graphic recorders for continuous measurements. The location, description, and years of avallable record at each site are given in appendix $B$.

The numbering system for well locations given in appendix $B$ is the same as that described for the rainfall-observation sites. When two or more wells are located in the same 10-acre tract, they are numbered serlally, beginning with 2, in the order in which they were installed. Altitude of land surface at each well was determined by levelling to the top of the pipe, then subtracting from that altitude a distance that would give the best approximation of the land surface at the well. Measurements of water levels in observation wells are given in appendix B with reference to LSD (land-surface datum), which is approximately the land surface at each well. Measurements are made from a fixed point that may be a short distance above or below land surface and then adjusted so that the water levels are reported in feet below LSD.

\section{DATA FOR SELECTED EVENTS OF RAINFALL AND PEAK FLOW}

This section contains a tabulation (table 3) and graphlc presentations (flgs. 8-29) of selected events of rainfall and peak flow from 1964 to 1974. The table and graphs have been included to provide a synopsis for the time and space distribution of the rainfall, peak flows, and flood hydrographs for the largest events of ralnfall and peak flow. It is hoped that presentation of the data in this form will enable the reader to determine if the data avallable for Soldier Creek basin would be useful for his studies.

Observer-rainfall data were used to obtaln the areal distribution of rainfall, which is shown with each event. The more detalled time distribution of the rainfall and resulting flood hydrograph is shown In three groups. Ralnfall and flood-hydrograph data from the Goff and Bancroft recording streamf low gages are shown together, as are data from the Soldier and Circleville recording streamflow gages. Rainfall from the St. Clere raln gage is shown in the group with the St. Clere, Della, and Topeka flood hydrographs. Rainfall is shown in inches, and discharge on the flood hydrograph is shown in cublc feet per second. Both time and flood hydrograph scales may differ from one group to the next. 

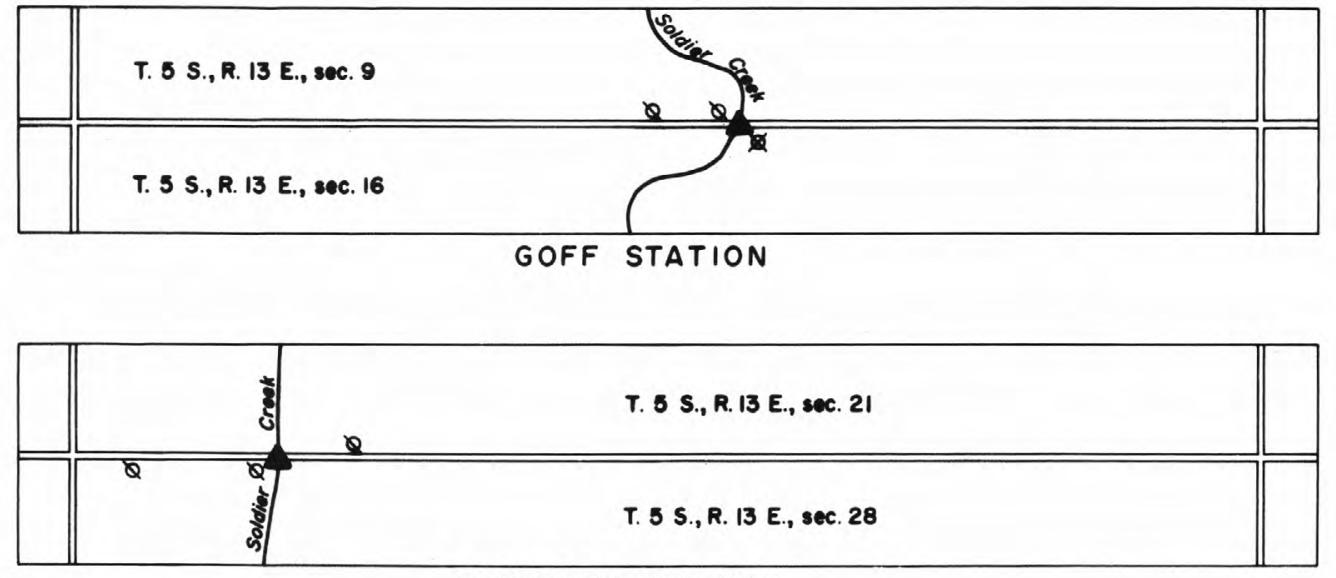

BANCROFT STATION

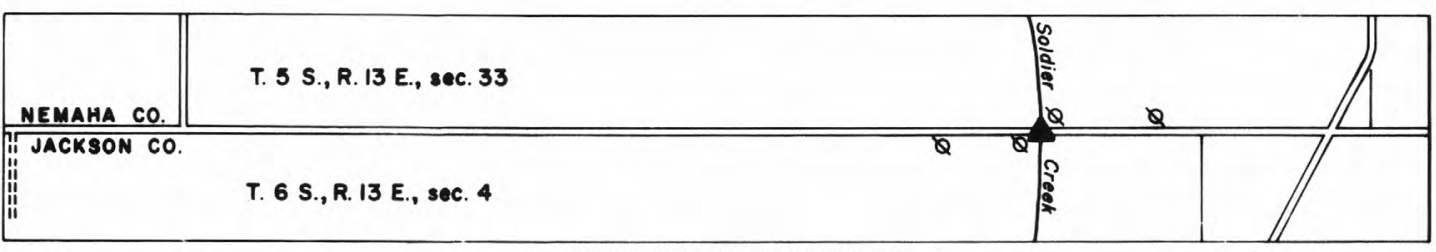

SOLDIER STATION

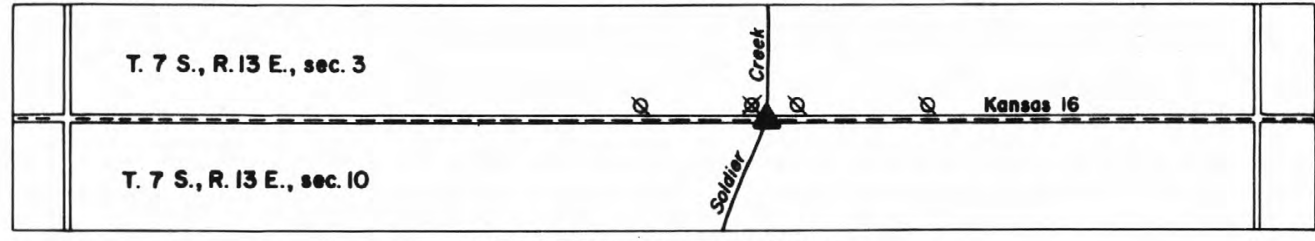

CIRCLEVILLE STATION

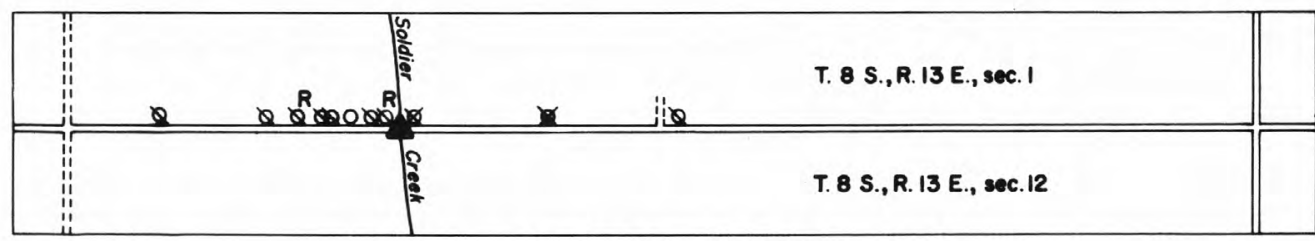

ST. CLERE STATION
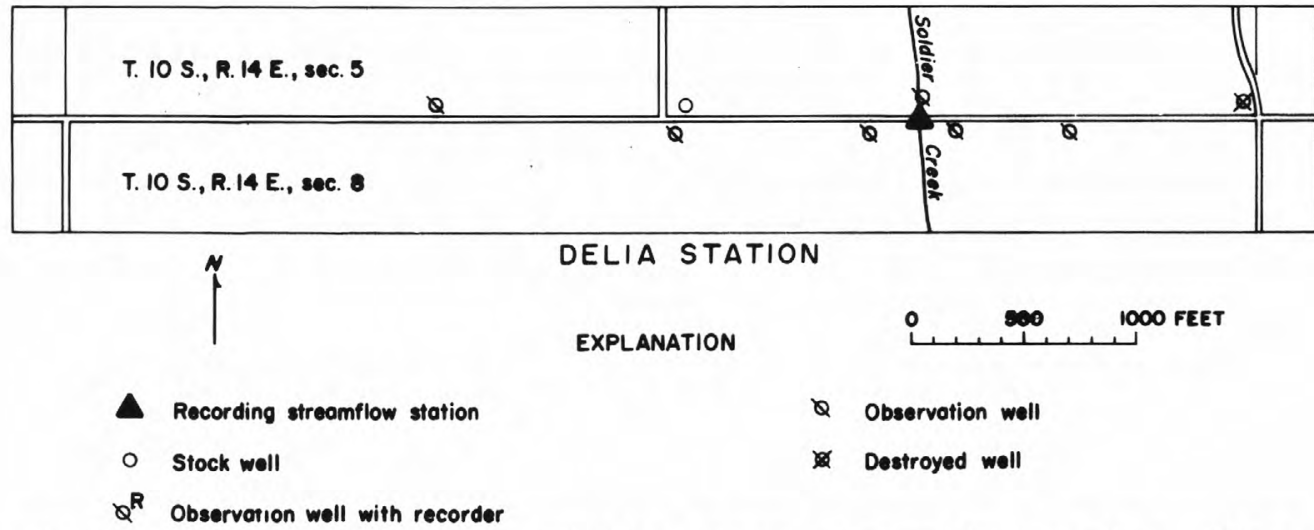

EXPLANATION

Q Observation well

- Destroyed woll

$Q^{R}$ Observation well with recorder

Figure 7.--Location of water wells at the upper streamflow-gaging sites. 
1964 WATER YEAR

Goff
Soldier

Daily Peak Daily Peak Dally Peak

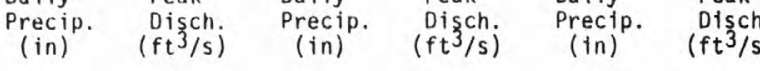

$4-22-64$

$4-23-64$

$4-24-64$

$4-25-64$

$4-26-6$

$4-27-64$

0.82

0

.14

1.98
.04

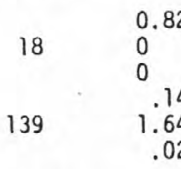

0.82

.14

$5-01-64 \quad .50$

$7.3 \quad 0^{.52}$

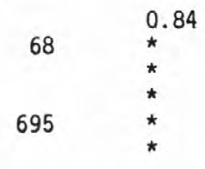

84

960

$24 \quad 0^{.62}$

.36

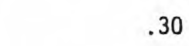

$5-06-64 \quad .34$

5-24-64 .28

$5-25-64$

$5-26-64$

$5-28-64$

$5-29-64$

$\begin{array}{ll}0-04-64 & 2.14 \\ 6-05-64 & 0\end{array}$

$6-11-64$

\subsection{0}

$6-12-64$

$6-13-64$

$6-14-64$

.86

0
1.30

$6-15-64 \quad 0$

$6-21-64$

$\begin{array}{ll}6-22-64 & .92 \\ 6-23-64 & .02\end{array}$

7-08-64 . 14

$7-11-64 \quad .18$

$8-27-64 \quad 1.18$

$8-28-64 \quad 0$

$8-31-64 \quad 1.04$

10.30

$\begin{array}{ll}1.6 & .72 \\ 2.8 & .74 \\ & .02\end{array}$

$0^{.02}$

$<1.54$

$\begin{array}{rr}<1 & 1.00 \\ 1.0 \quad .72 \\ \end{array} \quad .02$

$0^{.02}$

$\begin{array}{ll}26 & 2.84 \\ & 0\end{array}$

64
112

$\begin{array}{ll}112 & 0.94 \\ 239 & 1.00\end{array}$

1.44
.94
0.00
1.00
.02

$\begin{array}{ll}390 & 2.82 \\ & 0\end{array}$

$\begin{array}{ll}420 & 1.20\end{array}$

$669 \quad .96$

$\begin{array}{ll} & 0 \\ & \\ 982 & 1.12\end{array}$

$770 \quad \begin{aligned} & 1.22 \\ & 1.54\end{aligned}$

1.48
$192 \quad 1.34$
.02

.18

.14

.58

1.16

.24

$\begin{array}{lr}11-03-64 & 1.00 \\ 11-04-64 & .02\end{array}$

$11-15-64 \quad 1.60$

$11-16-64 \quad .08$

$\begin{array}{ll}1-01-65 & 0.74\end{array}$

$\begin{array}{ll}2-28-65 & 1.42\end{array}$

$3-01-65$

$$
.12
$$

.80

$3-16-65$
$3-17-65$

.22

$4-24-65$

:

$5-18-65 \quad .52$

$5-19-65 \quad 0^{.52}$

$5-21-65 \quad .48$

$\begin{array}{lll}.48<1 & <8\end{array}$

$5-24-65 \quad .50$

$\begin{array}{ll}5-25-65 & .18 \\ 5-26-65 & .06\end{array}$

$\begin{array}{ll}5-31-65 & .18 \\ 6-01-65 & .44 \\ 6-02-65 & 0\end{array}$

$6-04-65 \quad .68$

$6-05-65$

$6-06-65$

$6-07-65$

$6-08-65$

$6-09-65$

$6-10-65$

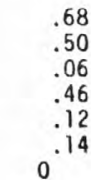

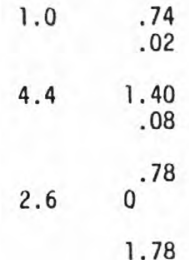

1.78
$187 \quad .14$

$\begin{array}{rr} & .72 \\ 34 \quad .24\end{array}$

.50

$0^{.42}$

.42
.20

.06

.20
.52

$0^{.52}$

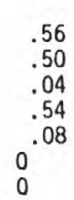

$3 \quad .56$

3
18
.14
.98
.02

$\begin{array}{cc}1 & 1.10 \\ 2.3 & 0 \\ 15 & .82 \\ & .80 \\ & 0\end{array}$

$1340 \quad 2.28$
.04

$\begin{array}{lll} & .76 \\ 1540 \quad & .80\end{array}$

$\begin{array}{ll}1540 & 0 \\ & 1.08 \\ 1150 & 0\end{array}$

$1430 \quad \begin{aligned} & .74 \\ & 1.96\end{aligned}$

1965 WATER YEAR

1.42
.10

$0^{.94}$

$\begin{array}{rl}15 & 1.54 \\ 6 & 0\end{array}$

$1510 \quad .98$

1.76

.12

.76
.20

.54

$0^{.74}$

.50

.50

.22

. .42

I/ NWS - Rainfall records at Topeka from National Weather Service

*Gage Malfunction

Note: Rainfall data for Circleville is questionable June 7-9, 1965.
Circleville

Daily Peak

Disch
$\left(\mathrm{ft}^{3} / \mathrm{s}\right)$

$$
\text { (in) }
$$

0.86
0
0
.14

105

1270

$0^{.52}$

.32

1

174

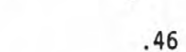

$1.1 \quad .96$

$2.100^{.80}$

$1.6 \quad 0.80$

\section{$0.3 \quad 1.18$}

.04

$\begin{array}{ll}1.3 & 1.52 \\ 0 & .02\end{array}$

.74
$\star \quad .16$

.58
.24

St. Clere Delia Topeka Topeka NWS

Dally Peak Peak Peak Dafly

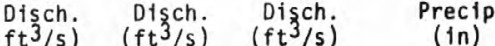

(in) $\left(\mathrm{ft}^{3} / \mathrm{s}\right)\left(\mathrm{ft}^{3} / \mathrm{s}\right) \quad\left(\mathrm{ft}^{3} / \mathrm{s}\right)$

$\begin{array}{rrrrr}0.86 & & & 127 & 0.81 \\ .20 & 180 & 91 & & 0 \\ .04 & & & & . .54 \\ .08 & 1150 & 903 & 670 & \mathrm{~T}\end{array}$

$.52 \quad 110$

$.84 \quad 95$

$.92 \quad 92$

.56

.64
.06

130

$1.00 \quad 2890$

$3100 \quad \begin{array}{r}1.00 \\ .10\end{array}$

1.26

.98

$\begin{array}{ll} & 0 \\ 2300 & 1.18 \\ & 0\end{array}$

3010

2350

.84
1.52

6310

.80

21

2.72

614

7.5

$0^{.52}$

14

1.04

1.10

21

2.26

92

$814 \quad 1560$

4.66
.15

$420 \quad 1.62$

897

2170

$\begin{array}{lll} & .62 & \\ 4160 \quad .10 & 3890\end{array}$

$\begin{array}{lll}2480 & 2640 & .20 \\ .34\end{array}$

$598 \quad .7$

$882 \quad 1920$

106

$538 \quad 1130$

83

318

$244 \quad 313$

$16 \quad .76$

16

.62

19

31

112

1140

$40 \quad 0^{.336}$

414

. .46

345

228

268

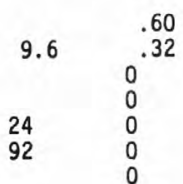

$\begin{array}{rr}93 & .58 \\ & 0.36 \\ 182 & .50 \\ & .06 \\ & 0.66\end{array}$

131

221

124

152

$\begin{array}{ll}846 & 1.37 \\ & 0.54 \\ 217 & 0.57 \\ 685 & 1.07 \\ & 0\end{array}$

$\mathrm{T}^{.06}$

$T^{.98}$

.81
.03
.34
53

.53

$0^{.11}$

.24

$0^{.19}$

$i^{.03}{ }^{.03}$

.64

.23
1.80

.50

.01

.04
1.25

.63

.45

$\mathrm{T}^{.64}$

3.28

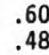

$0^{.67}$

.88
.04

.34

06

.53

$T .99$
.98

.38
.48

1. 37

.54

.29
1.07 
1965 WATER YEAR--Continued

Gof

Bancroft

Soldier

Circleville

St. Clere

Delia

Topeka Topeka NWS 1 !

Date Daily Peak Daily Peak Dally Peak Peak Daily Peak Daily Peak

Peak

Peak

Daily

(in) $\left(\mathrm{ft}^{3 / \mathrm{s}}\right) \quad$ (in) $\left(\mathrm{ft}^{3} / \mathrm{s}\right)$

$\begin{array}{lr}6-25-65 & 1.86 \\ 6-26-65 & 1.16 \\ 6-27-65 & 1.50 \\ 6-28-65 & .44 \\ 6-29-65 & .14 \\ 6-30-65 & .16 \\ 7-01-65 & .28 \\ 7-02-65 & .48\end{array}$

$177 \quad 1.40$

\begin{tabular}{rr}
70 & 1.30 \\
238 & 1.28 \\
6 & .66 \\
19 & .14 \\
19 & .14 \\
\hline
\end{tabular}

$\begin{array}{lr}444 & .24 \\ 588 & 2.58\end{array}$

$\begin{array}{rr}588 & 2.58 \\ 1360 & 1.42\end{array}$

25

10
82

82
283

.18
.16
.34

.30

$\begin{array}{ccc}\text { Disch. } & \text { Precip. } & \text { Disch } \\ \left(\mathrm{ft}^{3} / \mathrm{s}\right. & \text { (in) } & \left(\mathrm{ft}^{3} / \mathrm{s}\right.\end{array}$

(in)

450

$\begin{array}{lllll}450 & 0 & & .12 & \\ 656 & 2.00 & 1210 & 1.56 & 1170\end{array}$

1580

31

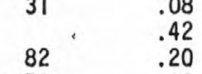

678

2.00
1.32
.54
.08
.42
.20
.04
1.64

1210

$\begin{array}{llll}\left(\mathrm{ft}^{3} / \mathrm{s}\right) & \left(\mathrm{ft}^{3} / \mathrm{s}\right) & \left(\mathrm{ft}^{3} / \mathrm{s}\right) & \text { (in) }\end{array}$

1170

$\begin{array}{rr}3640 & .76 \\ 191 & .10 \\ 1070 & .82\end{array}$

3980

1.10

528
706

$7-06-65$

$0^{.04}$

103

$7-07-65$

18 *

$7-11-65$
$7-12-65$$\quad 0^{.38}$

49 *

336

$7-27-65 \quad .40$

$0^{.38}$

$7-28-65$

0

$8-17-65 \quad .46$

.48

.02

119

0

367

$0^{.50}$

1460

$0^{.50}$

345

$0^{.20}$

$0^{.74}$

1.68

$0^{.46}$

.68

8-24-65 . 16

.20
.22

$9-03-65 \quad .86$

1.04
1.80

$9-04-65 \quad 1.66$

$\begin{array}{ll}9-09-65 & 1.14 \\ 9-10-65 & .10 \\ 9-11-65 & 0\end{array}$

1.12

9-18-65 $9-19-65$

$9-20-65$

$9-21-65$
$9-22-65$

$\begin{array}{lll}.54 & 1.1 & .58 \\ .46 & & .32 \\ 2.74 & 368 & 2.90 \\ 0 & & 0 \\ 0 & & \end{array}$

$0^{.16}$

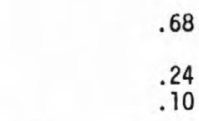

2

1.14

.36
.04

$\begin{array}{lr}<1 & .44 \\ 90 & 2.72\end{array}$

27

1.52
0
0

$\begin{array}{rr}3.6 & .56 \\ & .30 \\ 2140 & 2.68 \\ & 0.26\end{array}$

.56
.30
2.68
.26

13
2330

2.36

.94
.20

.72
.30
3.08
.02

1966 WATER YEAR

$\begin{array}{llll}12-23-65 & 1.14 & 14 & 1.20\end{array}$

$\begin{array}{ll}12-24-65 & .08\end{array}$

$\begin{array}{lll}1-01-66 & .22 & .26\end{array}$

$1-02-66 \quad .06$

$61 \quad 1.18$
$6 \quad .08$

1.12
.08

446

1.14
.12

434

935

$0^{.50}$

825

$0^{.06}$

$5-10-66$
$5-11-66$

$5-12-66$

.24

$6-07-66$

.18
1.54
0

$26 \quad \begin{aligned} & .20 \\ & 0\end{aligned}$

6-09-66

$6-12-66 \quad 44$

$\begin{array}{ll}6-12-66 & .44 \\ 6-13-66 & .02\end{array}$

6-26-66 .64

$\begin{array}{lll}2.00 & 3 & 1.00\end{array}$

$7-07-66$

$7-25-66$

$7-26-66$

.44

.54

$8-09-66$

$8-10-66$

$8-20-66$

$8-21-66$

8-22-66

$9-01-66$

$9-02-66$

9-03-66

$9-04-66$

.74

1.20

$0^{.42}$

2.08

0.08
1.00

1.00

$9-17-66$

$9-18-66$

1.16

1.16
0
0

$9-19-66$
$0^{.56}$

.56

.98

.10
.08

.76

.76
.08

2

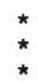

$83 \quad \begin{aligned} & 1.86 \\ & 0.06\end{aligned}$

.46
.32

$78 \quad 1.28$
$\quad 1.36$

$36 \quad .50$
$\quad .04$

.30

$44 \quad .90$

1.56

.04
.10

.82

.42

$\begin{array}{ll} & .88 \\ 6 & .44 \\ & .20\end{array}$

408

1.16
.08
.26
0

$<\quad 1.04$

$0^{.02}$

13

$0^{.02}$
$110-1.16$
846

468

$0^{.70}$

$0^{.08}$

.44
.28

$77 \quad .20$
$\quad 1.82$

$23 \quad .50$
$\quad .06$

.28

.86

273

.48
.02
.10

1.14

1.14
.46

1.40
.02

$0^{.02}$

1.16
.02
.14
.02

$304 \quad \begin{array}{ll}.16 \\ 2.22\end{array}$

2170

.10
.10

.22

.70

321

.08
.08
.10

.90
.88

2.02
.70

72

315

$10 \quad 0^{.06}$
108

238

$120_{0}^{.14}$
149

119
443

1380

49

49

700

$.76 \quad 63$

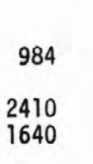

1680

.75

.52
1.58

6940

3810

1830

T.74

.14

1.19

111

(1)

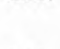

125

.72

.06

$\mathrm{T}^{.32}$

$913 \quad 1110 \quad \begin{aligned} & .14 \\ & 1.44\end{aligned}$

504

493

.94
.26

160

5380

5950

.72
.74
2.09
.05
0

1.32

.09

.22

90

427

$0^{.08}$

1400

1440

1.37

$84 \quad 183$

2140

$\begin{array}{r}.53 \\ \hline\end{array}$

1780

1.65

20
48

.24

T.18

.69
.58

218

35
60

35
60

49

76 
Goff

Bancroft

Soldier

circleville

St. Clere

Della Topeka Topeka NWS $1 /$

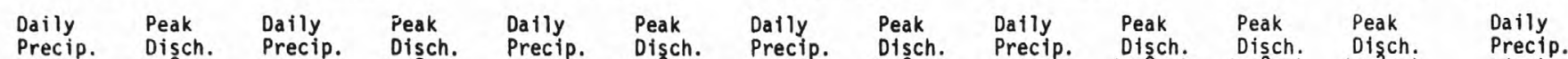

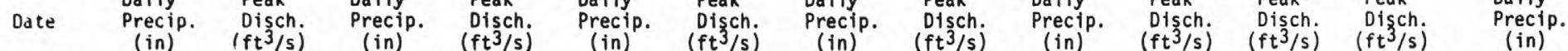

$\begin{array}{ll}3-31-67 & .62 \\ 4-01-67 & 1.28 \\ 4-02-67 & 0 \\ 4-03-67 & .02\end{array}$

$16 \quad \frac{1.54}{0} \quad \frac{.24}{16}$

$68 \quad \begin{aligned} & . .62 \\ & \quad 0^{1.44}\end{aligned}$

$135 \quad \frac{1.52}{0} \quad 0^{1.32}$

$501 \quad 1.34$
$\quad .06$

501

457

$\begin{array}{rr} & 1.05 \\ & 2.95 \\ 511 \quad .66 \\ 1.26\end{array}$

4-12-67 $\quad .10$

$\begin{array}{ll}4-12-67 & 1.10 \\ 4-13-67 & 1.24 \\ 4-14-67 & 0\end{array}$

$28 \quad \begin{aligned} & .12 \\ & 0\end{aligned}$

$\begin{array}{llll}136 & 1.14 & & .12 \\ 0 & 314 & 1.62\end{array}$

$1270 \quad \int_{0}^{. .22} \quad 1230$

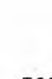

51

$\begin{array}{rr}5-29-67 & .62 \\ 5-30-67 & .14 \\ 5-31-67 & 1.44\end{array}$

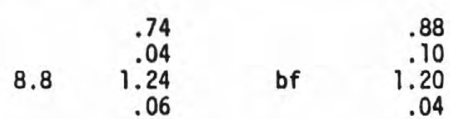

.92

$\quad .92$
$220 \quad 1.22$
.02

$\begin{array}{rrr} & 1.40 & \\ 1440 \quad 1.14 & 49 \\ 1.16 & 1570\end{array}$

795

792

.57
1.79

$0-01-67 \quad .06$

.46

$710 \quad \quad .14$

636

.14

$\begin{array}{lr}2880 \quad 28 & .28 \\ & 2.52\end{array}$

$\begin{array}{rr}6-05-67 & 1.48 \\ 6-06-67 & .02\end{array}$

$162 \quad .68$

2.18

2.

6-09-67 1.68

$6-10-67$

$\begin{array}{llll}222 & 1.68 & 932 & 1.7 \\ 27 & .60 & 238 & .50 \\ 133 & \star & & 1.66 \\ 198 & \star & 800 & \end{array}$

$1.72 \quad 900$

$900 \quad 2.76 \quad 3110$
301

$\begin{array}{ll}1.68 & 2930 \\ 1.84 & 6520\end{array}$

$6-11-67 \quad *$

$6-12-67$

1.66

$1070 \quad \begin{aligned} & 1.06 \\ & 0\end{aligned}$

3820

5430

6-19-67 .26

$\begin{array}{lr}6-20-67 & .18 \\ 6-21-67 & 1.08\end{array}$

$\begin{array}{lr}6-21-67 & .08 \\ 6-22-67 & .02\end{array}$

$6-23-67 \quad 0.02$

$\begin{array}{ll}6-24-67 \\ 6-25-67 & 0.78\end{array}$

149

.22

.58
.26
.36

.20
1.48

$0^{.02}$

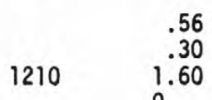

1210

534 * 486

$46 \quad .68$

227

0.04

$322 \quad \begin{aligned} & .04 \\ & 1.24\end{aligned}$

2970

$\begin{array}{ll}7-08-67 & 1.48\end{array}$

21

.66
1.16
.04

162

1.54
.02

461

1.02
0
0

197

3110

1080

1210

1.06

.29
1.35

$7-10-67 \quad 0$

$\begin{array}{ll}9-19-67 & .70 \\ 9-20-67 & .54 \\ 9-21-67 & 0\end{array}$

$0^{.62}$

.48
0

$\begin{array}{ll}2 & .64 \\ 4 & 1.52\end{array}$

1968 WATER YEAR

10-05-67

$\begin{array}{rr}10-06-67 & 1.76 \\ 10-07-67 & .48\end{array}$

.68
1.56
.68

.38
1.56

$5-13-68$

1.54

32

1.30

225

.62

236

236

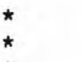

1.54
.02

$0^{1.30}$

105

$0^{.96}$

160

1190

*

85

2200

1390

3920

3410

.10

5-15-68

2.26

87

1.38

176

.64

$7-16-68$
$7-17-68$

3.14

279

2.94

860

$7-23-68$
$7-24-68$

$7-25-68$

7-26-68

$0^{.58}$

$7-27-68$

$0^{.14}$

42
34

2.94
.68
.02
.90

$\begin{array}{ll}860 & 2.54 \\ & 1.02 \\ & 0 \\ 265 & 1.88 \\ 213 & .08\end{array}$

151

106

7080

7580

18600

284
356

2780

18300

2240

3420

323

224

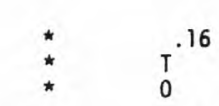

$7-30-68$
$7-31-68$

.70
$.40 \quad 117$

$8-01-68$

$8-02-68$

.26

314

373

.92
0.10

610

.52
.38

179

*

106

8
26

184

22

29

122

.69
1.69

8-03-68

.02

1730

.54

578
780

2090

940
1380

836

1010

2100
2440 3430

2440
2280

.61

1.90

$8-08-68$

$8-09-68$

.44
$.98 \quad 15$
.98

$8-10-68$

75
152

1.32

$339 \quad .30$
547 $\quad 1.46$

470
85

1350
3120

$1350 \quad$ * $\quad 1790$

3310

1540

2310

3400

3580

3490

6350

$0^{.12}$

9-03-68

$9-04-68$

120

2.06

438

1.92

1520

1480
3100

$9-23-68$

81

1.18

358

$0^{.94}$

456

1969 WATER YEAR

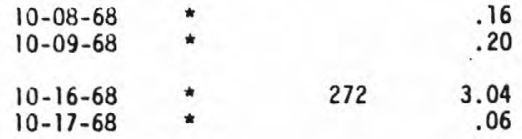

.10
.22

1320

.10
.22

1460

*

845 *
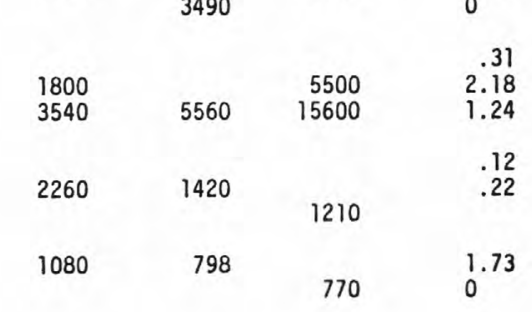

of Indicates base flow. 


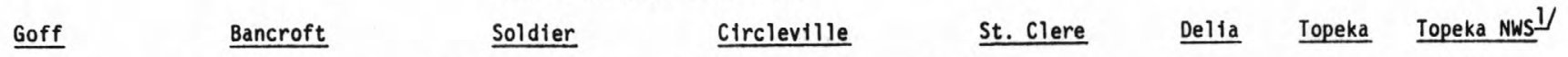

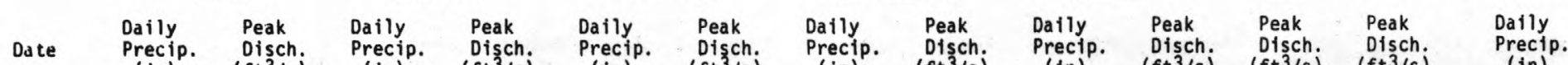

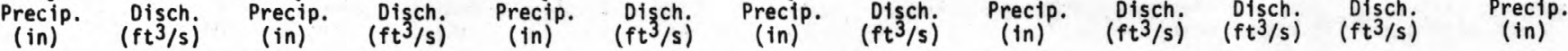

\begin{tabular}{|c|c|c|c|c|c|c|c|c|c|c|c|c|c|}
\hline $\begin{array}{l}3-22-69 \\
3-23-69 \\
3-24-69\end{array}$ & " & 70 & $\begin{array}{r}.60 \\
1.08 \\
.34\end{array}$ & 491 & $\begin{array}{r}.60 \\
1.02 \\
.30\end{array}$ & 586 & $\begin{array}{l}0 \\
1.12 \\
.26\end{array}$ & 1570 & $\begin{array}{l}* \\
* \\
*\end{array}$ & 2170 & 2020 & 2250 & $\begin{array}{l}.75 \\
.24\end{array}$ \\
\hline $\begin{array}{l}4-03-69 \\
4-04-69 \\
4-05-69\end{array}$ & $\begin{array}{l}1.14 \\
0^{.82}\end{array}$ & 384 & $0^{.80}$ & 1260 & $0^{.30}$ & 1240 & $0^{.26}$ & 1860 & $0_{0}^{.32}$ & 2070 & 2030 & 2860 & $\begin{array}{l}.03 \\
1.94 \\
T\end{array}$ \\
\hline $\begin{array}{l}4-16-68 \\
4-17-69\end{array}$ & $\begin{array}{r}1.38 \\
.08\end{array}$ & 95 & $\begin{array}{r}1.56 \\
.08\end{array}$ & 465 & $\begin{array}{r}1.96 \\
.14\end{array}$ & 474 & $\begin{array}{r}1.18 \\
.14\end{array}$ & 1380 & $\begin{array}{l}1.32 \\
0\end{array}$ & 1570 & 1520 & 1790 & $\begin{array}{r}1.82 \\
.04\end{array}$ \\
\hline $\begin{array}{l}4-25-69 \\
4-26-69 \\
4-27-69\end{array}$ & 2.02 & 233 & $\begin{array}{l}0 \\
2.24 \\
0\end{array}$ & 1200 & $\begin{array}{l}0 \\
2.31 \\
0\end{array}$ & 1420 & $\begin{array}{l}* \\
* \\
*\end{array}$ & 3000 & $\begin{array}{l}0 \\
2.82 \\
0\end{array}$ & 7140 & 4450 & 7310 & $\begin{array}{l}0 \\
3.24 \\
0\end{array}$ \\
\hline $\begin{array}{l}5-07-69 \\
5-08-69\end{array}$ & $\begin{array}{l}1.87 \\
0\end{array}$ & 211 & $0^{1.71}$ & 954 & $\begin{array}{r}2.07 \\
.02\end{array}$ & 905 & $\begin{array}{r}1.49 \\
.01\end{array}$ & 2400 & $\begin{array}{l}.82 \\
.01\end{array}$ & 3240 & 2360 & 2250 & $\begin{array}{r}1.55 \\
.05\end{array}$ \\
\hline $\begin{array}{l}5-13-69 \\
5-14-69 \\
5-15-69 \\
5-16-69 \\
5-17-69\end{array}$ & $\begin{array}{l}. .42 \\
.09 \\
.79\end{array}$ & $\begin{array}{r}1.5 \\
4 \\
40\end{array}$ & $\begin{array}{l}0.47 \\
.09 \\
1.05 \\
0\end{array}$ & 194 & $\begin{array}{l}0.55 \\
.12 \\
.96 \\
0\end{array}$ & 370 & $\begin{array}{l}\text { * } \\
\text { * } \\
\text { * } \\
\text { * }\end{array}$ & $\begin{array}{r}276 \\
\\
150 \\
1980\end{array}$ & $\begin{array}{l}. .68 \\
.19 \\
.48\end{array}$ & 1960 & $\begin{array}{c}361 \\
1440\end{array}$ & $\begin{array}{r}432 \\
1550\end{array}$ & $\begin{array}{l}.31 \\
.27 \\
. \\
T^{.11}\end{array}$ \\
\hline $\begin{array}{l}5-21-69 \\
5-22-69\end{array}$ & $0^{.86}$ & 125 & $0^{.95}$ & 602 & * & 690 & $\stackrel{*}{*}$ & 2300 & $\begin{array}{r}1.53 \\
.01\end{array}$ & 2960 & 2600 & 2630 & $\begin{array}{r}1.06 \\
.01\end{array}$ \\
\hline $\begin{array}{l}6-11-69 \\
6-12-69 \\
6-13-69\end{array}$ & $\begin{array}{l}1.80 \\
.08\end{array}$ & 22 & $\begin{array}{l}1.82 \\
. .10\end{array}$ & 210 & $\begin{array}{l}1.82 \\
.21\end{array}$ & 458 & $\stackrel{*}{*}$ & 1010 & $\begin{array}{l}1.54 \\
0.13\end{array}$ & 875 & 778 & 816 & $\begin{array}{l}2.72 \\
.01\end{array}$ \\
\hline $\begin{array}{l}6-17-69 \\
6-18-69\end{array}$ & $0^{1.26}$ & 15 & $\begin{array}{l}1.50 \\
0\end{array}$ & 148 & $\begin{array}{l}1.73 \\
0\end{array}$ & 282 & * & 3240 & $0^{1.72}$ & 3760 & 2620 & 2660 & ${ }_{0}^{1.39}$ \\
\hline $\begin{array}{l}6-21-69 \\
6-22-69\end{array}$ & $\begin{array}{r}.03 \\
1.73\end{array}$ & 83 & $\begin{array}{r}.03 \\
1.22\end{array}$ & 417 & $\begin{array}{l}.06 \\
1.24\end{array}$ & 484 & * & 3060 & 1.21 & 3110 & 2690 & 3270 & $\begin{array}{r}.94 \\
1.02\end{array}$ \\
\hline $\begin{array}{l}7-07-69 \\
7-08-69 \\
7-09-69 \\
7-10-69\end{array}$ & $\begin{array}{l}.19 \\
.02 \\
1.18 \\
0\end{array}$ & 240 & $\begin{array}{l}\star 13 \\
\star \\
\star\end{array}$ & 1200 & $\begin{array}{l}.09 \\
.03 \\
.48 \\
.03\end{array}$ & 2350 & $\begin{array}{l}* \\
* \\
* \\
*\end{array}$ & 3080 & $\begin{array}{l}* \\
* \\
* \\
*\end{array}$ & 2940 & 1760 & 2300 & $\begin{array}{r}.45 \\
.73 \\
.11\end{array}$ \\
\hline $8-20-69$ & 1.64 & 6.4 & 1.95 & 19 & * & * & * & & .32 & & & & $\mathrm{~T}$ \\
\hline $9-05-69$ & .47 & & .67 & 1.1 & * & 4.4 & * & & .92 & 20 & & & 1.18 \\
\hline & & & & & & 1970 WAT & YEAR & & & & & & \\
\hline $10-05-69$ & 1.04 & .3 & 1.31 & .8 & 1.72 & 15 & .87 & 16 & 1.20 & 12 & & & .57 \\
\hline $\begin{array}{l}10-09-69 \\
10-10-69\end{array}$ & $\begin{array}{r}1.04 \\
.52\end{array}$ & 45 & $\begin{array}{l}.53 \\
.41\end{array}$ & & $\begin{array}{l}.15 \\
.59\end{array}$ & & $\star^{.02}$ & & $\begin{array}{l}.04 \\
.69\end{array}$ & & & & $\begin{array}{r}.27 \\
1.04\end{array}$ \\
\hline $\begin{array}{l}4-18-70 \\
4-19-70\end{array}$ & $\begin{array}{l}1.39 \\
0\end{array}$ & 21 & $0^{.70}$ & 65 & $0^{1.00}$ & 160 & $0^{.61}$ & 525 & $\begin{array}{l}1.28 \\
0\end{array}$ & 595 & 539 & 655 & $\begin{array}{l}2.10 \\
0\end{array}$ \\
\hline $\begin{array}{l}4-30-70 \\
5-01-70\end{array}$ & .81 & 1 & $\begin{array}{l}.30 \\
.01\end{array}$ & & $0^{.82}$ & 57 & $0^{.53}$ & & $\begin{array}{l}1.14 \\
0\end{array}$ & 460 & 380 & 998 & $T^{.52}$ \\
\hline $\begin{array}{l}5-09-70 \\
5-10-70 \\
5-11-70\end{array}$ & $\begin{array}{r}1.52 \\
4.02 \\
.01\end{array}$ & 7080 & $\begin{array}{l}.41 \\
1.65 \\
0\end{array}$ & $13100^{*}$ & $\begin{array}{l}1.22 \\
1.48 \\
0\end{array}$ & 11700 & $\begin{array}{l}. .40 \\
. .25\end{array}$ & $\begin{array}{r}483 \\
5570\end{array}$ & $0^{.61}$ & $\begin{array}{r}603 \\
7830\end{array}$ & $\begin{array}{r}438 \\
2610\end{array}$ & $\begin{array}{r}456 \\
2270\end{array}$ & $\tau^{.07}$ \\
\hline $\begin{array}{l}5-13-70 \\
5-14-70\end{array}$ & $\begin{array}{l}.11 \\
.75\end{array}$ & 204 & ${ }^{0} .18$ & 509 & $\begin{array}{l}.03 \\
.34\end{array}$ & 537 & $\begin{array}{l}.04 \\
.28\end{array}$ & 675 & $\begin{array}{l}.01 \\
.56\end{array}$ & 714 & 662 & 958 & $\begin{array}{l}.62 \\
.96\end{array}$ \\
\hline $\begin{array}{l}5-22-70 \\
5-23-70 \\
5-24-70 \\
5-25-70\end{array}$ & $\begin{array}{l}0 \\
1.12 \\
.63 \\
.30\end{array}$ & $\begin{array}{r}126 \\
36\end{array}$ & $\begin{array}{l}0 \\
.23 \\
.36 \\
.05\end{array}$ & $\begin{array}{l}331 \\
266\end{array}$ & $\begin{array}{l}0 \\
0 \\
.52 \\
.32\end{array}$ & $\begin{array}{l}306 \\
266\end{array}$ & $\begin{array}{l}0 \\
.26 \\
.32 \\
.03\end{array}$ & $\begin{array}{l}211 \\
259\end{array}$ & $\begin{array}{r}.06 \\
1.01 \\
.21 \\
.40\end{array}$ & $\begin{array}{l}254 \\
436\end{array}$ & 1100 & 1660 & $\begin{array}{l}0 \\
.03 \\
.14 \\
.92\end{array}$ \\
\hline $\begin{array}{l}5-28-70 \\
5-29-70\end{array}$ & $\begin{array}{l}1.00 \\
0\end{array}$ & 104 & $0^{.27}$ & 190 & $0^{.76}$ & 167 & $0^{.30}$ & & $\begin{array}{l}.51 \\
.08\end{array}$ & 216 & 370 & 1110 & .83 \\
\hline $\begin{array}{l}5-31-70 \\
6-01-70 \\
6-02-70 \\
6-03-70 \\
6-04-70\end{array}$ & $\begin{array}{r}1.02 \\
.10 \\
.70 \\
1.07 \\
.02\end{array}$ & 126 & $\begin{array}{l}.36 \\
.04 \\
.22 \\
.32 \\
.02\end{array}$ & $\begin{array}{r}350 \\
558\end{array}$ & $\begin{array}{l}.72 \\
.09 \\
.57 \\
.80 \\
.02\end{array}$ & $\begin{array}{l}350 \\
585\end{array}$ & $\begin{array}{l}.62 \\
.09 \\
.23 \\
.26 \\
0^{26}\end{array}$ & $\begin{array}{r}710 \\
1470\end{array}$ & $\begin{array}{l}1.22 \\
.05 \\
.86 \\
.91 \\
.02\end{array}$ & $\begin{array}{l}2240 \\
2450\end{array}$ & $\begin{array}{l}1550 \\
3930\end{array}$ & 2300 & $\begin{array}{r}.86 \\
.43 \\
1.15 \\
.73 \\
.05\end{array}$ \\
\hline $\begin{array}{l}6-11-70 \\
6-12-70\end{array}$ & $0^{.73}$ & & $\begin{array}{l}.15 \\
.01\end{array}$ & & $0^{.36}$ & & $0^{.31}$ & & $0^{.69}$ & 85 & 968 & 2580 & ${ }_{\mathrm{T}}^{2.09}$ \\
\hline $\begin{array}{l}9-16-70 \\
9-17-70\end{array}$ & $\begin{array}{r}1.17 \\
.22\end{array}$ & * & $\begin{array}{l}.19 \\
.16\end{array}$ & & $\begin{array}{l}.30 \\
.27\end{array}$ & & $\begin{array}{l}.50 \\
.18\end{array}$ & & $\begin{array}{r}.20 \\
1.01\end{array}$ & & 812 & 1420 & $\begin{array}{l}.35 \\
.28\end{array}$ \\
\hline
\end{tabular}


Goff Bancroft $\underline{\text { Soldier }}$ Circleville st.clere Delia Topeka $\underline{\text { Topeka NWS }} 1 /$

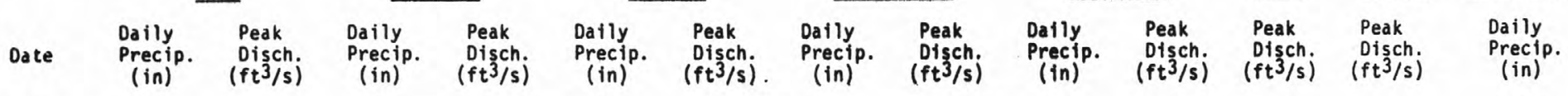

\begin{tabular}{|c|c|c|c|c|c|c|c|c|c|c|c|c|}
\hline $\begin{array}{l}9-21-70 \\
9-22-70 \\
9-23-70 \\
9-24-70\end{array}$ & $\begin{array}{l}.56 \\
1.29 \\
. .69\end{array}$ & $\begin{array}{l}.20 \\
* \quad .23 \\
.39\end{array}$ & $\begin{array}{l}322 \\
114\end{array}$ & $\begin{array}{l}.70 \\
1.16 \\
.62 \\
0\end{array}$ & 350 & $\begin{array}{l}1.01 \\
.34 \\
.38 \\
0\end{array}$ & 1070 & $\begin{array}{l}1.37 \\
.61 \\
0.15\end{array}$ & $\begin{array}{l}1080 \\
1050\end{array}$ & $\begin{array}{l}1820 \\
1720\end{array}$ & $\begin{array}{l}4270 \\
3240\end{array}$ & $\begin{array}{l}2.29 \\
1.53 \\
.58 \\
0\end{array}$ \\
\hline \multicolumn{13}{|c|}{1971 WATER YEAR } \\
\hline $\begin{array}{l}4-26-71 \\
4-27-71\end{array}$ & $0^{.03}$ & $0^{.49}$ & 257 & $\begin{array}{r}1.33 \\
.07\end{array}$ & 272 & $0^{.65}$ & 903 & $0^{1.19}$ & 755 & * & 1160 & $T^{.23}$ \\
\hline $\begin{array}{l}5-21-71 \\
5-22-71\end{array}$ & $\begin{array}{l}0 \\
0\end{array}$ & $\begin{array}{l}.29 \\
.10\end{array}$ & 547 & $\begin{array}{l}.94 \\
.34\end{array}$ & 440 & $\begin{array}{l}.61 \\
.48\end{array}$ & 1060 & $\begin{array}{r}1.11 \\
.72\end{array}$ & 1120 & 1400 & 2760 & $\begin{array}{r}1.01 \\
.63\end{array}$ \\
\hline $\begin{array}{l}5-31-71 \\
6-01-71\end{array}$ & $0^{.10}$ & $0^{.37}$ & 437 & 1.23 & 845 & .24 & 2370 & 0 & 1740 & 892 & 954 & \\
\hline $6-10-71$ & 0 & * & & .64 & & .41 & & 1.00 & 29 & 800 & & 1.44 \\
\hline ו-8-04-7 & .05 & 1.58 & 4 & 1.33 & 8 & .36 & 8 & .40 & & & & .13 \\
\hline $9-04-71$ & .44 & 1.20 & $<1$ & * & * & 1.20 & 8 & .54 & & & & 45 \\
\hline
\end{tabular}

\begin{tabular}{|c|c|c|c|c|c|c|c|c|c|c|c|c|}
\hline $\begin{array}{l}10-29-71 \\
10-30-71 \\
10-31-71 \\
11-01-71 \\
11-02-71\end{array}$ & " & 88 & $\begin{array}{l}2.18 \\
.56 \\
0.07 \\
0 \\
0\end{array}$ & * & $\begin{array}{l}2.37 \\
.41 \\
0.13 \\
0\end{array}$ & $\begin{array}{r}16 \\
33 \\
603\end{array}$ & $\begin{array}{l}1.72 \\
.25 \\
1.42 \\
0\end{array}$ & $\begin{array}{r}18 \\
18 \\
1480\end{array}$ & $\begin{array}{l}1.71 \\
.36 \\
0.46 \\
1.46\end{array}$ & $\begin{array}{r}22 \\
27 \\
1350\end{array}$ & $\begin{array}{l}16 \\
24\end{array}$ & $\begin{array}{l}40 \\
28\end{array}$ \\
\hline $\begin{array}{l}5-06-72 \\
5-07-72\end{array}$ & : & & $\begin{array}{r}1.15 \\
.01\end{array}$ & 31 & $0^{1.26}$ & 112 & $0^{.78}$ & 768 & $\begin{array}{l}.03 \\
.15\end{array}$ & 854 & 1070 & 3240 \\
\hline $6-06-72$ & * & & .10 & & 0 & & 2.19 & 18 & 0 & & & \\
\hline $\begin{array}{l}6-13-72 \\
6-14-72\end{array}$ & $\begin{array}{l}.15 \\
.80\end{array}$ & $<1$ & $\begin{array}{l}.13 \\
1.12\end{array}$ & $<1$ & $\begin{array}{l}.20 \\
1.51\end{array}$ & 59 & $\begin{array}{r}.37 \\
1.42\end{array}$ & 85 & $\begin{array}{l}.02 \\
.41\end{array}$ & & & \\
\hline $7-11-72$ & 1.77 & 4.3 & 1.19 & 49 & .98 & 35 & .11 & & .18 & & & \\
\hline $\begin{array}{l}7-17-72 \\
7-18-72\end{array}$ & $\begin{array}{l}1.88 \\
0\end{array}$ & 22 & $\begin{array}{l}2.00 \\
0\end{array}$ & 105 & $\begin{array}{r}1.67 \\
.02\end{array}$ & 126 & $\begin{array}{r}1.47 \\
.03\end{array}$ & 81 & $0^{.75}$ & 64 & 177 & 303 \\
\hline $\begin{array}{l}7-24-72 \\
7-25-72\end{array}$ & $0^{.49}$ & & $0^{.32}$ & & $\begin{array}{l}1.45 \\
0\end{array}$ & 16 & $0^{1.38}$ & 423 & $0^{.23}$ & 259 & 139 & 225 \\
\hline $\begin{array}{l}8-02-72 \\
8-03-72 \\
8-04-72\end{array}$ & $\begin{array}{l}1.24 \\
0.60\end{array}$ & 315 & $\begin{array}{l}.71 \\
0.50 \\
0\end{array}$ & 831 & $\begin{array}{l}1.30 \\
.73\end{array}$ & 1020 & $\begin{array}{l}.78 \\
1.68 \\
0\end{array}$ & 2640 & $0^{.59}$ & 2720 & 1760 & 1860 \\
\hline $\begin{array}{l}8-24-72 \\
8-25-72\end{array}$ & $\begin{array}{r}2.30 \\
.10\end{array}$ & 10 & $\begin{array}{r}1.61 \\
.11\end{array}$ & 61 & $\begin{array}{r}1.28 \\
.07\end{array}$ & 64 & $\begin{array}{r}1.35 \\
.01\end{array}$ & 20 & $0^{.52}$ & & & \\
\hline $\begin{array}{l}9-06-72 \\
9-07-72 \\
9-08-72\end{array}$ & $\begin{array}{l}0 \\
1.19 \\
0\end{array}$ & 8 & $\begin{array}{l}0 \\
1.23 \\
0\end{array}$ & 66 & $l_{0}^{.04}$ & 85 & $0^{.28}$ & 2690 & $\begin{array}{l}2.50 \\
0^{2.32}\end{array}$ & 3470 & 3330 & 5260 \\
\hline $\begin{array}{l}9-13-72 \\
9-14-72 \\
9-15-72\end{array}$ & $0_{0}^{.93}$ & 26 & $\begin{array}{l}1.01 \\
0 \\
0\end{array}$ & 223 & $\begin{array}{l}1.60 \\
0 \\
0\end{array}$ & $\begin{array}{l}285 \\
249\end{array}$ & $0_{0}^{.35}$ & 2010 & $0_{0}^{.11}$ & 1440 & 908 & 889 \\
\hline
\end{tabular}

1973 WATER YEAR

$\begin{array}{llllllll}10-22-72 & 1.27 & 2 & 1.30 & 13 & 1.32 & 32 & 0 \\ 10-23-72 & 0 & & 0 & & 0 & 1.05\end{array}$

$11-09-72 \quad 0^{.87}$

$\begin{array}{llll}11-12-72 & .42 & 1.00 \\ 11-13-72 & 1.00 & 136 & 1.06 \\ 11-14-72 & \$\end{array}$

3-30-73

$3-31-73 \quad .59$

$\begin{array}{ll}3-31-73 & 1.22 \\ 4-01-73 & 0\end{array}$

$4-14-73 \quad 55$

$\begin{array}{ll}4-15-73 & 1.14 \\ 4-16-73 & 0\end{array}$

$\begin{array}{ll}4-30-73 & .71 \\ 5-01-73 & .07\end{array}$
$0^{.81}$

$0^{.89}$

358

.16

1.44

769

$i_{0}^{.63} \quad 1150$

$0^{.64}$

$\begin{array}{lll}1.67 & 1120 & .10 \\ 0 & & .98\end{array}$

1.37
1.11
.07

137
3360

$440^{.38}$

$1210 \quad 0^{.42}$

$2350 \quad .17$
.66

1180

2990

$0^{.43} \quad 3860$

$593 \quad \begin{aligned} & .14 \\ & 1.07\end{aligned}$

$3110 \quad 0^{1.07}$

1.71
3540

2760

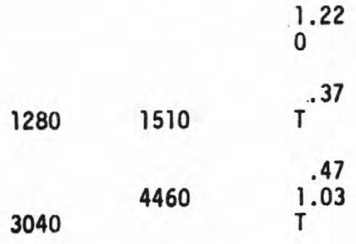

5110

4160

5110

$T^{.67}$

.41
1.76

1.16

1.22
.01 
Table 3.--Soldler Creek Ralnfall and Peak-Flow Data (Concluded).

1973 WATER YEAR--Continued

Goff Bancroft Soldier Circleville $\underline{\text { St. Clere }} \underline{\text { Delia }} \underline{\text { Topeka }} \underline{\text { Topeka NWs }}$ I/

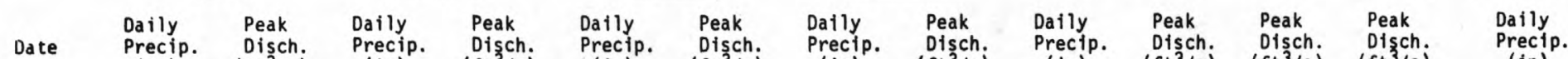

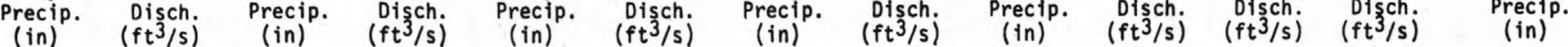

\begin{tabular}{|c|c|c|c|c|c|c|c|c|c|c|c|c|c|}
\hline $\begin{array}{l}5-06-73 \\
5-07-73\end{array}$ & $\begin{array}{r}1.52 \\
.37\end{array}$ & 347 & $\begin{array}{r}1.50 \\
.42\end{array}$ & $\begin{array}{l}317 \\
980\end{array}$ & $\begin{array}{r}1.63 \\
.34\end{array}$ & $\begin{array}{l}555 \\
983\end{array}$ & $\begin{array}{r}1.63 \\
.16\end{array}$ & $\begin{array}{l}2120 \\
2020\end{array}$ & $\begin{array}{r}1.26 \\
.30\end{array}$ & $\begin{array}{l}2430 \\
2370\end{array}$ & 2540 & 3890 & $\begin{array}{r}1.18 \\
.01\end{array}$ \\
\hline $\begin{array}{l}5-26-73 \\
5-27-73\end{array}$ & $\begin{array}{l}.96 \\
.05\end{array}$ & .6 & $\begin{array}{r}1.31 \\
.05\end{array}$ & 36 & $\begin{array}{l}.78 \\
.01\end{array}$ & 26 & $0^{.86}$ & 35 & $0^{.30}$ & & & & ${ }_{T}^{1.60}$ \\
\hline $\begin{array}{l}6-03-73 \\
6-04-73 \\
6-05-73\end{array}$ & $\begin{array}{l}1.02 \\
. .22\end{array}$ & 7 & $0^{.98}$ & 43 & $\begin{array}{l}1.35 \\
0^{.43}\end{array}$ & $\begin{array}{l}97 \\
42\end{array}$ & $\begin{array}{l}1.10 \\
. .30\end{array}$ & $\begin{array}{l}130 \\
216\end{array}$ & $0^{.06}$ & $\begin{array}{l}169 \\
230\end{array}$ & 2720 & 3730 & $\begin{array}{r}2.21 \\
.39 \\
.05\end{array}$ \\
\hline $\begin{array}{l}7-02-73 \\
7-03-73\end{array}$ & $\begin{array}{l}.21 \\
.03\end{array}$ & & $\begin{array}{l}.97 \\
.64\end{array}$ & $\begin{array}{l}3 \\
4\end{array}$ & $\begin{array}{r}1.15 \\
.71\end{array}$ & 19 & $\begin{array}{r}1.29 \\
.16\end{array}$ & 26 & $0^{.76}$ & 62 & 61 & & $0^{1.63}$ \\
\hline $\begin{array}{l}7-18-73 \\
7-19-73 \\
7-20-73\end{array}$ & $\begin{array}{l}.03 \\
.06 \\
.13\end{array}$ & 50 & $\begin{array}{l}.30 \\
1.25 \\
1.52\end{array}$ & 252 & $\begin{array}{r}.37 \\
1.83 \\
.81\end{array}$ & 240 & $\begin{array}{r}.96 \\
1.47 \\
1.21\end{array}$ & $\begin{array}{r}88 \\
762\end{array}$ & $\begin{array}{r}.56 \\
1.64 \\
.88\end{array}$ & 853 & 3510 & 10100 & $\begin{array}{l}2.62 \\
2.11 \\
2.44\end{array}$ \\
\hline $7-25-73$ & 0 & & 0 & & 0 & & .01 & & .01 & 161 & 1470 & 3400 & $T$ \\
\hline $\begin{array}{l}8-07-73 \\
8-08-73 \\
8-09-73\end{array}$ & $\begin{array}{r}3.90 \\
.65 \\
.07\end{array}$ & 930 & $\begin{array}{r}3.38 \\
.70 \\
.54\end{array}$ & 2380 & $\begin{array}{r}3.19 \\
.99 \\
.34\end{array}$ & 1980 & $\begin{array}{r}1.61 \\
1.23 \\
.16\end{array}$ & 4410 & $\begin{array}{l}.04 \\
.64 \\
.11\end{array}$ & 3900 & 2590 & 2440 & $\begin{array}{l}.23 \\
.42 \\
.10\end{array}$ \\
\hline $\begin{array}{l}9-07-73 \\
9-08-73 \\
9-09-73\end{array}$ & $0^{.83}$ & 46 & $0^{.85}$ & 38 & $0^{.79}$ & 100 & $\begin{array}{l}.96 \\
.36 \\
.02\end{array}$ & 44 & $\begin{array}{r}1.12 \\
.63 \\
.10\end{array}$ & * & 109 & 136 & $\begin{array}{l}.61 \\
.11 \\
.04\end{array}$ \\
\hline $\begin{array}{l}9-16-73 \\
9-17-73\end{array}$ & $\begin{array}{r}1.11 \\
.04\end{array}$ & 6 & $\begin{array}{l}.86 \\
.04\end{array}$ & 105 & $\begin{array}{l}.70 \\
.04\end{array}$ & 171 & $\begin{array}{l}.51 \\
.03\end{array}$ & 164 & $\begin{array}{l}.52 \\
.02\end{array}$ & 163 & 168 & * & $\begin{array}{l}.58 \\
.01\end{array}$ \\
\hline $\begin{array}{l}9-23-73 \\
9-24-73 \\
9-25-73 \\
9-26-73 \\
9-27-73 \\
9-28-73\end{array}$ & $\begin{array}{l}1.30 \\
1.21 \\
.03 \\
1.86 \\
.39 \\
.07\end{array}$ & $\begin{array}{r}97 \\
118 \\
-250 \\
237\end{array}$ & $\begin{array}{l}1.35 \\
1.04 \\
0 \\
2.28 \\
0.46\end{array}$ & $\begin{array}{l}509 \\
452 \\
\\
881 \\
624\end{array}$ & $\begin{array}{l}1.04 \\
1.06 \\
.02 \\
2.36 \\
. .49 \\
0 .\end{array}$ & $\begin{array}{r}572 \\
552 \\
1130 \\
773\end{array}$ & $\begin{array}{l}1.39 \\
1.15 \\
0 \\
2.52 \\
.29 \\
.05\end{array}$ & $\begin{array}{r}953 \\
1240 \\
4200 \\
2270\end{array}$ & $\begin{array}{r}1.04 \\
1.45 \\
.02 \\
2.68 \\
1.22 \\
.24\end{array}$ & $\begin{array}{r}930 \\
2060 \\
4700 \\
2980\end{array}$ & $\begin{array}{l}2630 \\
5460\end{array}$ & $11000+$ & $\begin{array}{r}3.74 \\
2.53 \\
.08 \\
2.21 \\
1.30 \\
.44\end{array}$ \\
\hline
\end{tabular}

\begin{tabular}{|c|c|c|c|c|c|c|c|c|c|c|c|c|c|}
\hline $\begin{array}{l}10-10-73 \\
10-11-73\end{array}$ & $\begin{array}{l}3.61 \\
1.14\end{array}$ & $\begin{array}{l}764 \\
394\end{array}$ & $\begin{array}{l}3.27 \\
1.36\end{array}$ & $\begin{array}{l}1400 \\
1290\end{array}$ & $\begin{array}{l}2.60 \\
1.40\end{array}$ & $\begin{array}{l}1450 \\
1510\end{array}$ & $\begin{array}{l}3.16 \\
1.05\end{array}$ & 5310 & $\begin{array}{r}2.77 \\
.82\end{array}$ & 6540 & 7020 & $20800+$ & $\begin{array}{r}3.16 \\
.88\end{array}$ \\
\hline $12-04-73$ & & & & & & & & 1390 & & 1890 & 2700 & 4860 & 1.72 \\
\hline $\begin{array}{l}4-20-74 \\
4-21-74\end{array}$ & " & 452 & $\begin{array}{r}2.79 \\
.66\end{array}$ & $\begin{array}{r}1200 \\
793\end{array}$ & $\begin{array}{r}2.43 \\
.02\end{array}$ & 1120 & $\begin{array}{l}2.99 \\
0\end{array}$ & 3520 & $\begin{array}{r}2.29 \\
.01\end{array}$ & 3720 & 3490 & 3500 & $0^{.63}$ \\
\hline $\begin{array}{l}4-29-74 \\
4-30-74\end{array}$ & * & 92 & $\begin{array}{l}1.23 \\
0\end{array}$ & $\begin{array}{l}177 \\
165\end{array}$ & $\begin{array}{l}1.29 \\
0\end{array}$ & $\begin{array}{l}244 \\
175\end{array}$ & $\begin{array}{l}2.33 \\
0\end{array}$ & 901 & $\begin{array}{r}1.30 \\
.01\end{array}$ & 1570 & 2240 & 3030 & ${ }_{T}^{1.56}$ \\
\hline $\begin{array}{l}6-05-74 \\
6-06-74 \\
6-07-74 \\
6-08-74 \\
6-09-74\end{array}$ & $\begin{array}{l}* \\
* \\
* \\
*\end{array}$ & $\begin{array}{r}11 \\
322\end{array}$ & $\begin{array}{r}.42 \\
.89 \\
.10 \\
2.17 \\
0\end{array}$ & $\begin{array}{r}43 \\
884\end{array}$ & $\begin{array}{l}.38 \\
.63 \\
.07 \\
2.31 \\
0\end{array}$ & $\begin{array}{r}22 \\
25 \\
999\end{array}$ & $\begin{array}{l}.14 \\
. .54 \\
0.60 \\
0\end{array}$ & $\begin{array}{r}42 \\
3740\end{array}$ & $\begin{array}{l}.34 \\
.86 \\
0 \\
1.92 \\
.01\end{array}$ & 3150 & $\begin{array}{l}1190 \\
3770\end{array}$ & $\begin{array}{l}1560 \\
3980\end{array}$ & $\begin{array}{l}.37 \\
.90 \\
T^{.90} \\
1.65 \\
T\end{array}$ \\
\hline $7-03-74$ & * & 0.2 & .78 & & 1.45 & 10 & .41 & & .39 & & & & 1.15 \\
\hline
\end{tabular}

+ Backwater from Kansas River. 

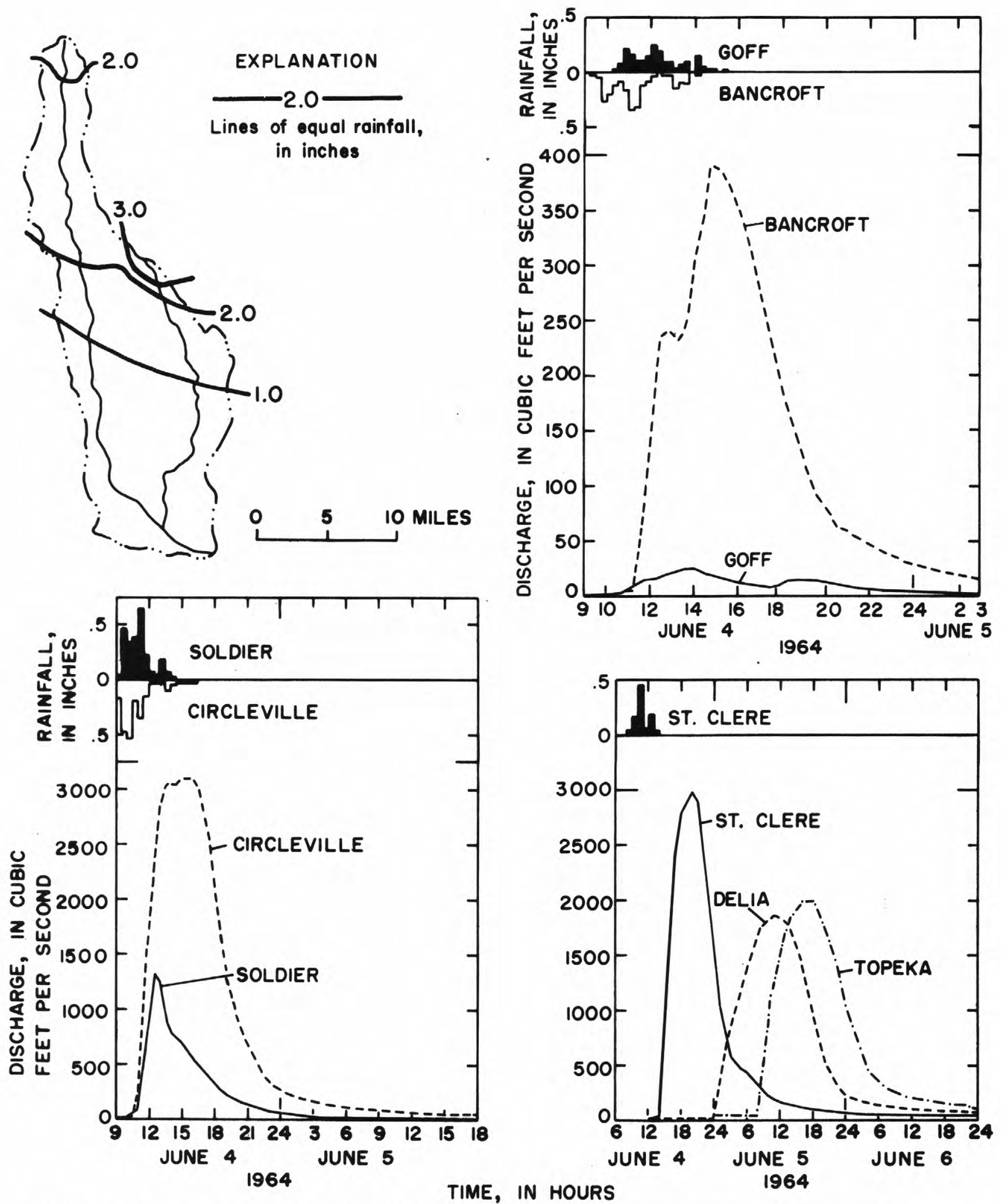

Figure 8.--Distribution of rainfall June 4, 1964, and resulting stream discharge. 

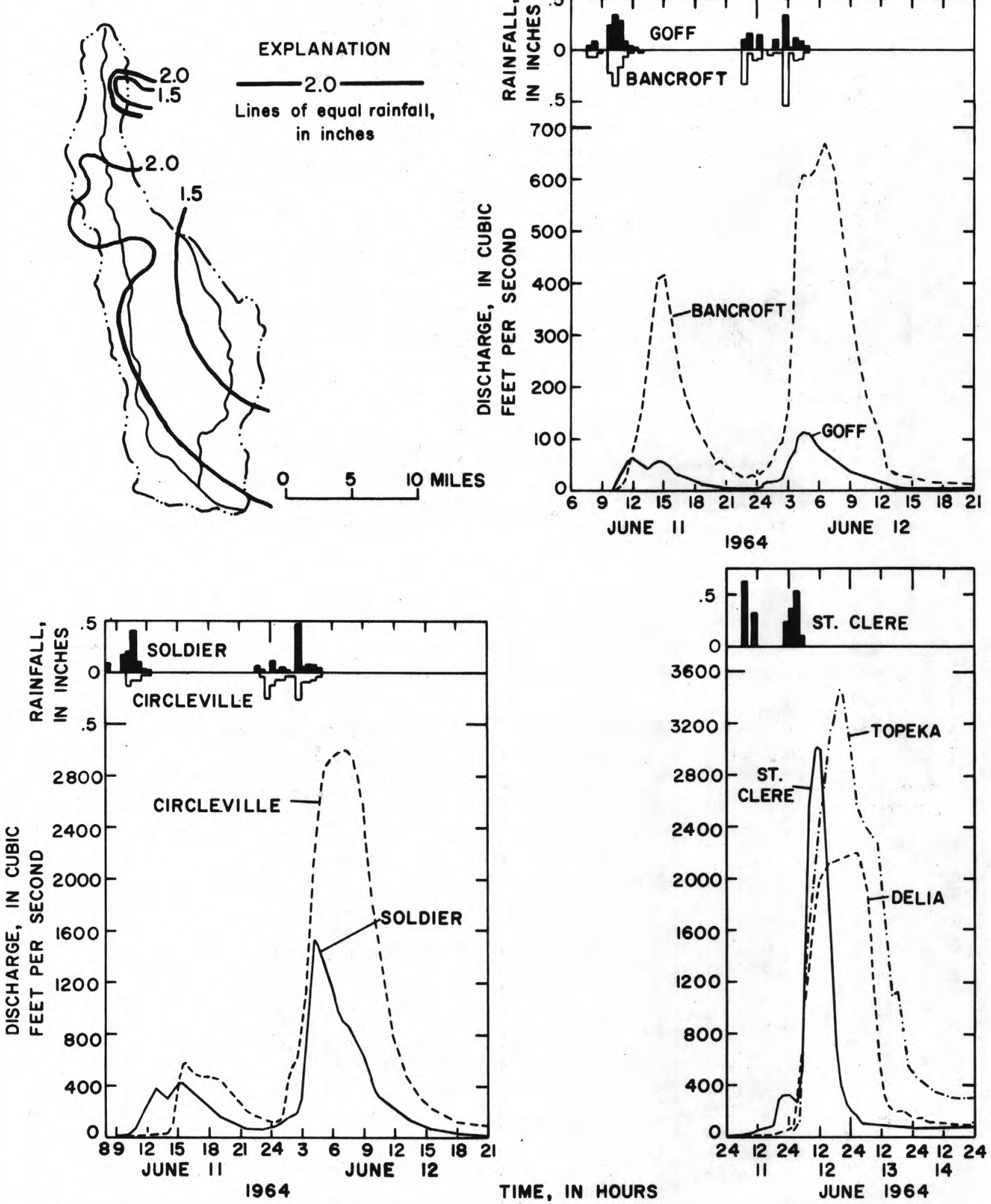

Figure 9.--Distribution of rainfall June 11-12, 1964, and resulting stream discharge. 

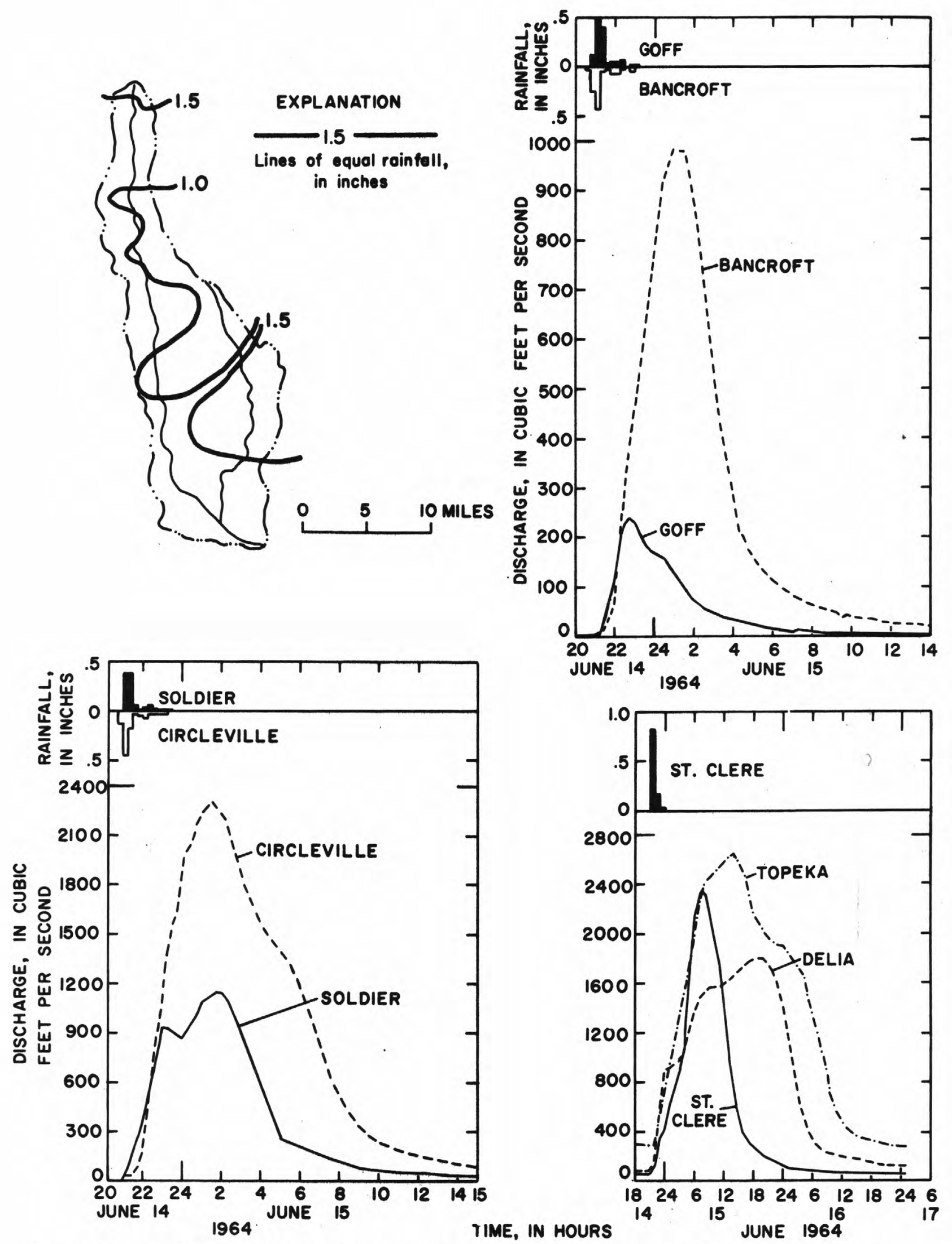

Figure 10.--Distribution of rainfall June 14-15, 1964, and resulting stream discharge. 


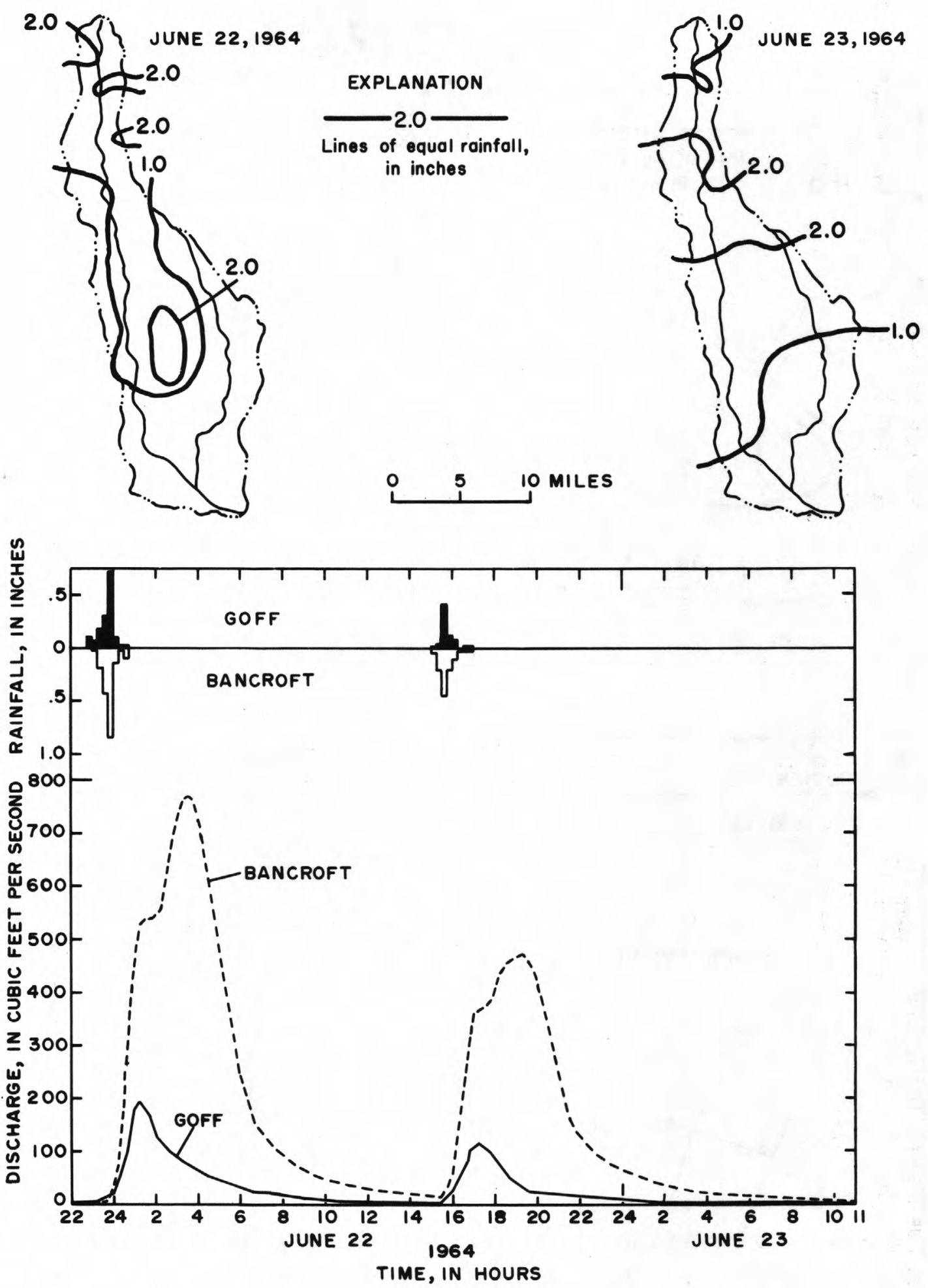

Figure 11.--Distribution of rainfall June 22-23, 1964, and resulting stream discharge. 

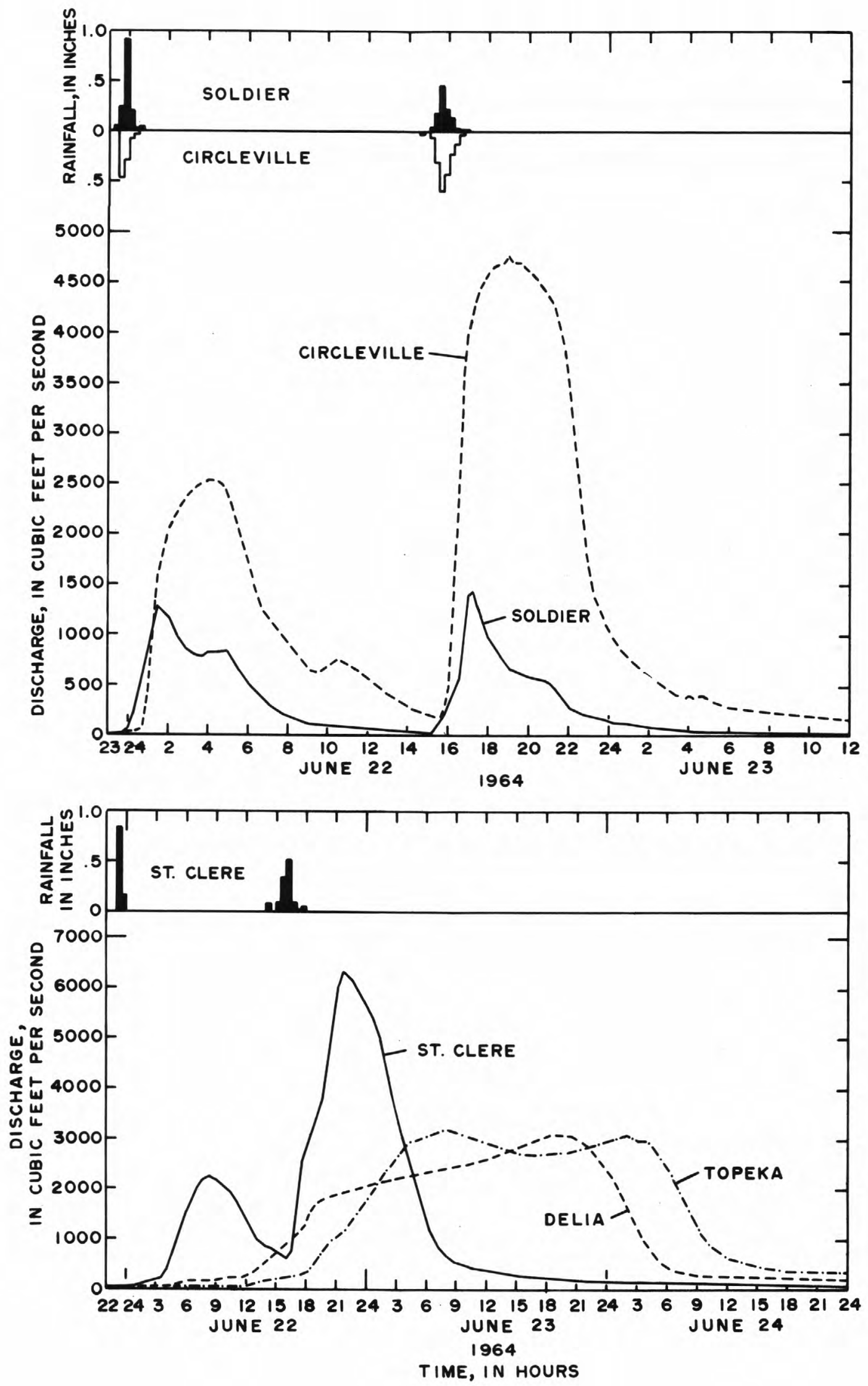

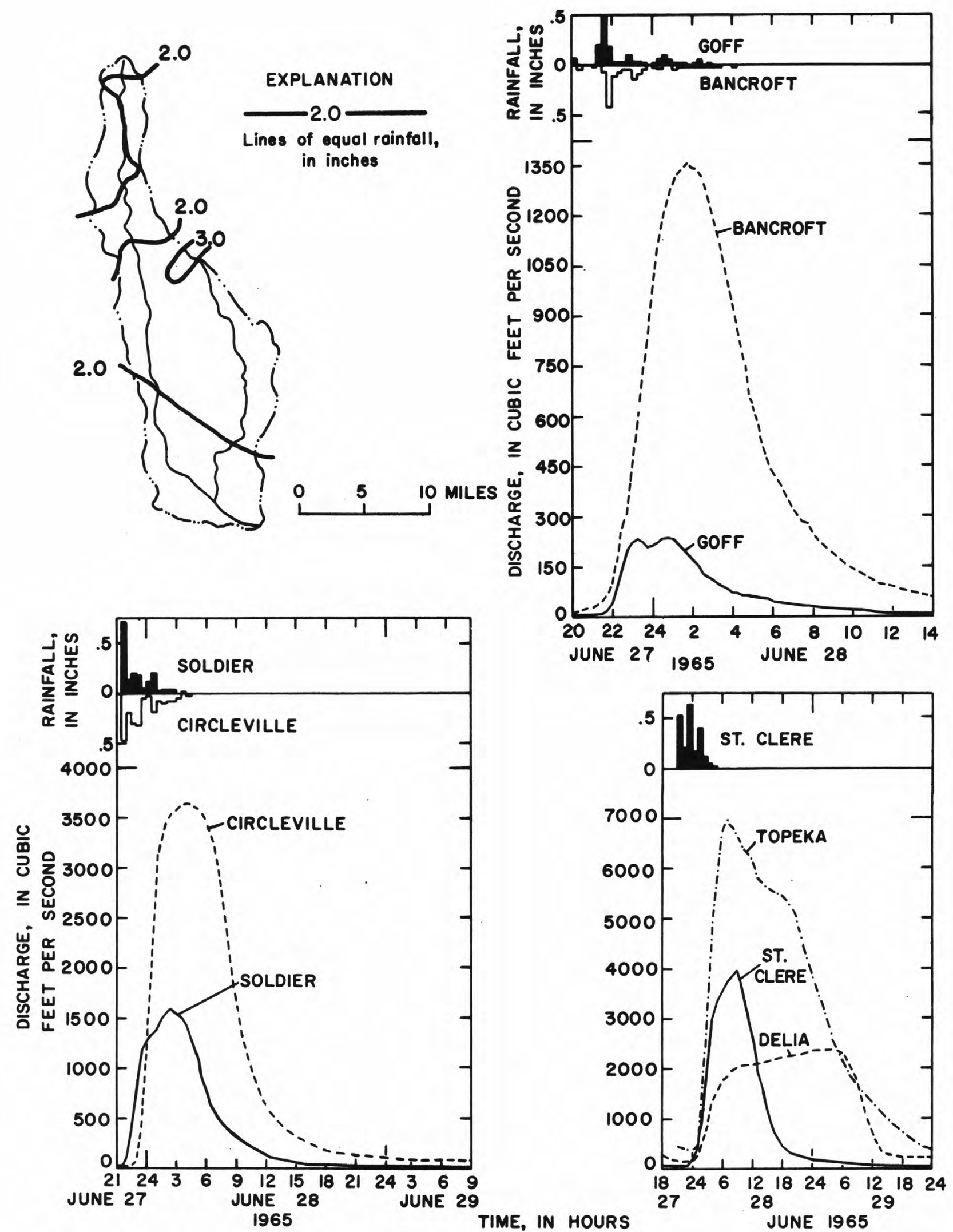

Figure 12.--Distribution of rainfall June 27-28, 1965, and resulting stream discharge. 

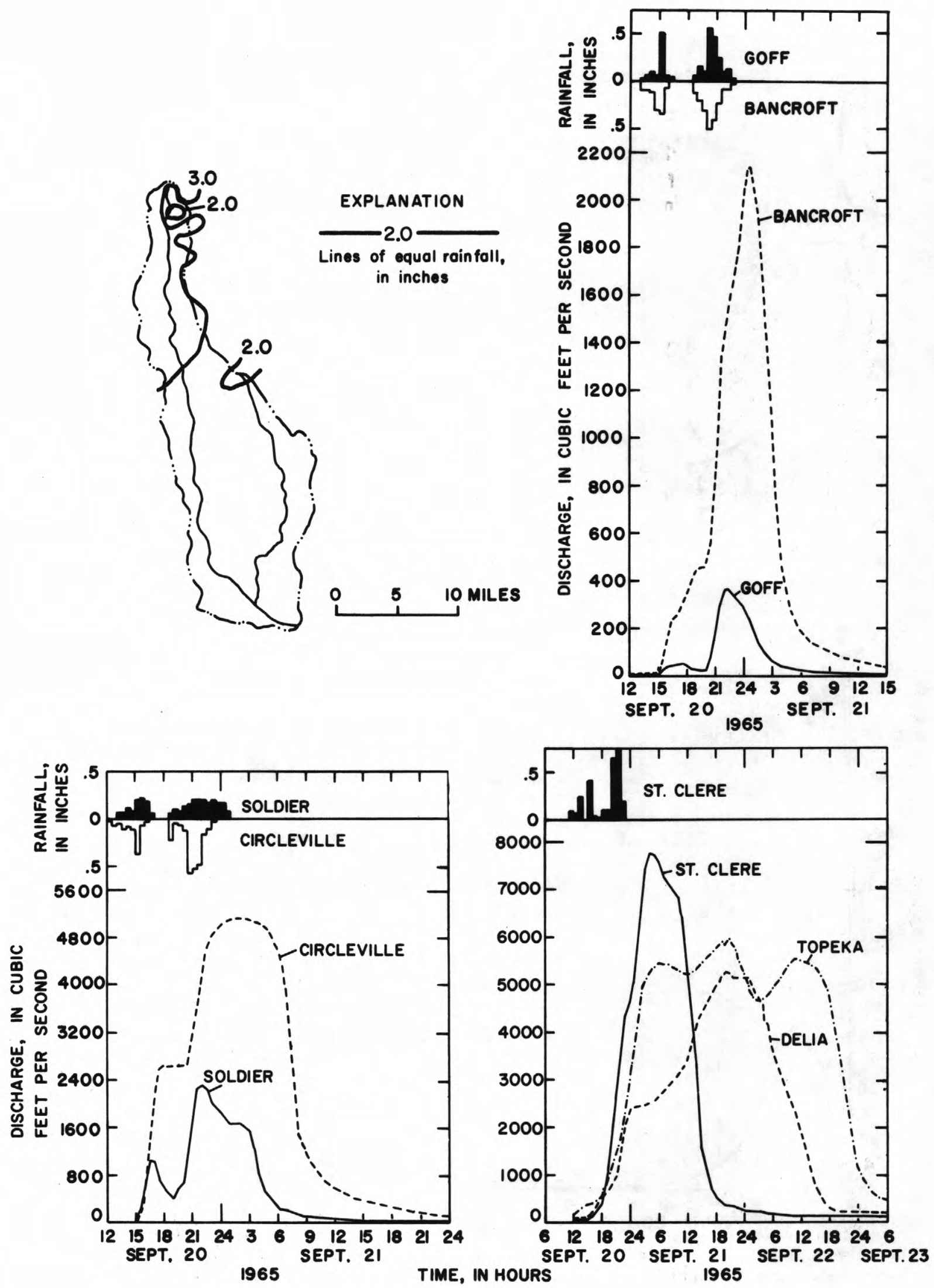

Figure 13.--Distribution of rainfall September 20-21, 1965, and resulting stream discharge. 

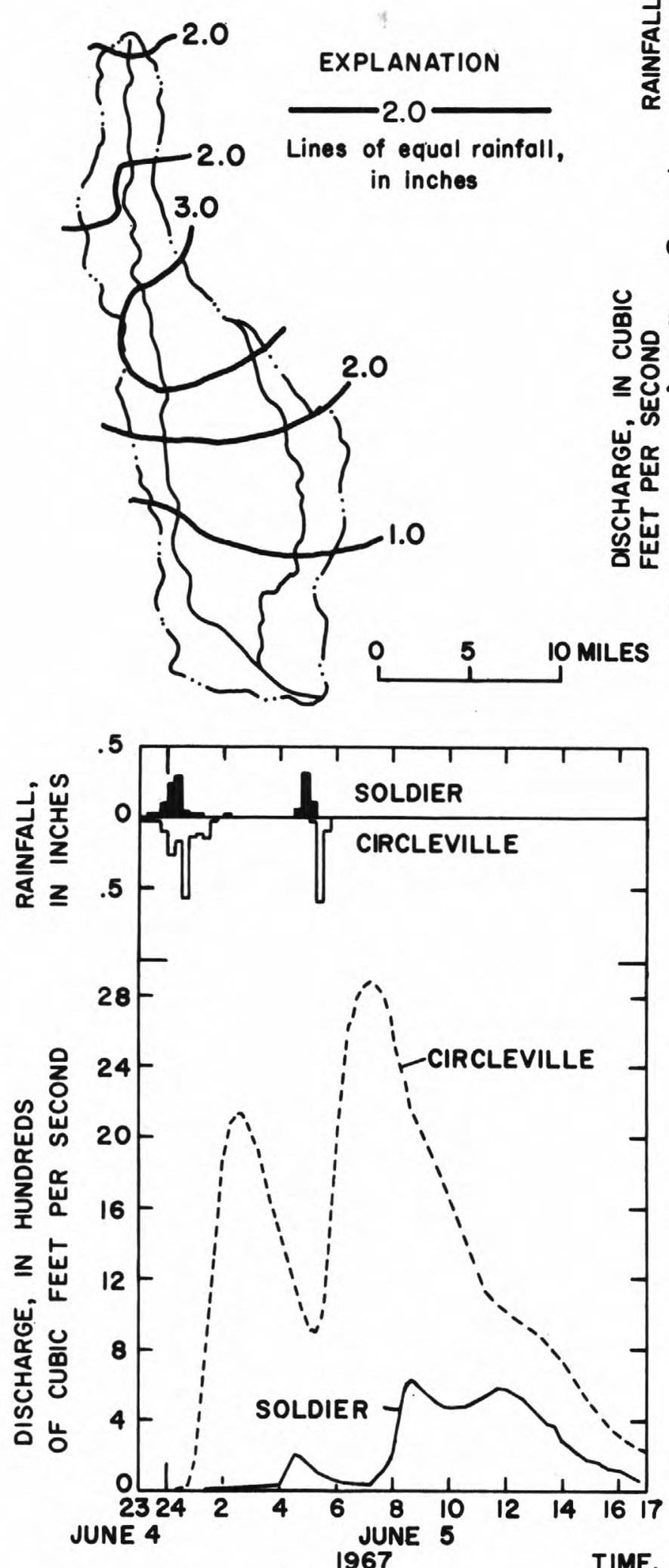

Figure 14.--Distribution of rainfall June 4-5, 1967, and resulting stream di scharge.

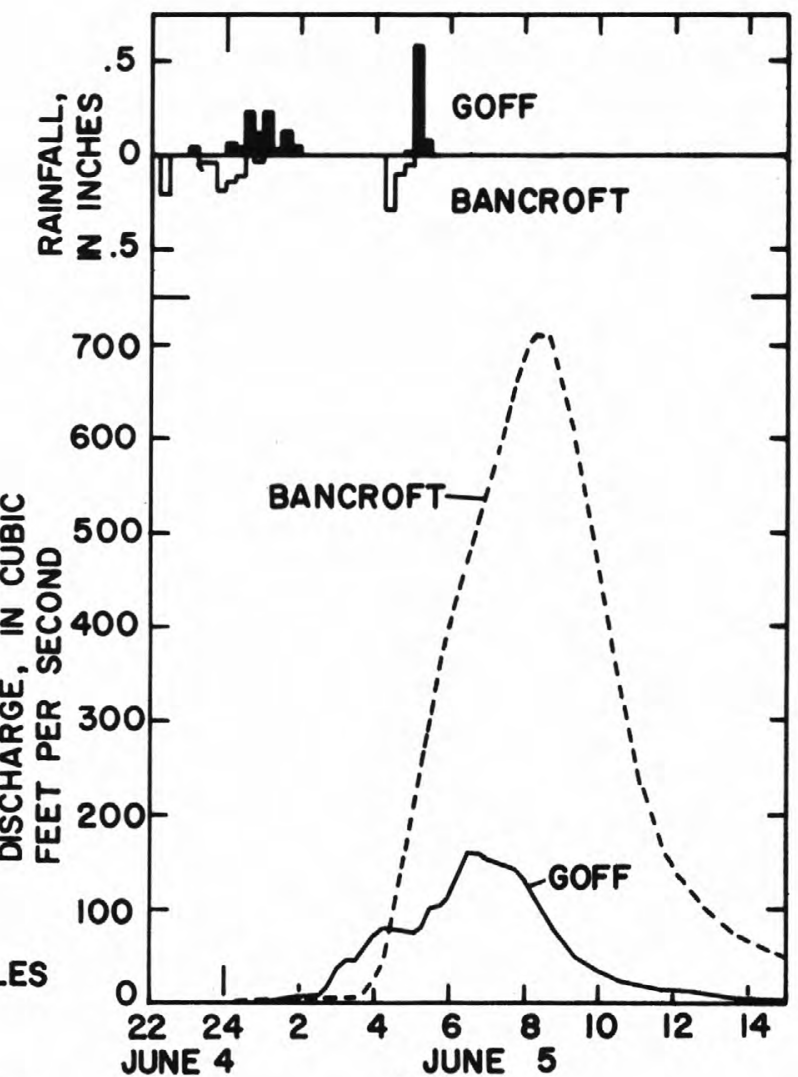

1967

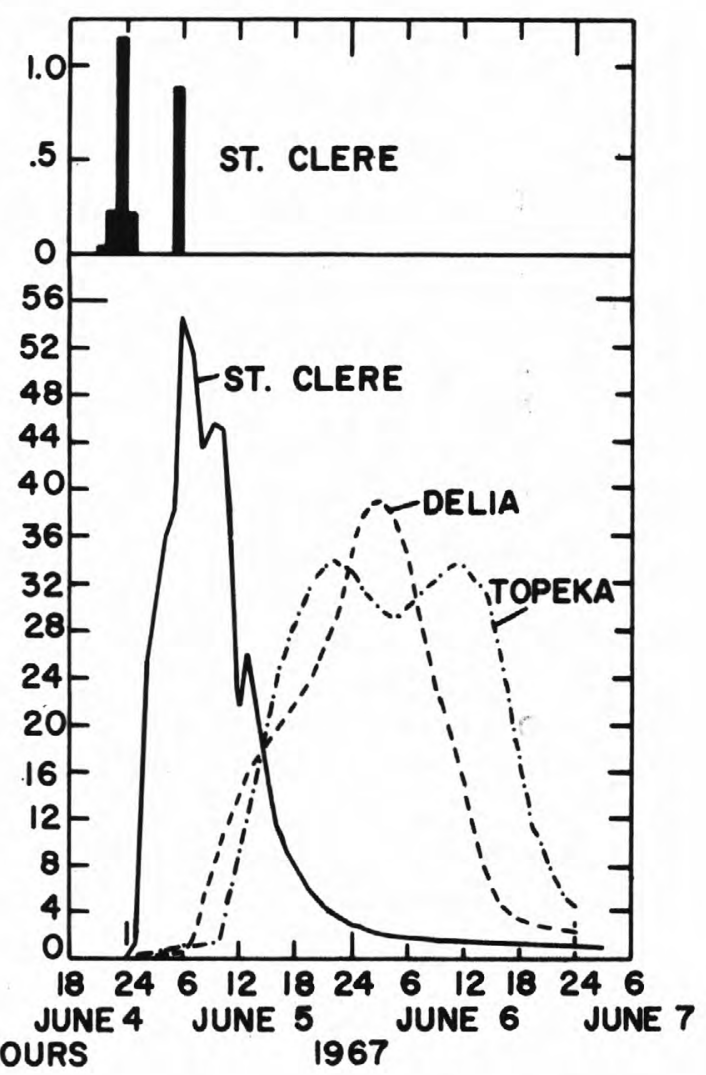




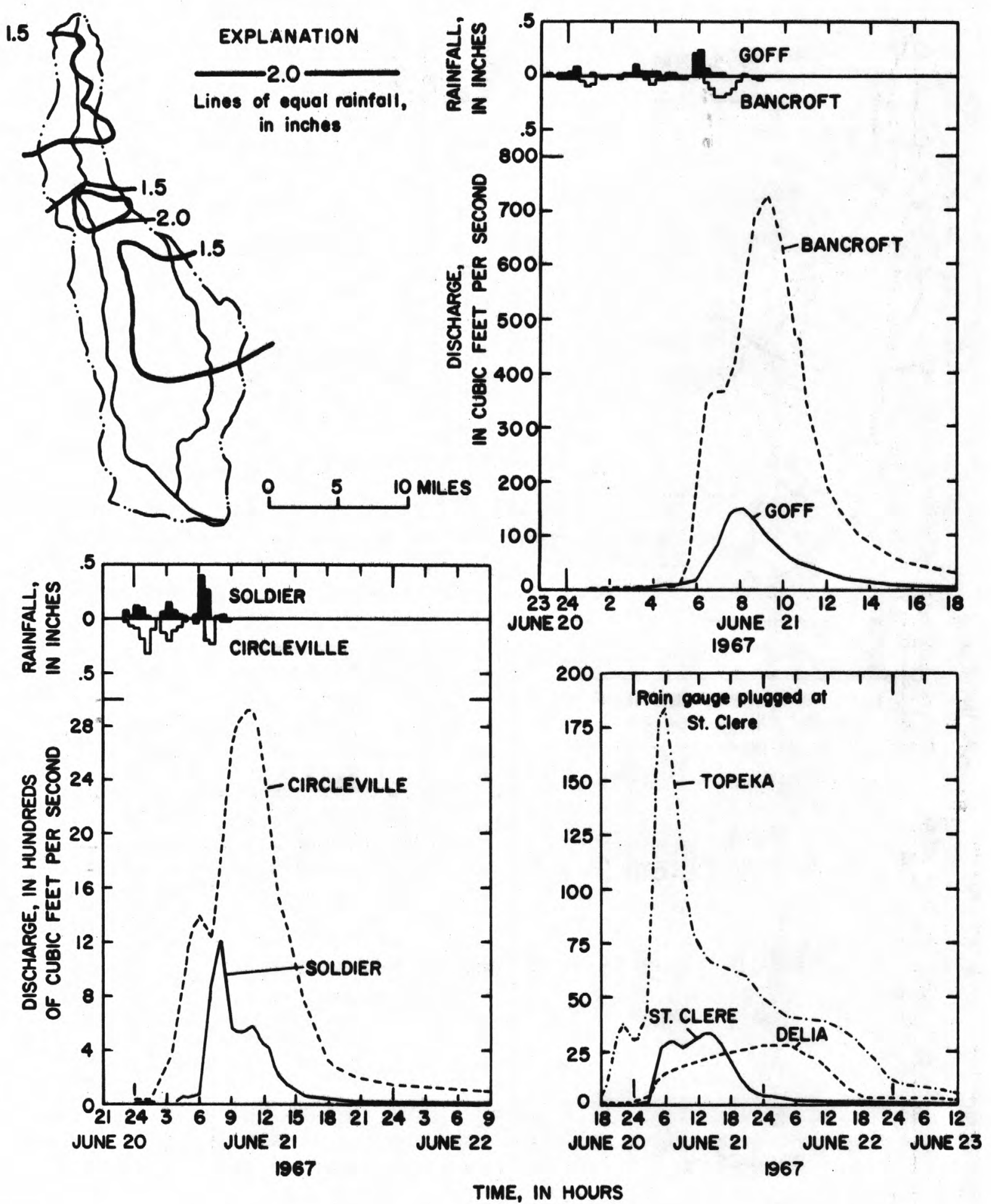

Figure 15.--Distribution of rainfall June 20-21, 1967, and resulting stream discharge. 

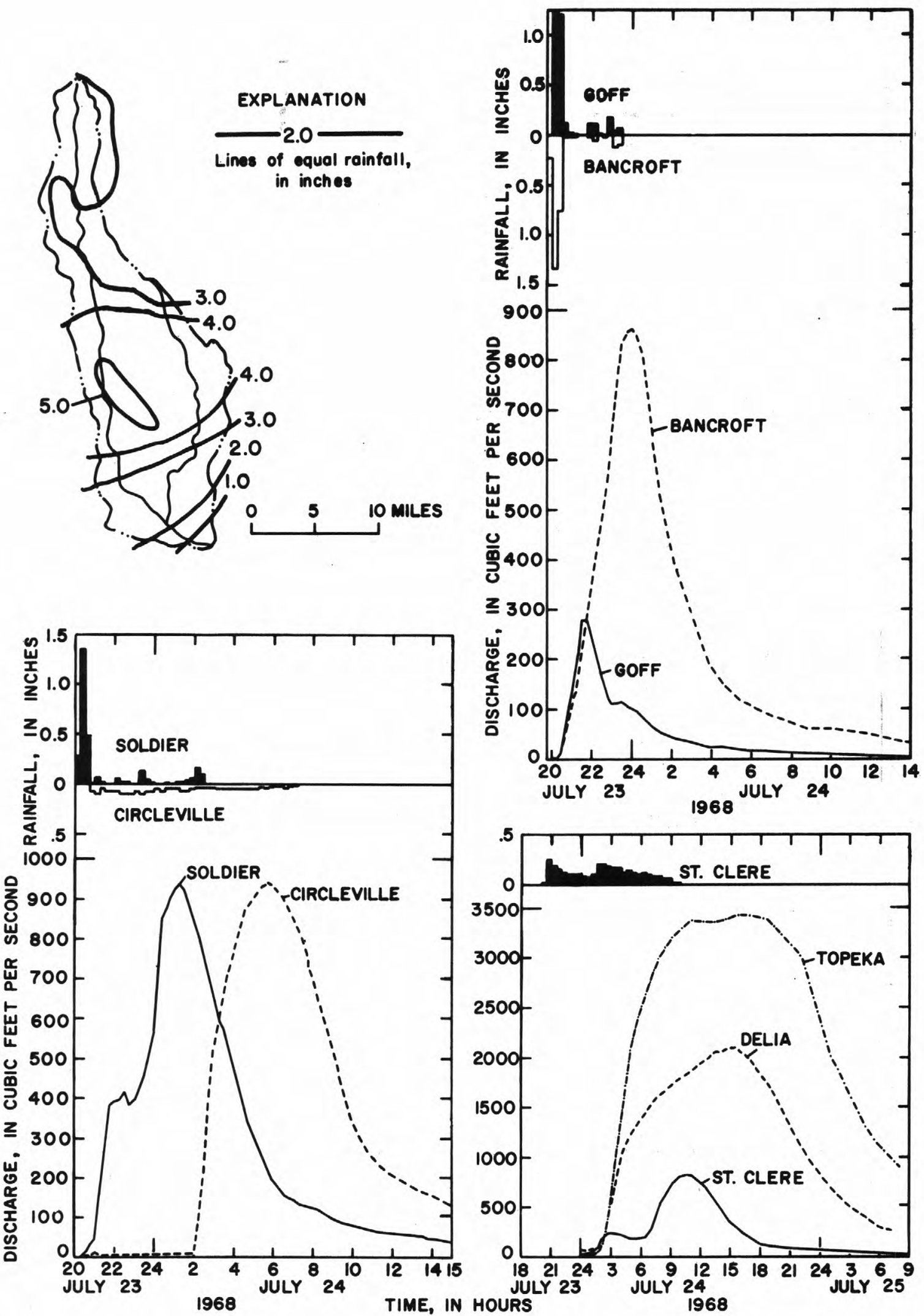

Figure 16.--Distribution of rainfall July 23-24, 1968, and resulting stream discharge. 

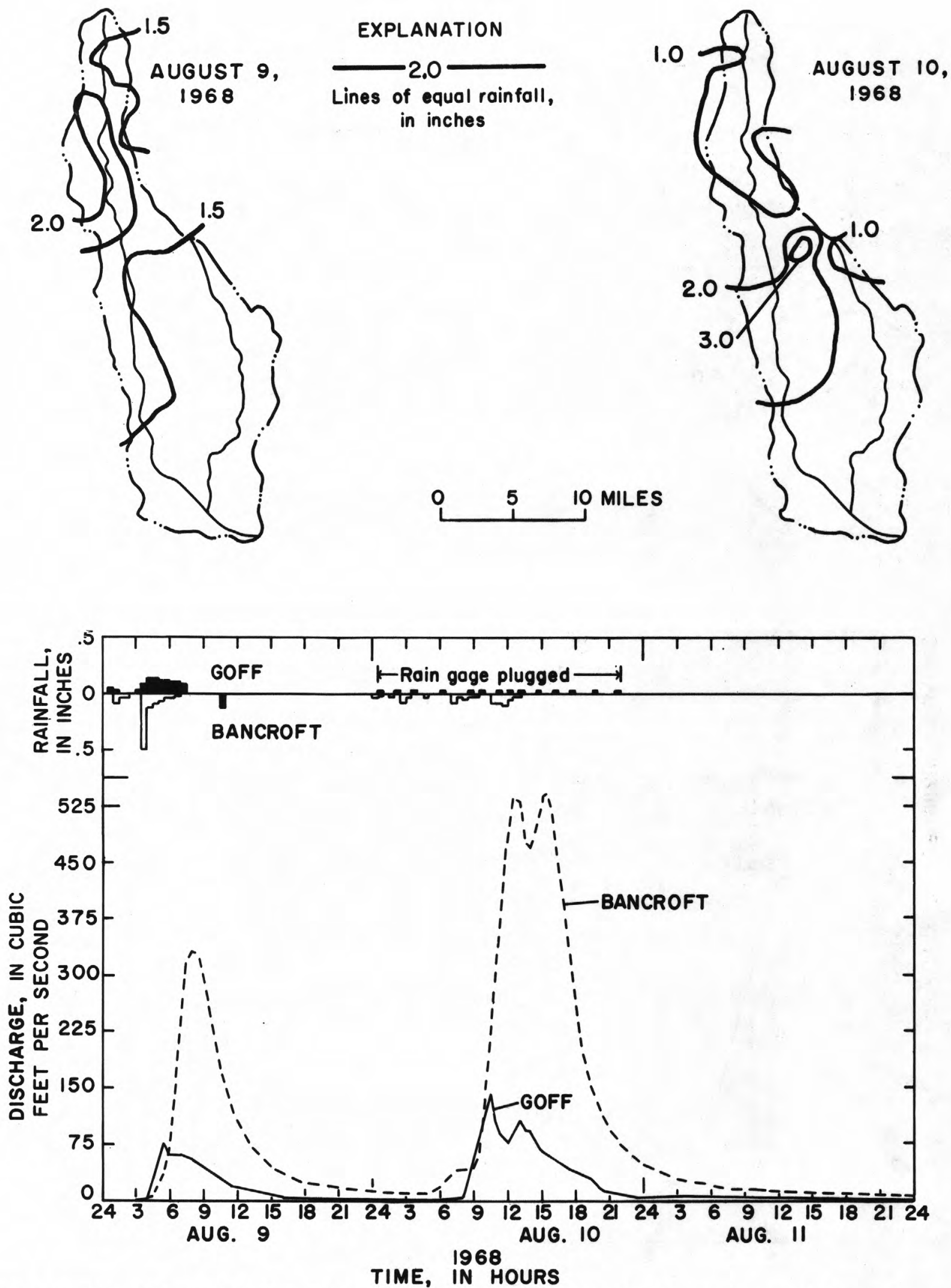

Figure 17.--Distribution of rainfall August 9-10, 1968, and resulting stream discharge. 


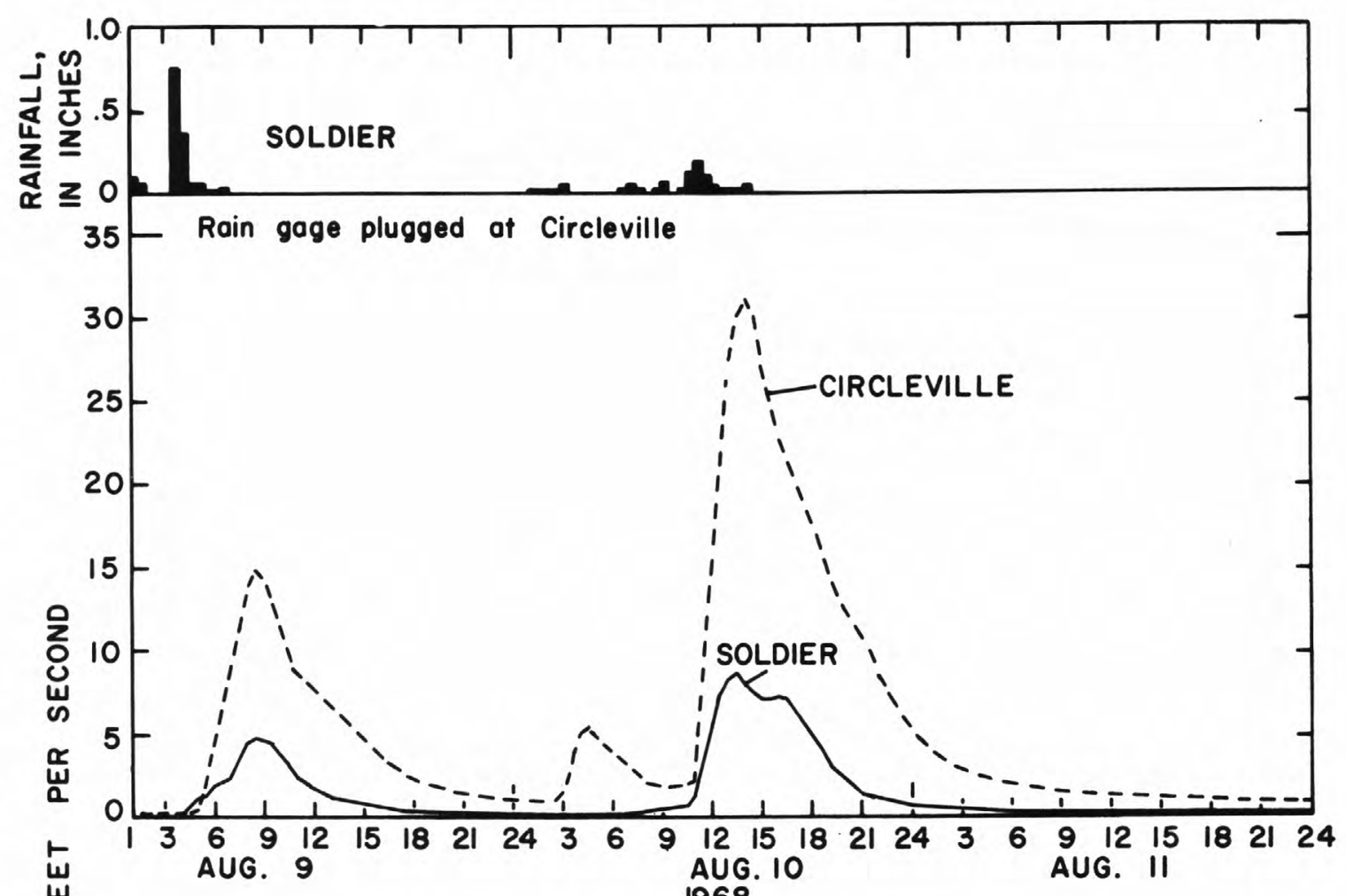

1968

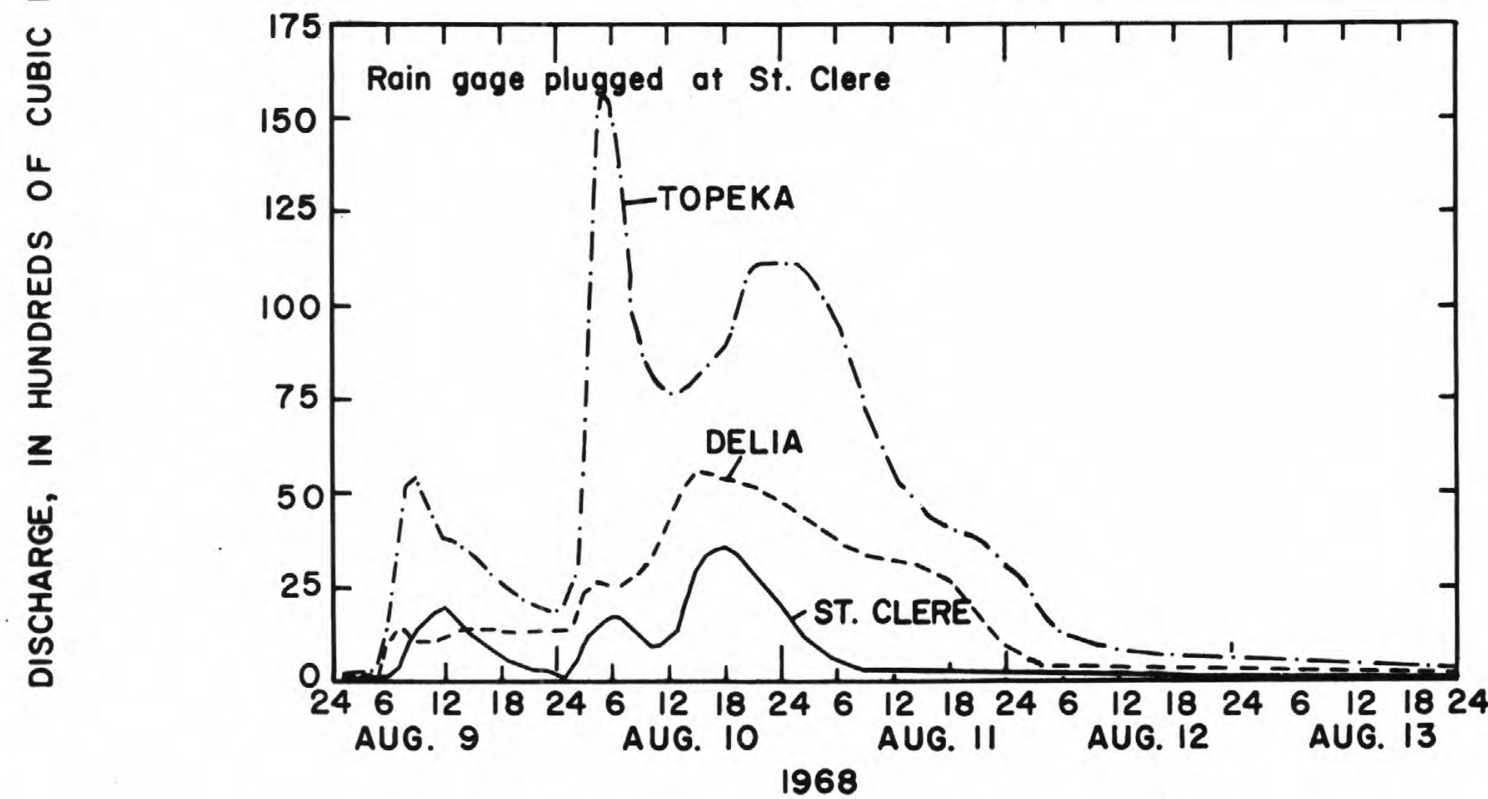

TIME, IN HOURS 

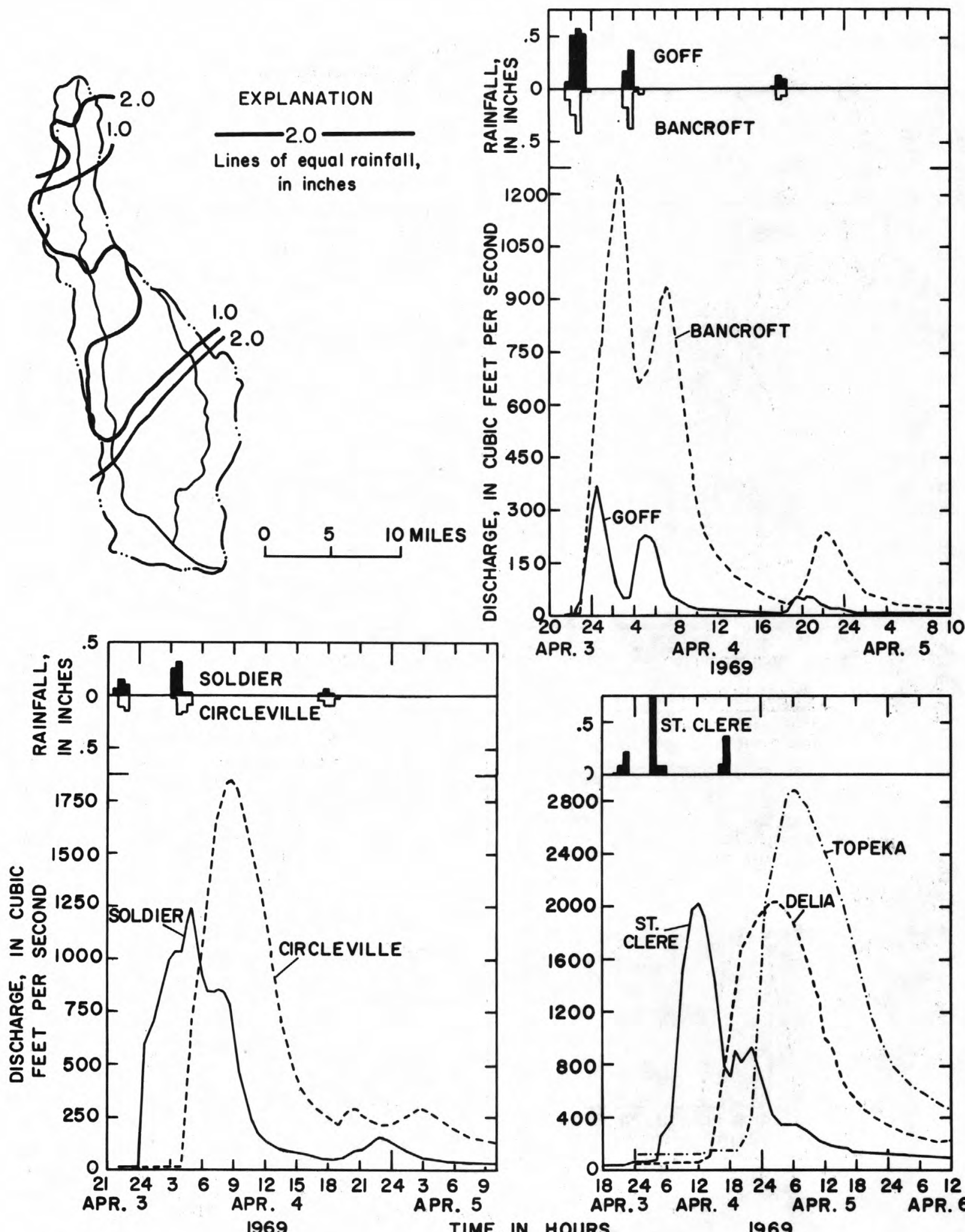

APR. 3

1969

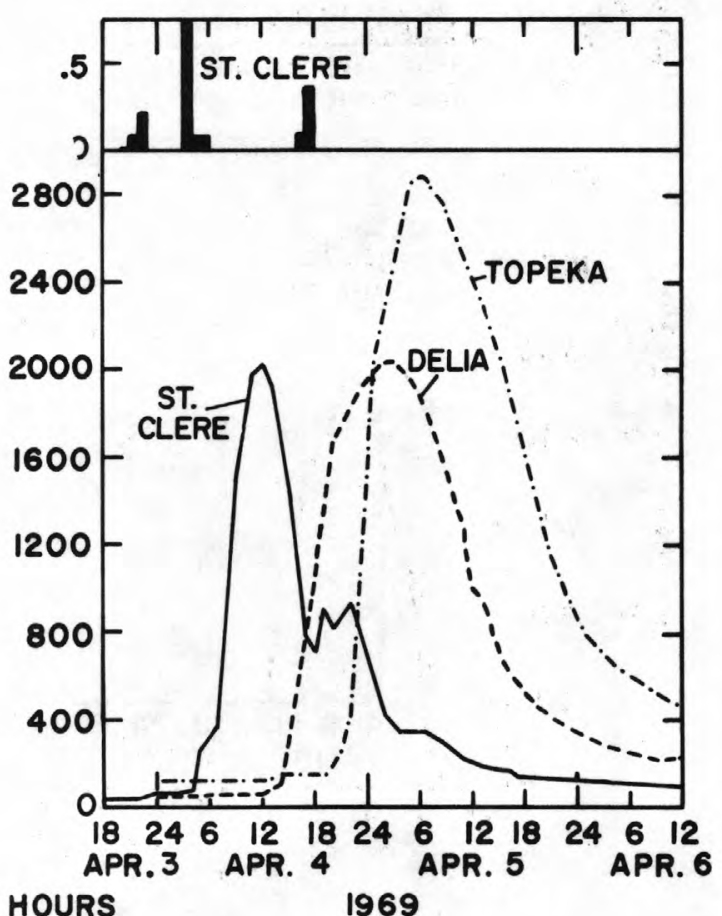

Figure 18.--Distribution of rainfall April 3-4, 1969, and resulting stream discharge. 

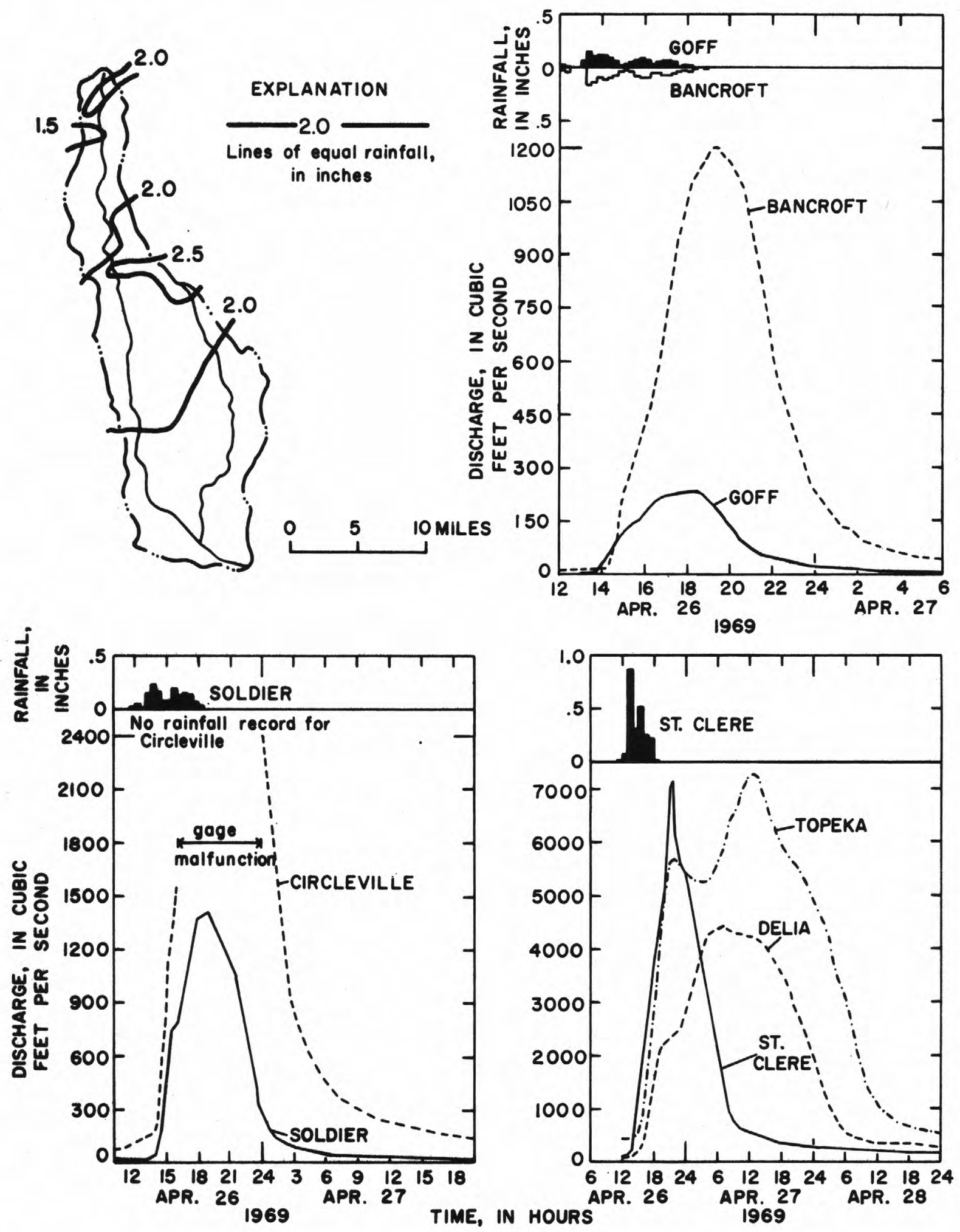

Figure 19.--Distribution of rainfall April 26, 1969, and resulting stream discharge. 

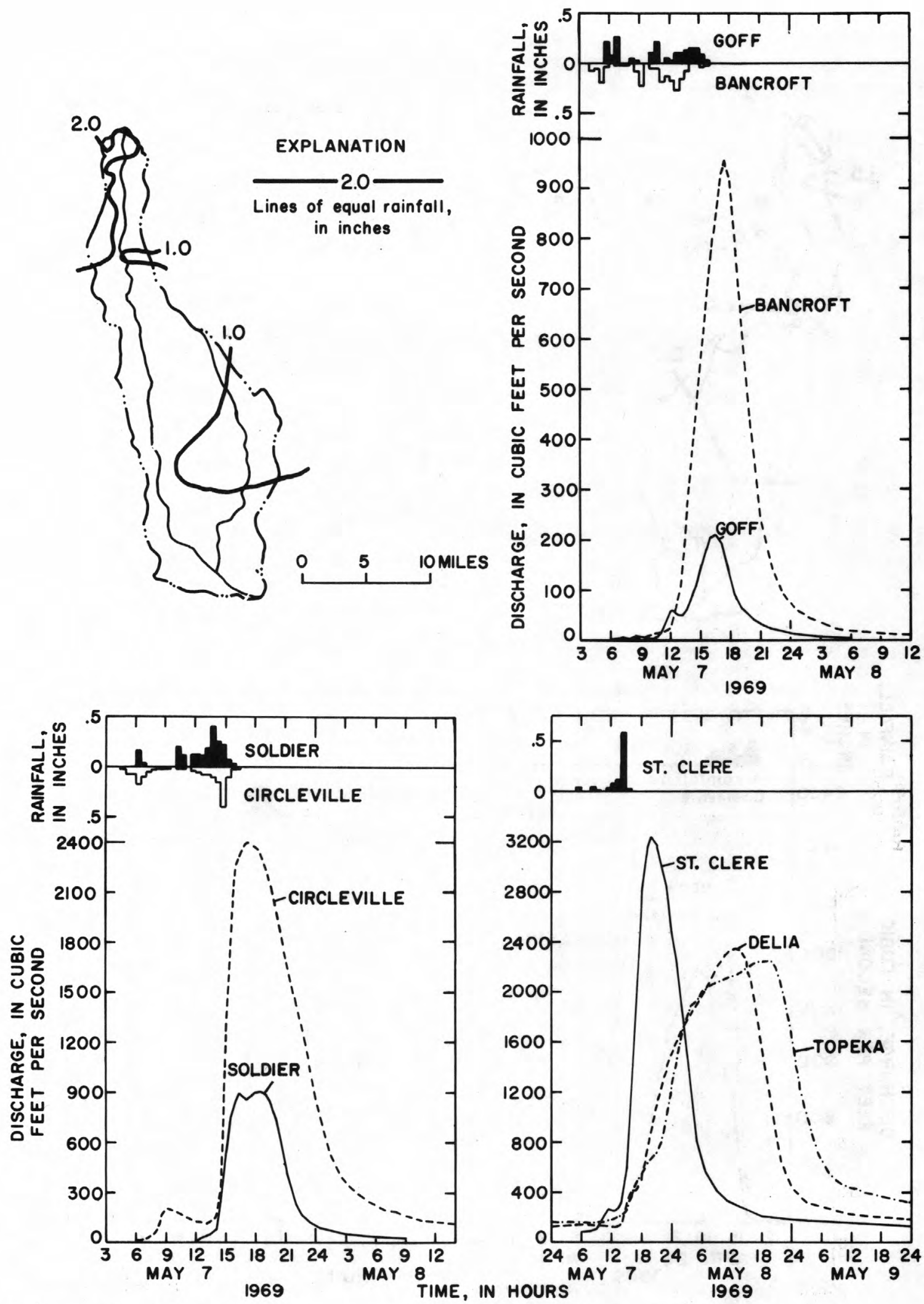

Figure 20.--Distribution of rainfall May 7, 1969, and resulting stream discharge. 

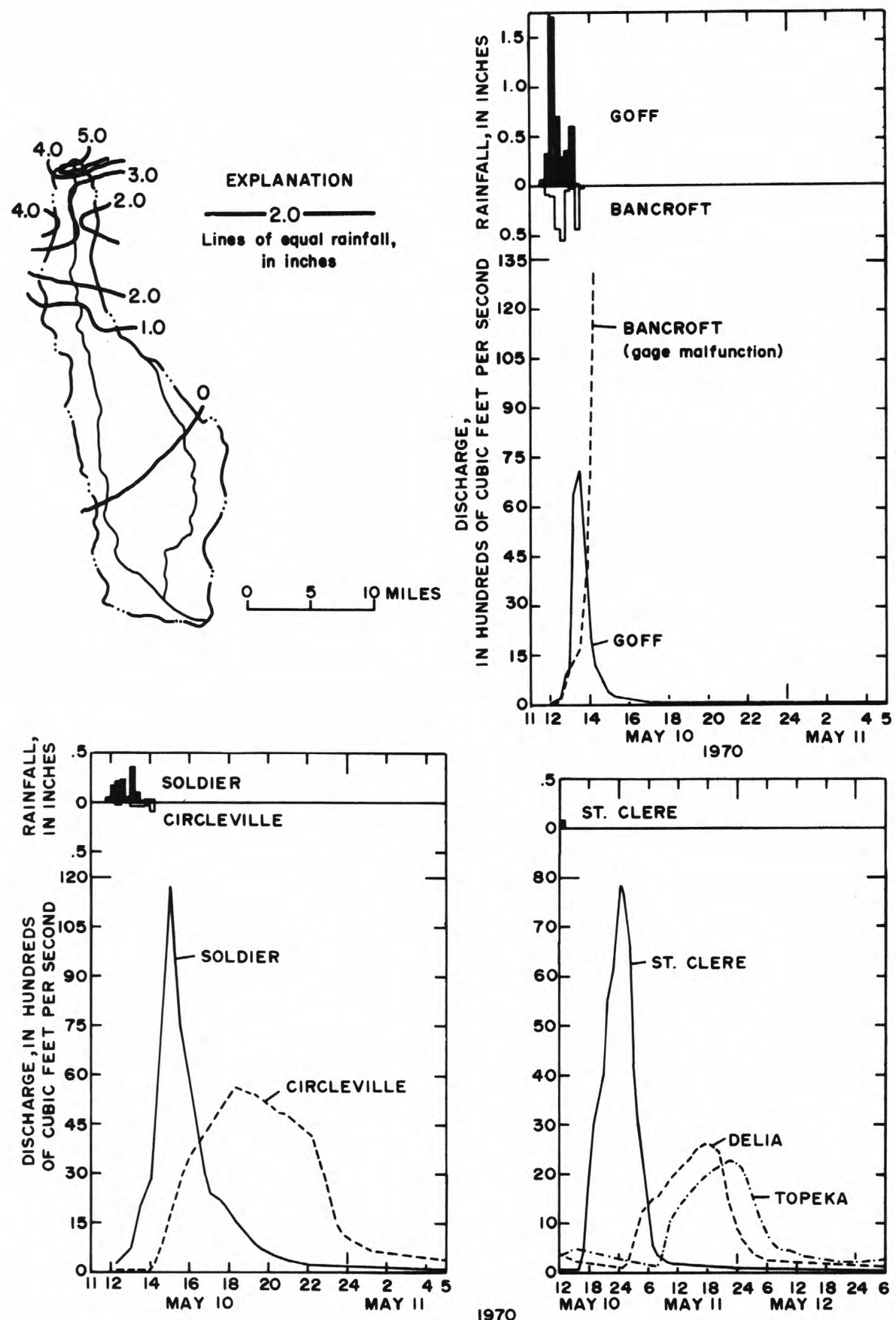

TIME, IN HOURS

Figure 21.--Distribution of rainfall May 10, 1970, and resulting stream discharge. 

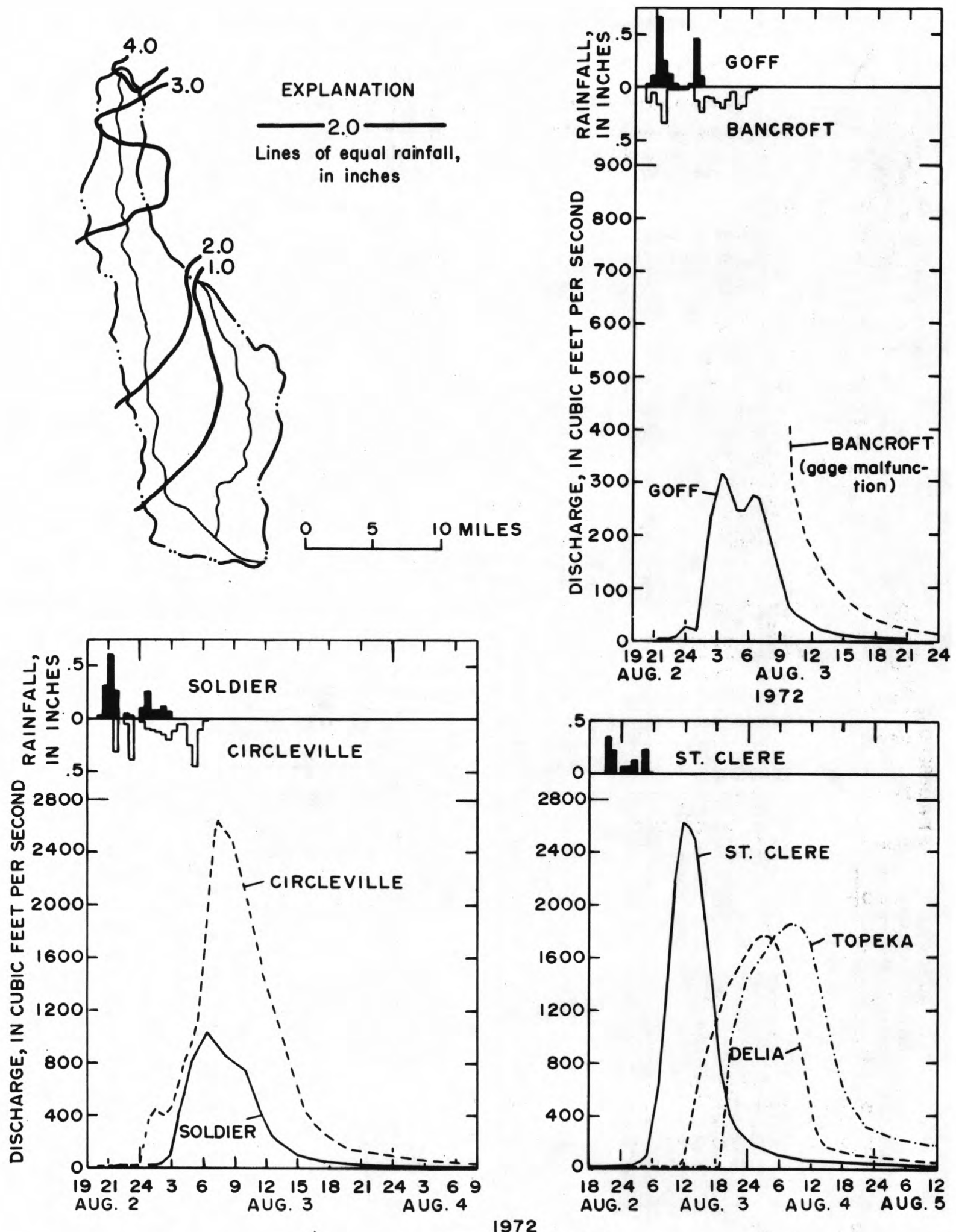

TIME, IN HOURS

Figure 22.--Distribution of rainfall August 2-3, 1972, and resulting stream discharge. 

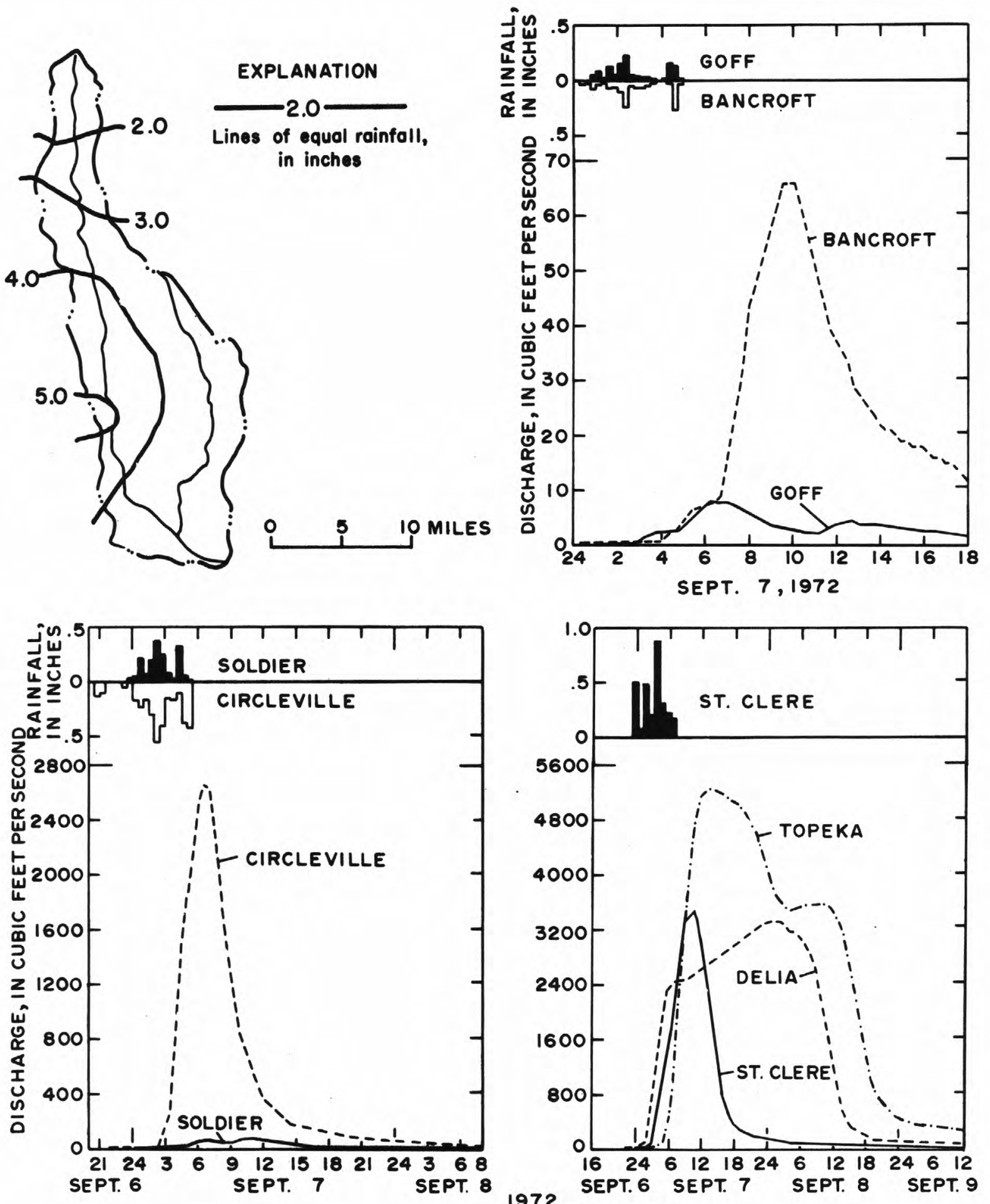

TIME, IN HOURS

Figure 23.--Distribution of rainfall September 6-7, 1972, and resulting stream discharge. 

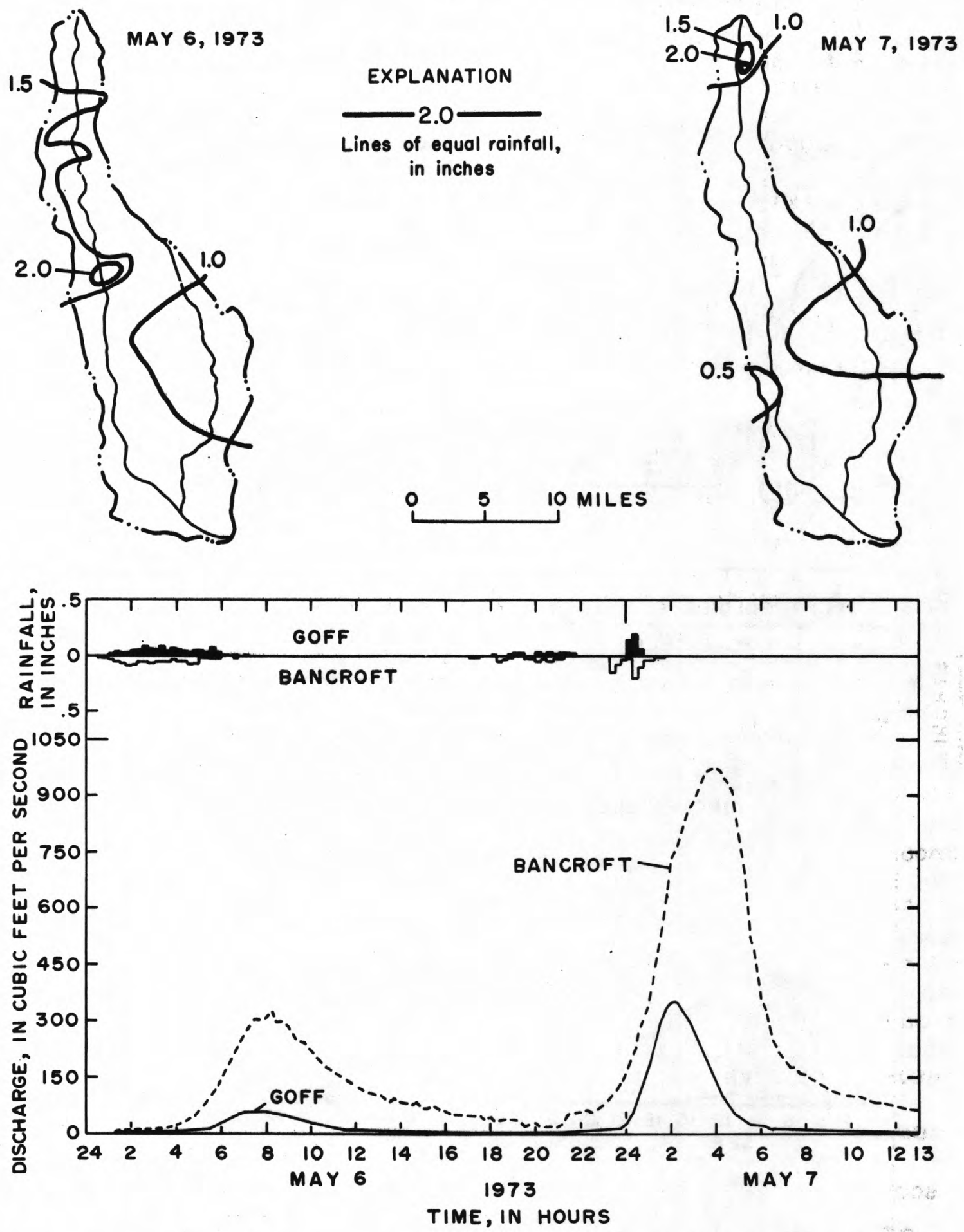

Figure 24.--Distribution of rainfall May 6-7, 1973, and resulting stream discharge. 

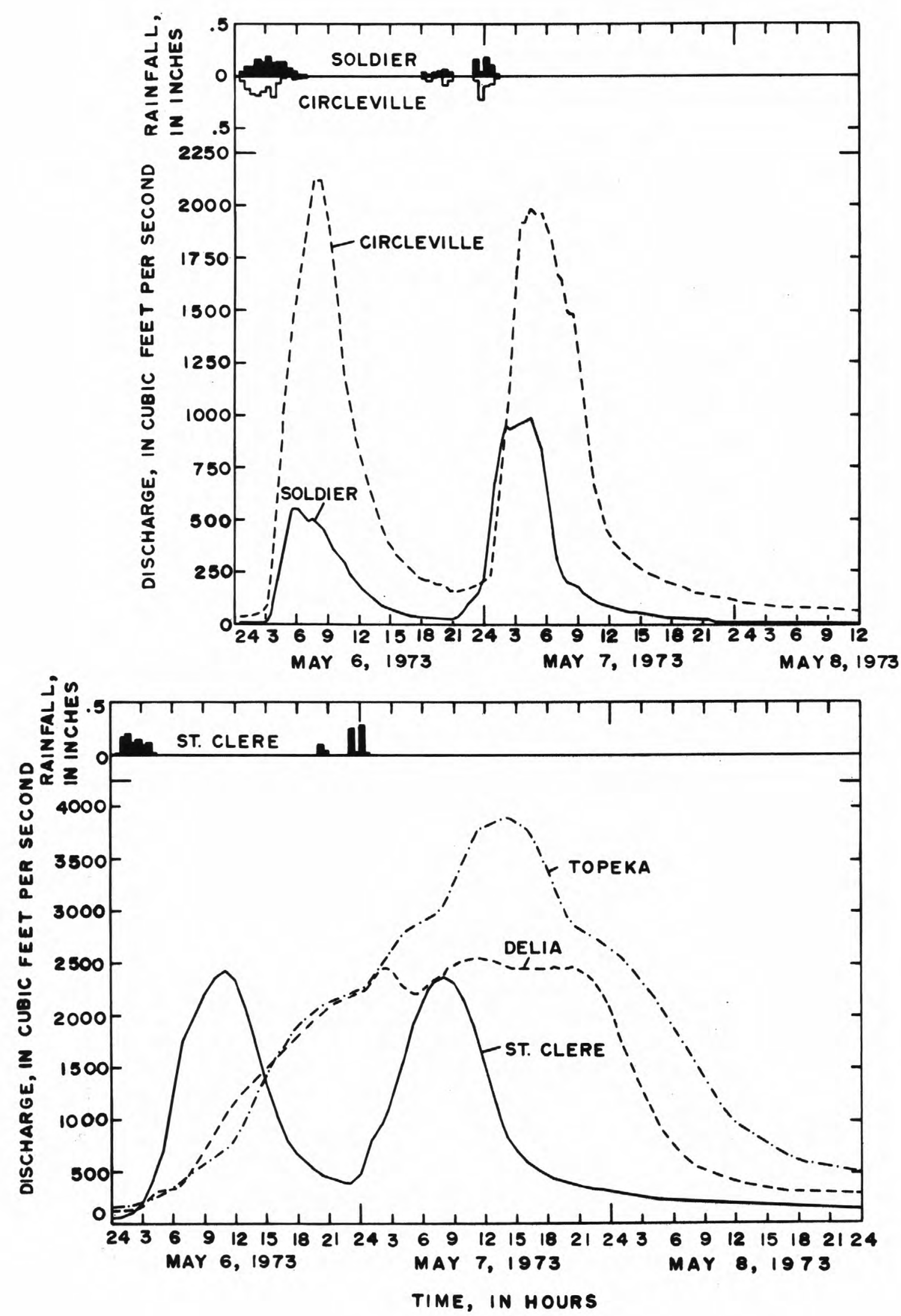

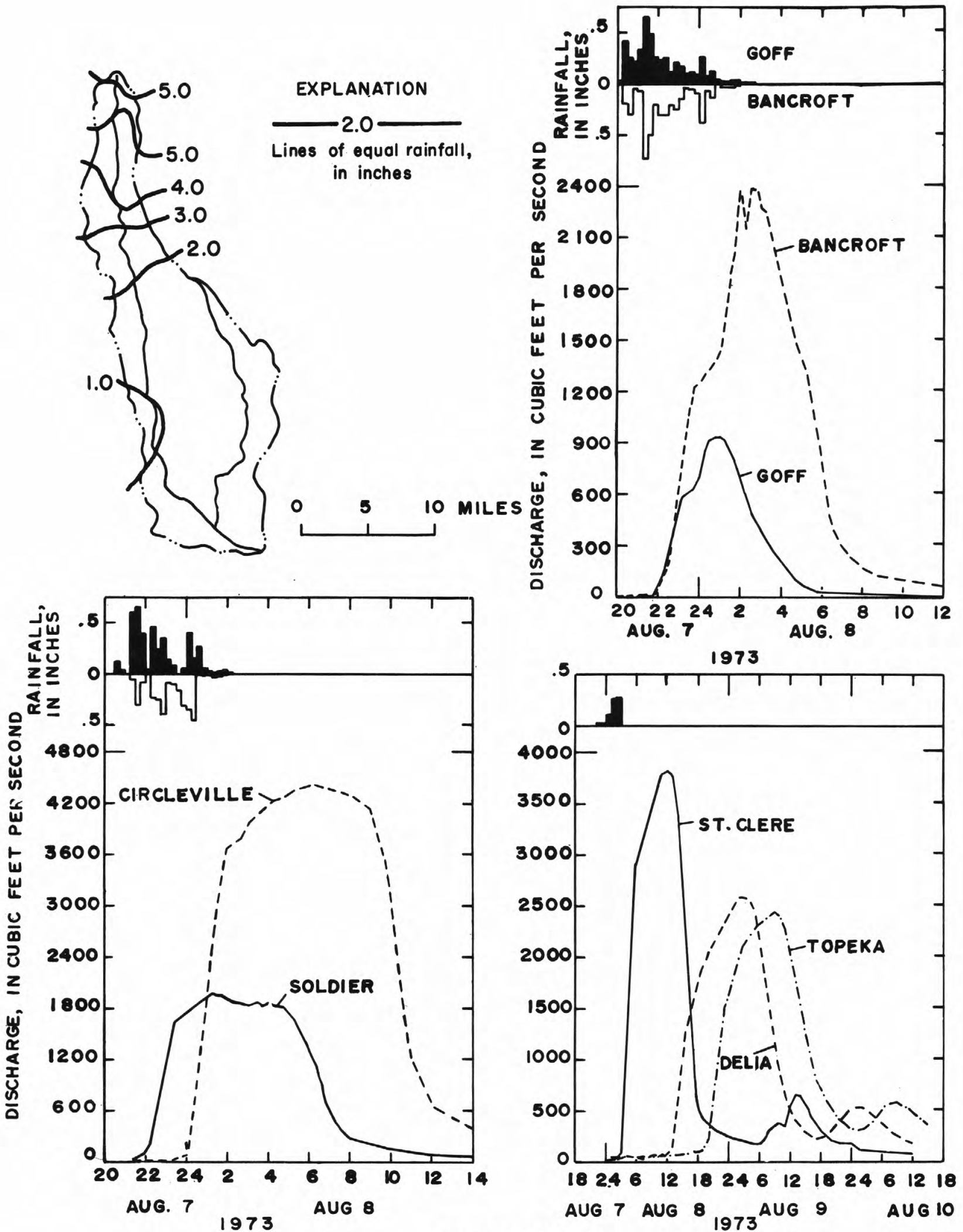

TIME, IN HOURS

Figure 25.--Distribution of rainfall August 7-8, 1973, and resulting stream discharge. 


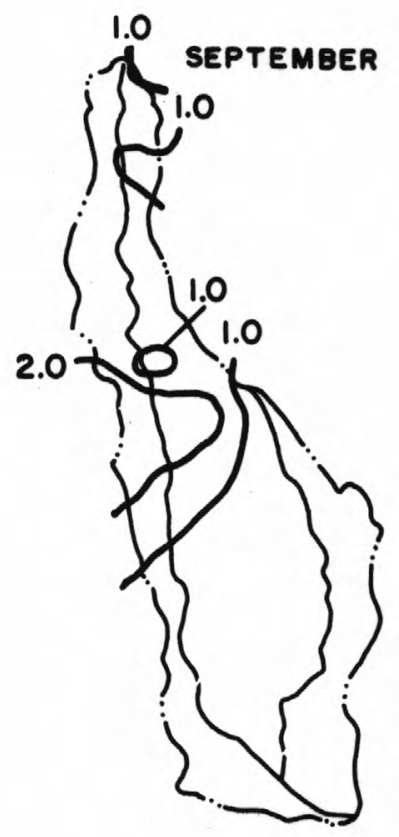

26,1973
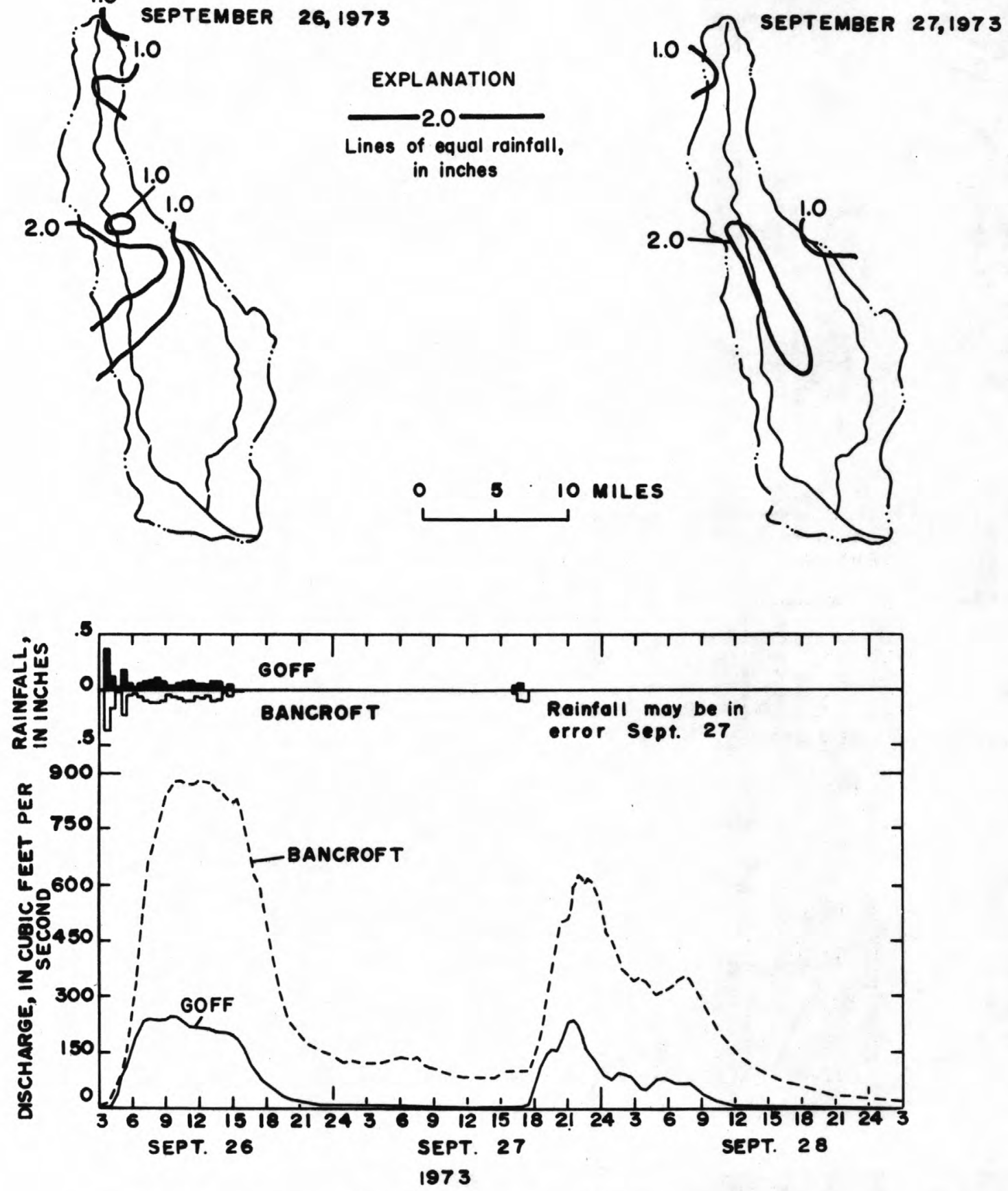

TIME, IN HOURS

Figure 26.--Distribution of rainfall September 26-27, 1973, and resulting stream discharge. 

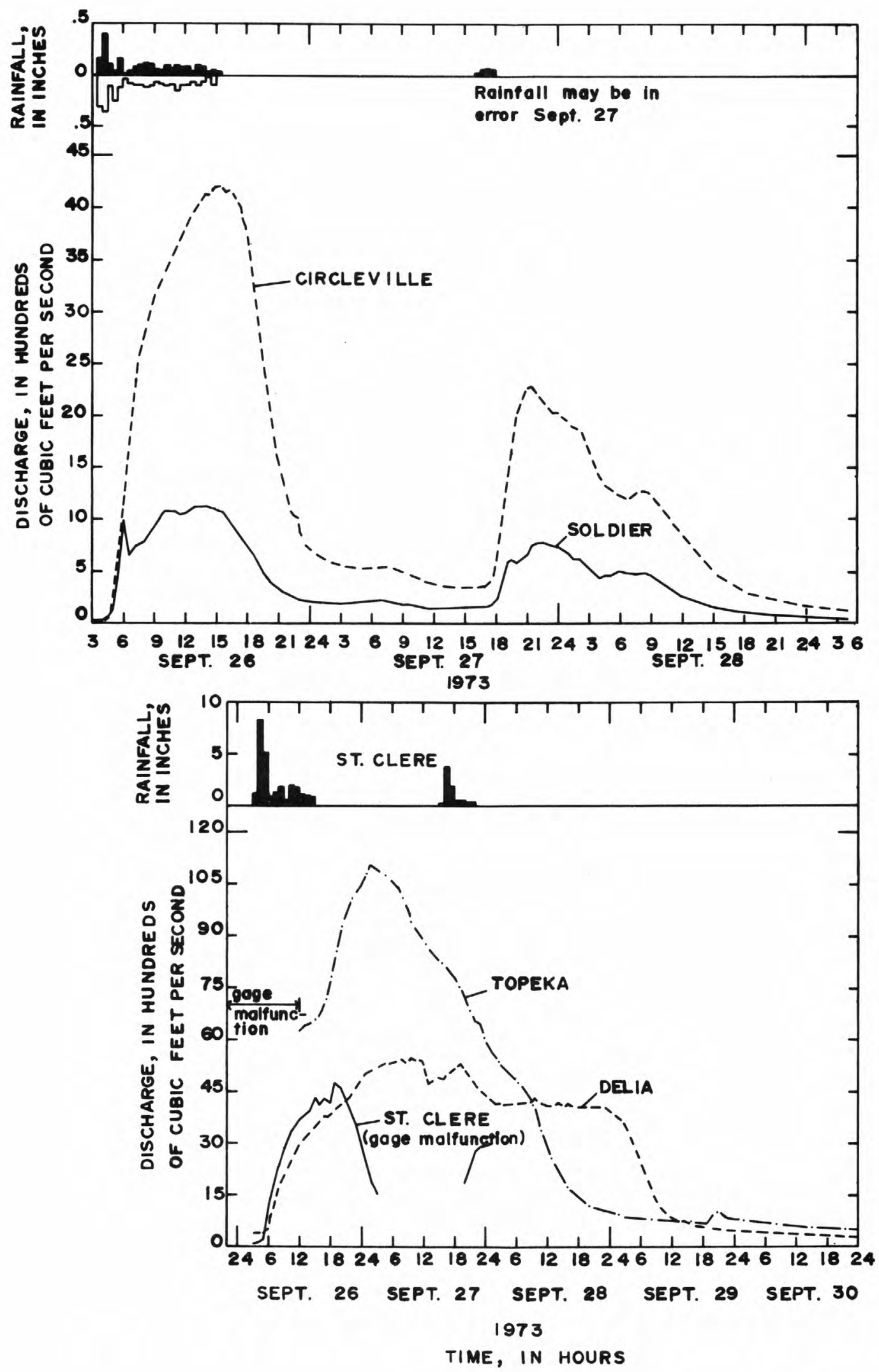

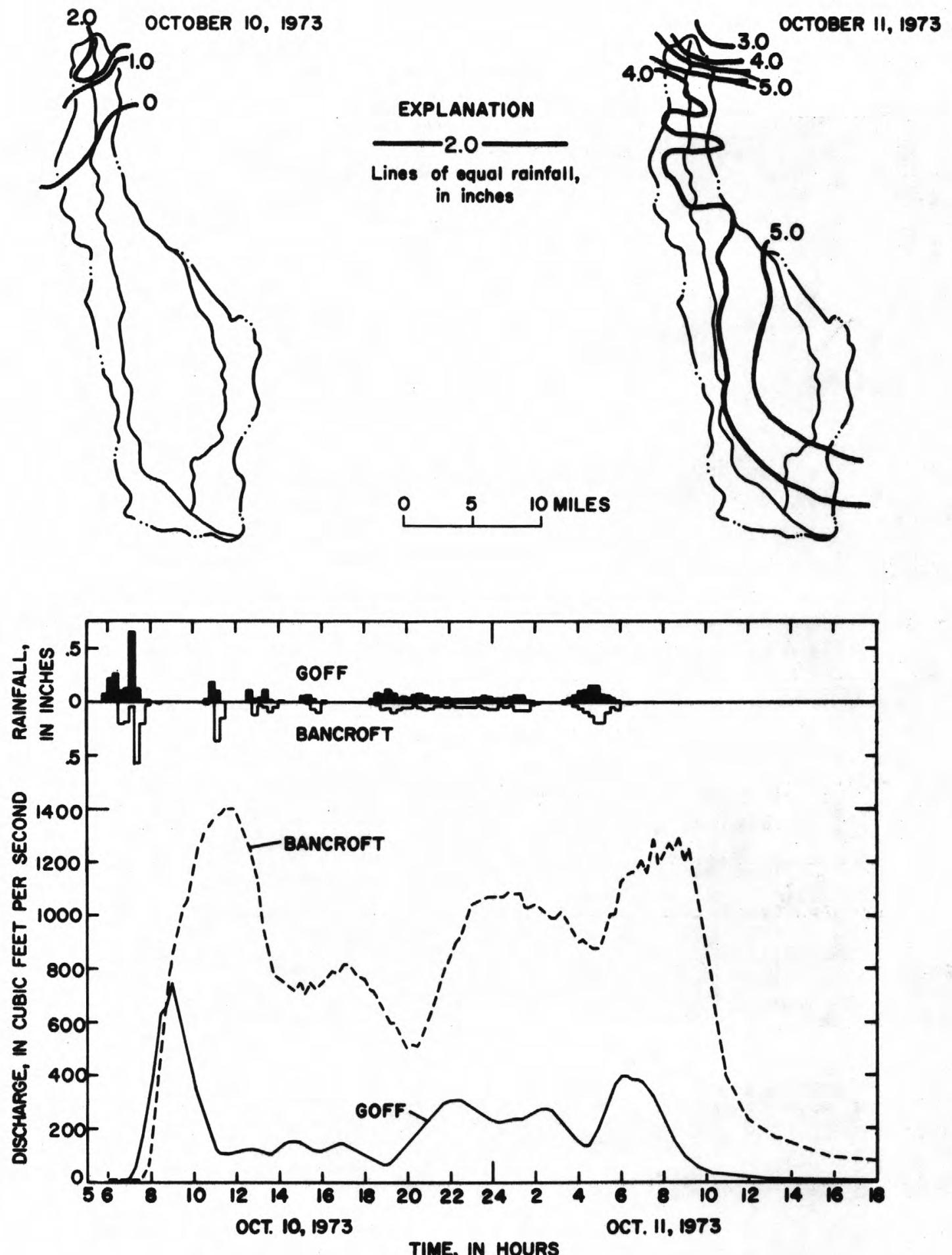

Figure 27.--Distribution of rainfall October 10-11, 1973, and resulting stream discharge. 

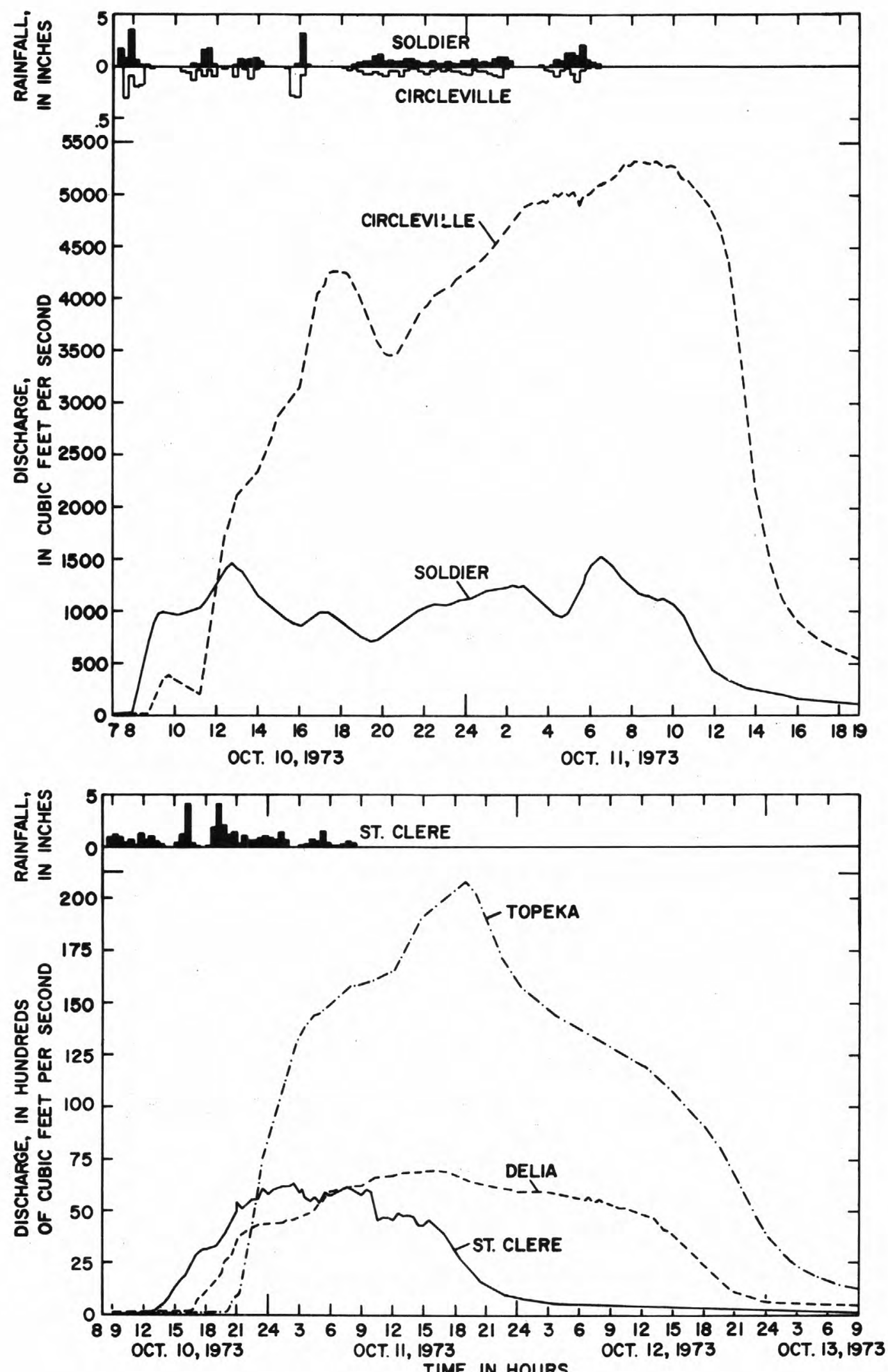

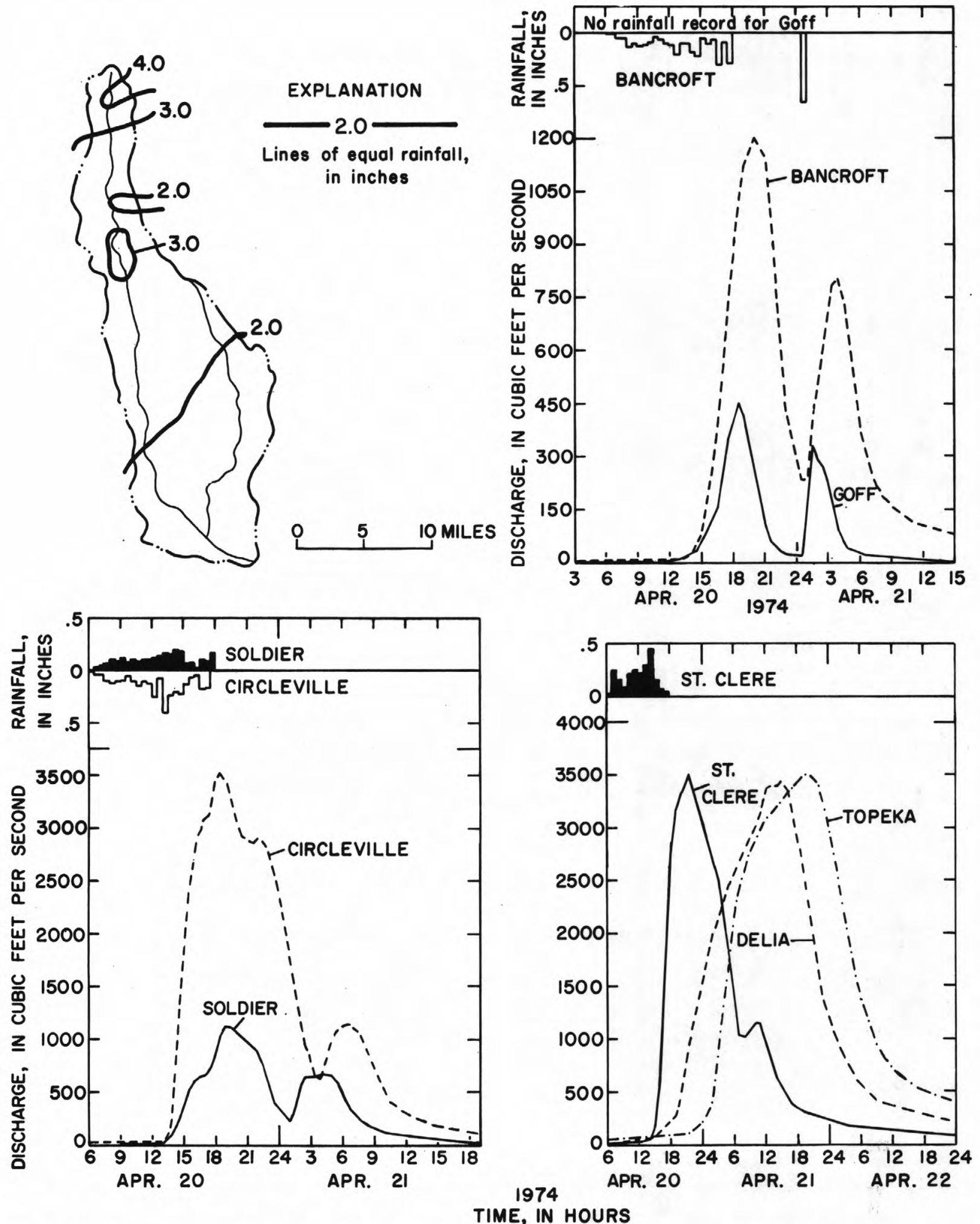

TIME, IN HOURS

Figure 28.--Distribution of rainfall April 20, 1974, and resulting stream discharge. 

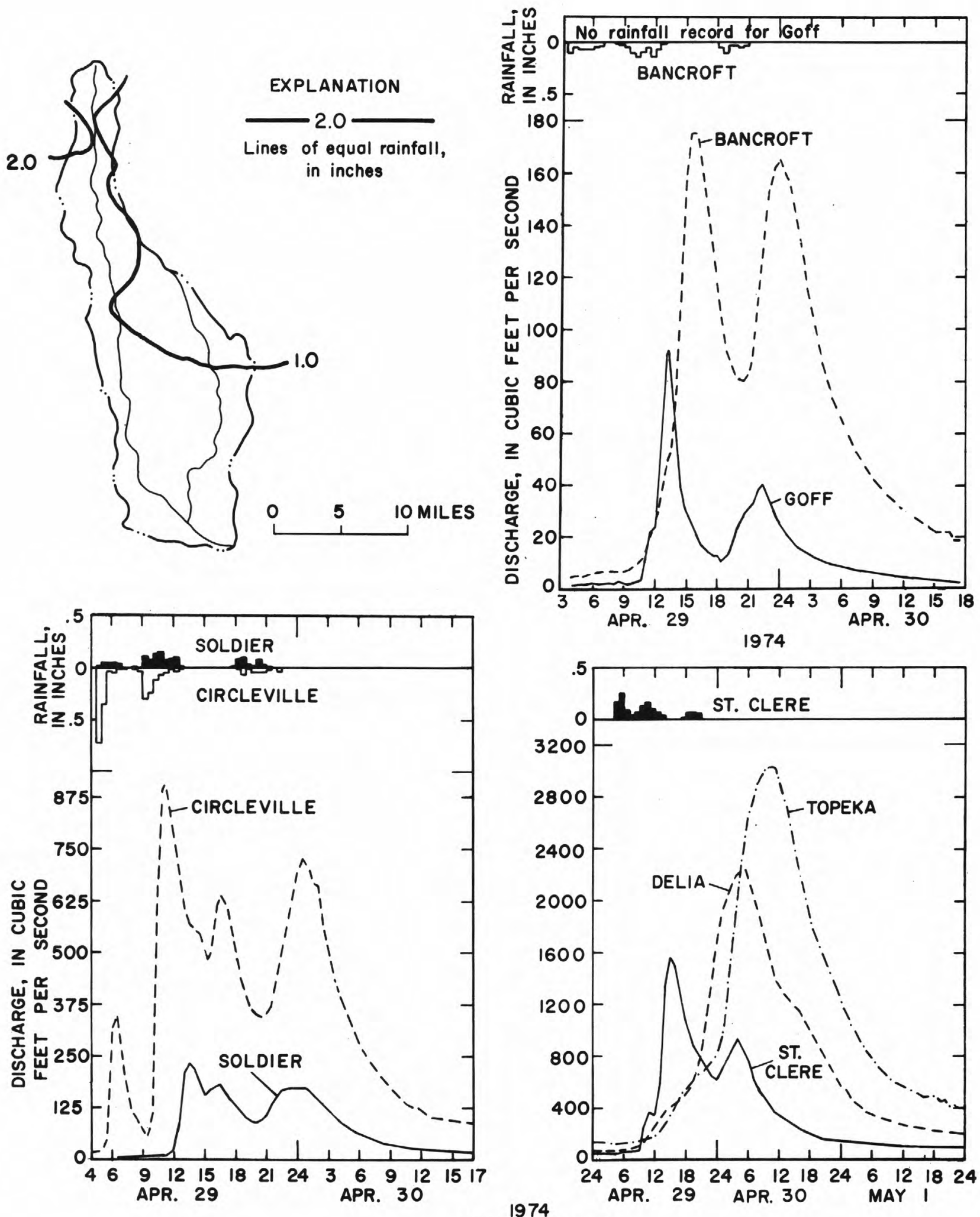

TIME, IN HOURS

Figure 29.--Distribution of rainfall April 29, 1974, and resulting stream discharge. 
Water Quallty

Water in the basin generally may be classed, on the basis of predominant lons, as a calclum-bicarbonate type. A serles of chemical-quality analyses of water from Soldler Creek near Della (November 1967 through August 1968) are listed in table 4. The suspended-sediment data collected in the basin during the 1968 water year are shown in table 5. The data tabulated in tables 4 and 5 are intended to provide sufficlent data for the reader to determine If the Inclusion of water-quallty data from Soldier Creek will be beneficlal to his study.

\section{Seepage-Salinity Investigations}

Main-stem sites included in the investigations are shown in figure 6 . A graphic comparison of discharges measured at these sites $(f / g .30)$ is included only to show the different flow condltions during the investigations. A simllar comparison of dissolved-solids concentrations in water at each site ( $\mathrm{fig}$. 3I) show the difference in chemical quality relative to those flow conditions.

\section{Ground Water}

Water-level fluctuations at the observation-well sites generally reflect streamflow fluctuations. Figure 32 presents hydrographs of water levels in observation well $8-13 \mathrm{E}-1 \mathrm{CCD} 4$ and in Soldler Creek at the St. Clere gaging station for the perlod March through September 1974. The difference in water levels during this perlod generally would indicate the contribution of ground water to streamf low. 
[Chemical Analyses in Milligrams per Liter, 1968 Water Year]

\begin{tabular}{|c|c|c|c|c|c|c|c|c|c|c|c|c|}
\hline DATE & $\begin{array}{l}\text { DIS- } \\
\text { CHARGE } \\
\left(\mathrm{ft}^{3} / \mathrm{s}\right)\end{array}$ & $\begin{array}{l}\text { SILICA } \\
\left(\mathrm{SIO}_{2}\right)\end{array}$ & $\begin{array}{l}\text { DIS- } \\
\text { SOLVED } \\
\text { IRON } \\
(\mathrm{FE})\end{array}$ & $\begin{array}{c}\text { MAN- } \\
\text { GANESE } \\
\text { (MN) }\end{array}$ & $\begin{array}{l}\text { CAL- } \\
\text { CIUM } \\
\text { (CA) }\end{array}$ & $\begin{array}{l}\text { MAG- } \\
\text { NE- } \\
\text { SIUM } \\
\text { (MG) }\end{array}$ & $\begin{array}{c}\text { SODIUM } \\
\text { (NA). }\end{array}$ & $\begin{array}{l}\text { PO- } \\
\text { TAS- } \\
\text { SIUM } \\
(K)\end{array}$ & $\begin{array}{l}\text { BICAR- } \\
\text { BONATE } \\
\left(\mathrm{HCO}_{3}\right)\end{array}$ & $\begin{array}{l}\text { CAR- } \\
\text { BONATE } \\
\left(\mathrm{CO}_{3}\right)\end{array}$ & $\begin{array}{l}\text { SULFATE } \\
\left(\mathrm{SO}_{4}\right)\end{array}$ & $\begin{array}{l}\text { CHLO- } \\
\text { RIDE } \\
\text { (CL) }\end{array}$ \\
\hline $\begin{array}{l}\text { Nov. } \\
02 \ldots \\
22 . .\end{array}$ & $\begin{array}{l}19 \\
15\end{array}$ & $\begin{array}{l}9.8 \\
5.4\end{array}$ & -- & $=$ & $\begin{array}{l}118 \\
114\end{array}$ & $\begin{array}{l}25 \\
29\end{array}$ & $\begin{array}{l}27 \\
25\end{array}$ & $\begin{array}{l}4.8 \\
3.6\end{array}$ & $\begin{array}{l}398 \\
393\end{array}$ & $\begin{array}{l}0 \\
0\end{array}$ & $\begin{array}{l}93 \\
98\end{array}$ & $\begin{array}{l}33 \\
25\end{array}$ \\
\hline $\begin{array}{l}\text { Dec. } \\
23 . . .\end{array}$ & 21 & 6.2 & -- & -- & 118 & 23 & 25 & 2.6 & 381 & 0 & 108 & 20 \\
\hline $\begin{array}{c}\text { Jan. } \\
25 . . .\end{array}$ & 45 & 12 & -- & -- & 72 & 16 & 16 & 3.0 & 254 & 0 & 58 & 11 \\
\hline $\begin{array}{l}\text { Feb. } \\
24 \ldots\end{array}$ & 16 & 6.5 & -- & -- & 107 & 26 & 24 & 1.9 & 356 & 0 & 100 & 23 \\
\hline $\begin{array}{l}\text { Mar. } \\
27 . .\end{array}$ & 11 & 2.2 & -- & -- & 93 & 32 & 25 & 2.6 & 327 & 0 & 105 & 27 \\
\hline $\begin{array}{l}\text { Apr. } \\
18 . . .\end{array}$ & 32 & 13 & .05 & .08 & 85 & 25 & 23 & 3.6 & 303 & 0 & 89 & 15 \\
\hline $\begin{array}{l}\text { May } \\
21 . .\end{array}$ & 16 & 10 & -- & -- & 86 & 18 & 19 & 4.0 & 315 & 0 & 56 & 14 \\
\hline $\begin{array}{c}\text { July } \\
09 . . .\end{array}$ & 3.4 & 12 & -- & -- & 69 & 21 & 29 & 3.6 & 273 & 0 & 62 & 21 \\
\hline $\begin{array}{l}\text { Aug. } \\
10 . . . \\
11 \ldots\end{array}$ & $\begin{array}{l}5560 \\
3270\end{array}$ & $\begin{array}{l}18 \\
29\end{array}$ & $\overline{--}$ & -- & $\begin{array}{l}24 \\
32\end{array}$ & $\begin{array}{l}2.9 \\
3.9\end{array}$ & $\begin{array}{l}4.0 \\
4.8\end{array}$ & $\begin{array}{l}3.6 \\
4.0\end{array}$ & $\begin{array}{r}88 \\
117\end{array}$ & $\begin{array}{l}0 \\
0\end{array}$ & $11^{8.2}$ & $\begin{array}{l}1.0 \\
1.0\end{array}$ \\
\hline DATE & $\begin{array}{l}\text { FLUO- } \\
\text { RIDE } \\
\text { (F) }\end{array}$ & $\begin{array}{c}\text { NITRATE } \\
\left(\mathrm{NO}_{3}\right)\end{array}$ & $\begin{array}{l}\text { PHOS- } \\
\text { PHATE } \\
\left(\mathrm{PO}_{4}\right)\end{array}$ & $\begin{array}{c}\text { BORON } \\
\text { (B) }\end{array}$ & $\begin{array}{l}\text { DIS- } \\
\text { SOLVED } \\
\text { SOLIDS } \\
\text { (RESI- } \\
\text { DUE AT } \\
\left(180^{\circ} \mathrm{C}\right)\end{array}$ & $\begin{array}{l}\text { HARD- } \\
\text { NESS } \\
(C A, M G)\end{array}$ & $\begin{array}{c}\text { NON- } \\
\text { CAR- } \\
\text { BONATE } \\
\text { HARD- } \\
\text { NESS }\end{array}$ & $\begin{array}{c}\text { SODIUM } \\
\text { AD- } \\
\text { SORP- } \\
\text { TION } \\
\text { RATIO }\end{array}$ & $\begin{array}{l}\text { SPECI- } \\
\text { FIC } \\
\text { COND- } \\
\text { UCANCE } \\
\text { (MICRO- } \\
\text { MHOS) }\end{array}$ & PH & & \\
\hline
\end{tabular}

\begin{tabular}{|c|c|c|c|c|c|c|c|c|c|c|}
\hline $\begin{array}{l}\text { Nov. } \\
02 \ldots \\
22 \ldots\end{array}$ & $\begin{array}{l}.3 \\
.3\end{array}$ & $\begin{array}{r}.9 \\
1.3\end{array}$ & $\begin{array}{l}.20 \\
.10\end{array}$ & $\begin{array}{l}.18 \\
.18\end{array}$ & $\begin{array}{l}510 \\
502\end{array}$ & $\begin{array}{l}398 \\
404\end{array}$ & $\begin{array}{l}72 \\
82\end{array}$ & $\begin{array}{l}.6 \\
.5\end{array}$ & $\begin{array}{l}820 \\
780\end{array}$ & $\begin{array}{l}7.6 \\
7.9\end{array}$ \\
\hline $\begin{array}{l}\text { Dec. } \\
23 \ldots\end{array}$ & .3 & 1.3 & .20 & .16 & 500 & 389 & 77 & .6 & 780 & 7.6 \\
\hline $\begin{array}{l}\text { Jan. } \\
25 \ldots\end{array}$ & .3 & 3.1 & .20 & .20 & 315 & 246 & 38 & .4 & 500 & 7.7 \\
\hline $\begin{array}{l}\text { Feb. } \\
24 \ldots\end{array}$ & .2 & .9 & .10 & .10 & 456 & 374 & 82 & .5 & 730 & 7.8 \\
\hline $\begin{array}{c}\text { Mar. } \\
27 . .\end{array}$ & .2 & .4 & .10 & .13 & 472 & 364 & 96 & .6 & 740 & 7.9 \\
\hline $\begin{array}{l}\text { Apr. } \\
18 . . .\end{array}$ & .4 & 3.5 & .20 & .10 & 402 & 375 & 67 & .6 & 660 & 7.7 \\
\hline $\begin{array}{l}\text { May } \\
21 \ldots\end{array}$ & .3 & .9 & .10 & .12 & 378 & 288 & 30 & .5 & 600 & 7.9 \\
\hline $\begin{array}{l}\text { July } \\
09 . .\end{array}$ & .3 & .9 & .20 & -- & 362 & 258 & 34 & .8 & 580 & 8.1 \\
\hline $\begin{array}{c}\text { Aug. } \\
10 . . \\
11 . .\end{array}$ & $\begin{array}{l}.5 \\
.4\end{array}$ & $\begin{array}{l}5.3 \\
4.0\end{array}$ & $\begin{array}{l}1.7 \\
.60\end{array}$ & $\begin{array}{l}.12 \\
.10\end{array}$ & $\begin{array}{l}123 \\
153\end{array}$ & $\begin{array}{l}72 \\
96\end{array}$ & $\begin{array}{l}0 \\
0\end{array}$ & $\begin{array}{l}.2 \\
.2\end{array}$ & $\begin{array}{l}160 \\
210\end{array}$ & $\begin{array}{l}7.1 \\
7.1\end{array}$ \\
\hline
\end{tabular}


Table 5.--Suspended-sediment concentratlons and part|cle-slze distrlbutlon at selected sltes on Soldler Creek durlng 1968 water year.

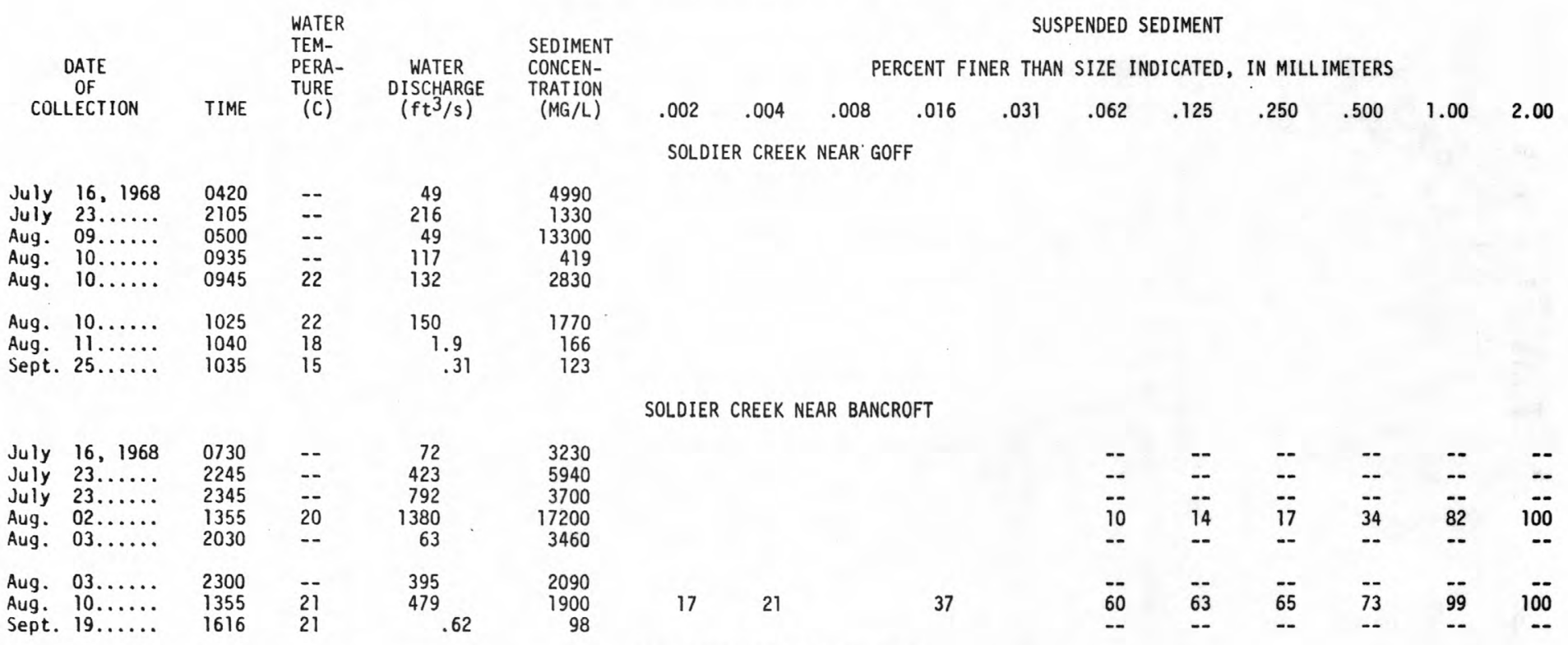

$\begin{array}{llllcr}\text { July } & 23,1968 & 2200 & -- & 242 & 2180 \\ \text { July } 24 \ldots \ldots & 0040 & -- & 740 & 2350 \\ \text { Aug. } 03 \ldots \ldots & 2120 & -- & 328 & 8860 \\ \text { Aug. } 03 \ldots \ldots & 2135 & -- & 750 & 10300 \\ \text { Aug. } 03 \ldots \ldots & 2200 & -- & 1360 & 438 \\ & & & 328 & 398 \\ \text { Aug. } 09 \ldots \ldots & 0740 & -- & 236 & 202 \\ \text { Aug. } 11 \ldots \ldots & 0845 & 18 & 18 & 228 \\ \text { Aug. } 11 \ldots \ldots \ldots & 1325 & -- & 1.1 & 77 \\ \text { Sept. } 19,1968 & 1415 & 22 & & \end{array}$

SOLDIER CREEK NEAR SOLDIER

SOLDIER CREEK NEAR CIRCLEVILLE

$\begin{array}{llllrr}\text { Oct. } 06,1967 & 2345 & -- & 687 & 268 \\ \text { May } 13,1968 & 2200 & -- & 159 & 775 \\ \text { Aug. } 02 \ldots \ldots & 1955 & 21 & 1140 & 5040 \\ \text { Aug. } 09 \ldots \ldots & 0445 & -- & 142 & 3890 \\ \text { Aug. } 09 \ldots \ldots & 0555 & -- & 672 & 3900 \\ & & & & \\ \text { Aug. } 10 \ldots \ldots & 1120 & -- & 1620 & 1500 \\ \text { Sept. } 03 \ldots \ldots & 1900 & -- & 142 & 5280 \\ \text { Sept. } 04 \ldots \ldots & 0020 & -- & 1620 & 9500 \\ \text { Sept. } 04 \ldots \ldots & 1650 & 19 & 100 & 726 \\ \text { Sept. } 19 \ldots \ldots & 1135 & 17 & 6.8 & 30\end{array}$
$18 \quad 24$
45
$79 \quad 83$
84
87
93
100

SOLDIER CREEK NEAR ST. CLERE

$\begin{array}{llllrr}\text { Oct. } 07,1967 & 0100 & -- & 588 & 2260 \\ \text { Oct. } 07 \ldots \ldots \ldots & 0205 & -- & 1110 & 7180 \\ \text { July } 30,1968 & 0920 & -- & 1110 & 2840 \\ \text { July } 30 \ldots \ldots & 1150 & -- & 1800 & 501 \\ \text { July } 31 \ldots \ldots & 1155 & -- & 3110 & 1940 \\ & & & \\ \text { Sept. } 03 \ldots \ldots & 2320 & -- & 680 & 6670 \\ \text { Sept. } 04 \ldots \ldots & 0535 & -- & 1860 & 5110 \\ \text { Sept. } 04 \ldots \ldots & 1450 & 19 & 321 & 2000 \\ \text { Sept. } 18 \ldots \ldots & 1600 & 19 & 22 & 56\end{array}$

$\begin{array}{ll}-- & -- \\ -- & -- \\ -- & - \\ -- & - \\ -- & - \\ 22 & 30 \\ 26 & 36 \\ 20 & 36 \\ -- & --\end{array}$

$\begin{array}{lrr}-- & -- & \\ -- & -- & \\ -- & -- & \\ -- & -- & \\ 57 & 100 & \\ 62 & 99 & 100 \\ 74 & 100 & \\ -- & -- & \end{array}$

SOLDIER CREEK NEAR DELIA

\begin{tabular}{|c|c|c|c|c|c|c|c|c|c|}
\hline \multirow{5}{*}{$\begin{array}{l}\text { Oct. } \\
\text { Oct. } \\
\text { Apr. } \\
\text { Apr. } \\
\text { July }\end{array}$} & 07,1967 & 1300 & -- & 1000 & 4310 & -- & -- & -- & -- \\
\hline & $07 \ldots \ldots$ & 1420 & -- & 1220 & 1340 & -- & -- & $=$ & \\
\hline & 16,1968 & 0830 & -- & 1000 & 13800 & 36 & 45 & 77 & 100 \\
\hline & $16 \ldots \ldots$ & 0855 & -- & 1220 & 12700 & 36 & 47 & 75 & 100 \\
\hline & $24 \ldots \ldots$ & 0340 & & 881 & 3710 & - & -- & -- & -- \\
\hline July & & 0420 & -- & 1120 & 60600 & -- & -- & -- & - \\
\hline July & $24 \ldots$ & 0605 & -- & 1440 & 84100 & -- & -- & -- & \\
\hline Sept. & $17 .$. & 1430 & 18 & 28 & 131 & -- & -. & -- & - \\
\hline
\end{tabular}




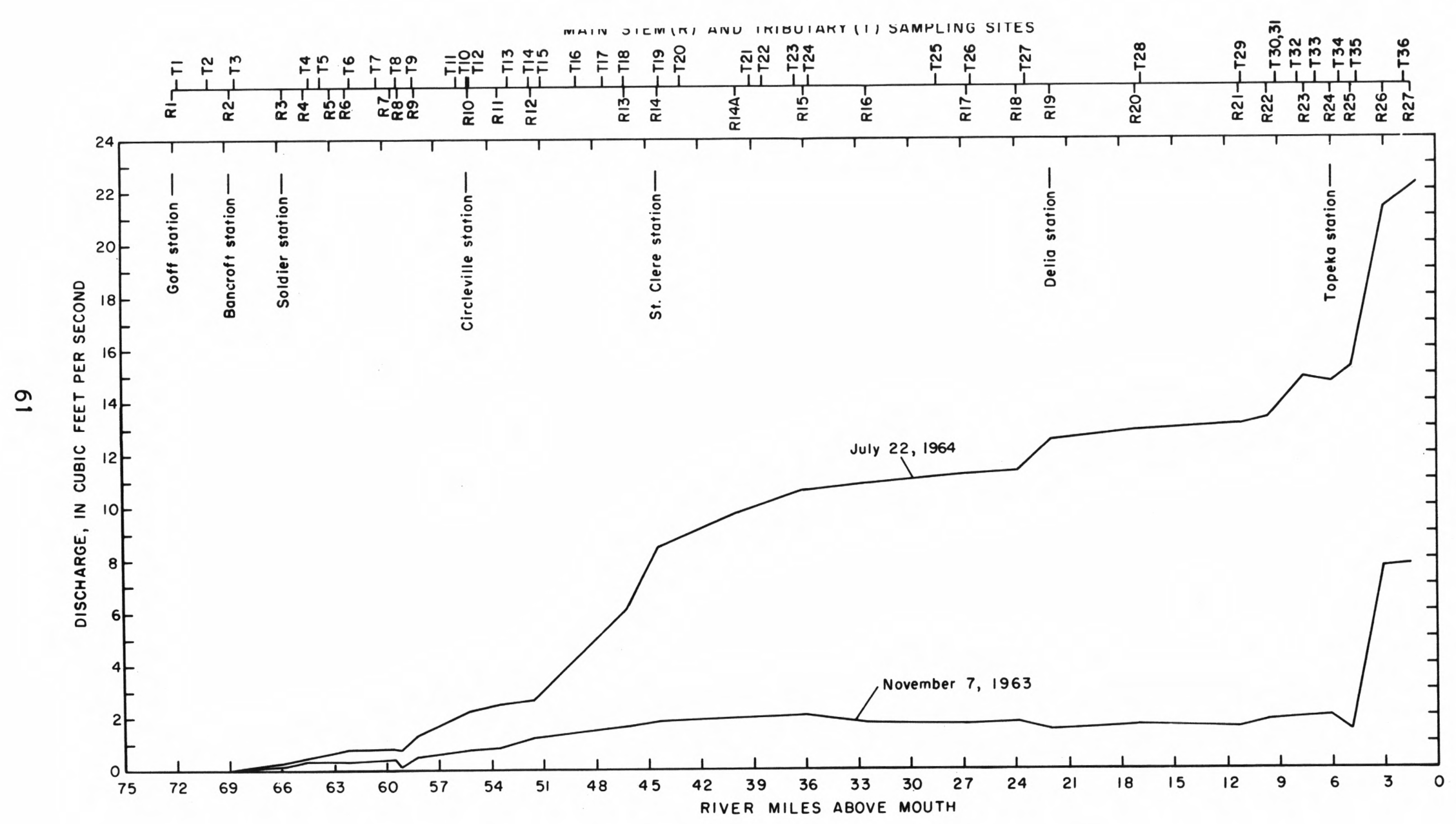

Figure 30.--Water discharges at main-stem sites along Soldier Creek. 


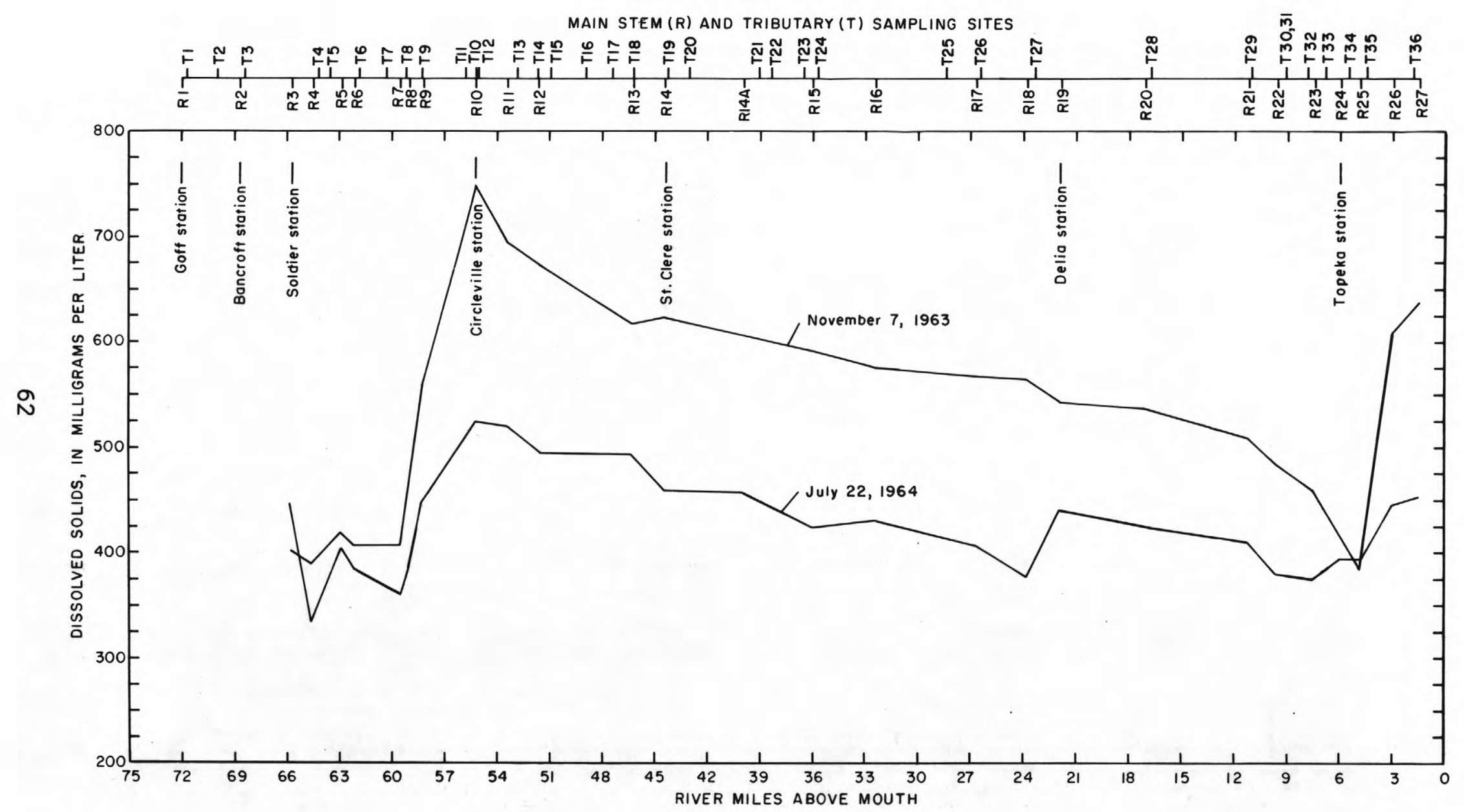

Figure 31.--Dissolved-sollds concentrations at main-stem sites along Soldier Creek. 


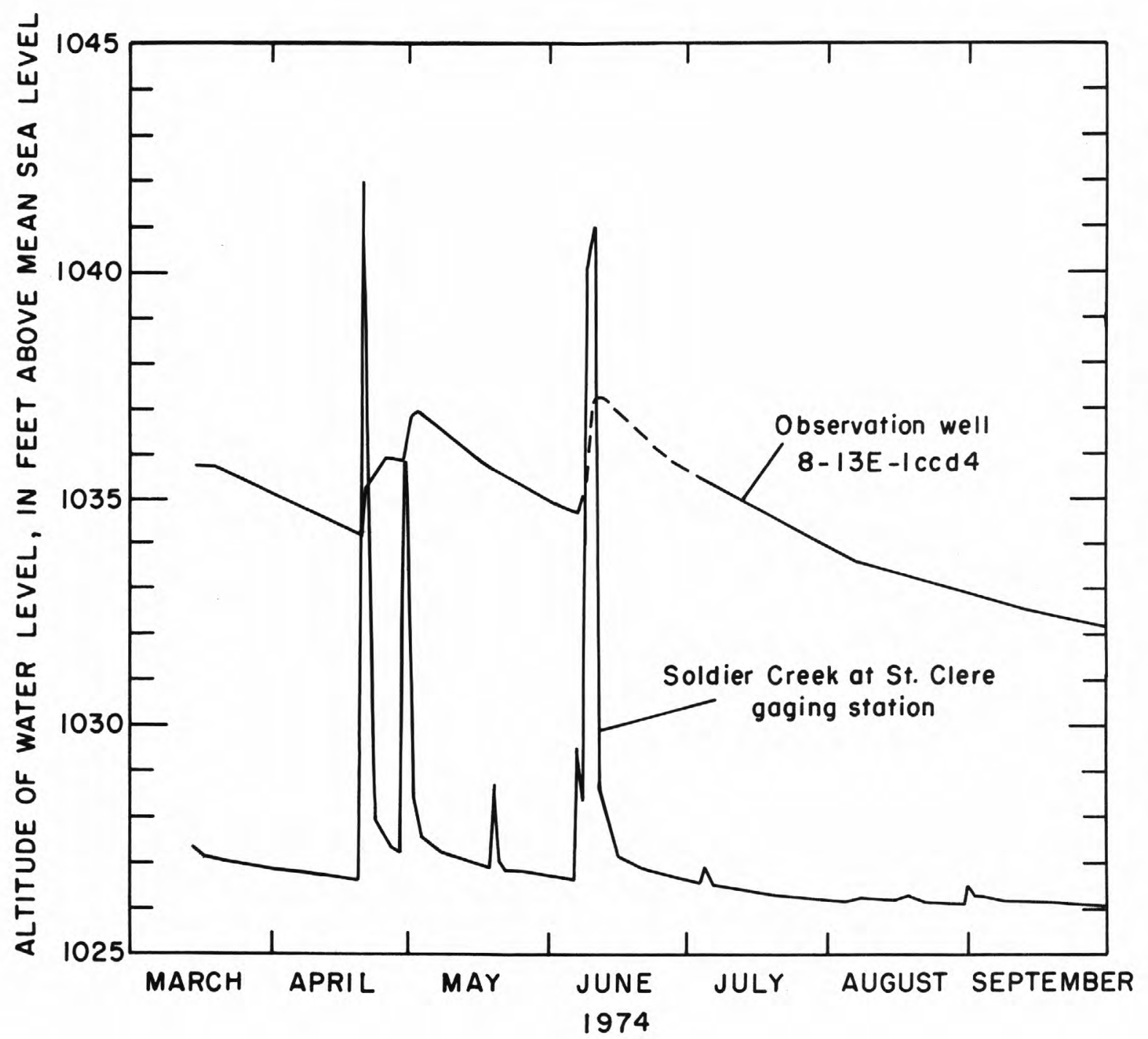

Figure 32.--Hydrographs of water levels in observation well 8-13E-1CCD4 and in Soldier Creek near St. Clere gaging station. 
Soldler Creek basin is a long, narrow basin encompassing an area of about 290 square miles almost directly north of Topeka, Kansas. A wide range of hydrologic data has been collected in the basin since the spring of 1964. These data include rainfall, stream discharge, sediment concentrations, chemlcal quality of water, and ground-water altitudes.

The data collection system conslsts of 7 recording streamflow stations, 5 recording rainfall stations, 51 nonrecording ralnfall stations, and 31 groundwater observation wells. Sediment and chemical quality of water samples were collected intermittently at selected sites.

A synopsis of the time and space distribution of rainfall and peak flow are provided in graphlc and tabular form for selected events of rainfall and peak flow. Peak discharges assoclated with graphic presentations of rainfall and resulfing hydrographs range from $7,080 \mathrm{ft}^{5} / \mathrm{s}$ at the smallest dralnage to $20,800 \mathrm{ft} / \mathrm{s}$ at the largest drainage. Representative data concerning the chemlcal quality of water and the fluvial sediment al so are Included. Selected ground-water and seepage-investigation data are depicted graphically.

The data-collection system and a synopsis of the data which it produced are discussed in sufficient detall to permit a potentlal user to declde if the data wlll fit his needs. These data will provide a wealth of information for a wide range of hydrologic studies.

\section{SELECTED REFERENCES}

Abmeyer, Walter, and Campbell, C. V., 1970, Soll survey of Shawnee County, Kansas: U.S. Department of Agriculture, Soll Conservation Service, 77 p.

Benson, M. A., 1962, Factors influencing the occurrence of floods in a hum!d reglon of diverse terrain: U.S. Geol. Survey Water-Supply Paper 1580-B, $64 \mathrm{p}$.

Carswell, W. J., Jr., Hydrologlc data for Sold?er Creek bas In, Kansas: (In preparation).

Flora, S. D., 1948, Climate of Kansas: Kansas State Board of Agrlculture Rept., v. 67, no. 285,320 p.

Hershfleld, D. M., 1961, Rainfall frequency atlas of the United States: U.S. Weather Bureau Tech. Paper No. 40, 115 p.

U.S. Geological Survey, Water resources data for Kansas: Water-Data Reports, 1964-76.

Walters, K. L., 1953, Geology and ground-water resources of Jackson County, Kansas: Kansas Geol. Survey Bull. 101, 90 p.

Ward, John R., 1974, Geohydrology of Nemaha County, northeastern Kansas: Kansas Geol. Survey Ground Water Serles No. 2, 19 p. 


\section{APPENDIX A}

\section{Definition of Terms for Basin Characteristics}

Maln-channel slope - In feet per mile, computed by the 85- to 10-percent method described by Benson (1962).

Slope at site - In feet per mile, is the stream slope computed from two or more contour crossings from 1: 24,000 scale topographlc maps.

Stream length - In miles, measured along channel from gage to basin divide.

Valley length - In miles, measured along general path of flood plain from gage to basin divide.

Mean basin elevation - In feet above mean sea level, measured from topographic maps by transparent grid sampling method (40 to 80 points in the basin were sampled).

Basin perimeter - In miles, is the length of divide around the drainage basin above the site, as measured on 1: 250,000 scale maps.

Storage - Area of lakes, ponds, and swamps in percent of contributing drainage area, measured by the grid sampling method.

Forest - Forested area in percent of contrlbuting drainage area, measured by grid sampling method.

Mean annual precipitation - In inches, at centroid of basin, for period 194i-70. Obtained from written communication by L. D. Bark, Kansas Agricultural Experiment Station, Manhattan, Kansas.

I 24,2 - Precipitation intensity for 24-hour rainfall, in inches, expected at centroid of basin on the average of once each 2 years. Estimated from U.S. Weather Bureau Technical Paper 40.

I 24,50 - Precipitation intensity for 24-hour rainfall, in inches, expected at centrold of basin on the average of once each 50 years. Estimated from U.S. Weather Bureau Technical Paper 40.

\section{Streamflow Station Descriptions and Selected Basin Characteristics}

\section{Soldier Creek near Goff, KS}

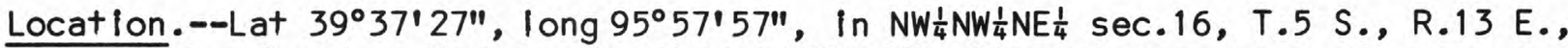
Nemaha County, Hydrologic Unit 10270102, 20 feet downstream from county road, $3.3 \mathrm{miles}$ southwest of Goff, and at river mile 71.9.

Drainage area.--2.06 square miles, as measured on U.S. Geological Survey $7 \frac{1}{2}-m i n u t e$ quadrangle sheets.

Establishment.--March 1, 1964, by U.S. Geological Survey, Kansas District.

Gage.--Digital-punch recorder, 15-minute interval, driven by 50-foot-range bubble gage in $4 \times 4 \times 8-f o o t$ steel-covered shelter on left bank at downstream side of bridge. Auxiliary equipment includes a graphical recorder.

Datum of gage is 1,297.10 feet above NGVD of 1929 . 
History.--Nov. 19, 1963 - Graphical recorder was started In operation. Gageheight record only collected through the winter months.

March 1,1964-Regular gaging station established as part of a small streams investigation project.

Channel and control.--The channel near the gage is cut in glaclal till. The low-water control is a riffle on glaclal sand and gravel 10 feet downstream from the orifice. The high-water control is normally at a sharp bend 150 feet downstream from the bridge. The stream traverses a heavlly timbered area near the gage. Right-bank overflow starts at about 9 feet and left-bank overflow at about 13 feet.

Historic Floods. --No flood information avallable prlor to establishment of gage. Regulation and diversion.--No Irrigation or diversion upstream is known.

Maps.--Station is located on the Goff, Kansas, U.S. Geological Survey $7 \frac{1}{2}-m i n u t e$ quadrangle, topographic map.

\section{Selected climatic and physlographic basin parameters.--}

$\begin{array}{lc}\text { Main-channel slope } & 25.1 \mathrm{ft} / \mathrm{ml} \\ \text { Slope at site } & 29.3 \mathrm{ft} / \mathrm{mi} \\ \text { Stream length } & 2.94 \mathrm{ml} \\ \text { Valley length } & 2.67 \mathrm{mi} \\ \text { Mean basin elevation } & 1,360 \mathrm{ft} \\ \text { Basin perimeter } & 6 \mathrm{ml} \\ \text { Storage } & 0.2 \text { percent } \\ \text { Forest } & 1.7 \text { percent } \\ \text { Mean annual preclpitation } & 34.8 \mathrm{in} \\ \text { I } 24,2 & 3.30 \mathrm{in} \\ \text { I } 24,50 & 6.55 \mathrm{in}\end{array}$

06889120 Soldier Creek near Bancroft, KS

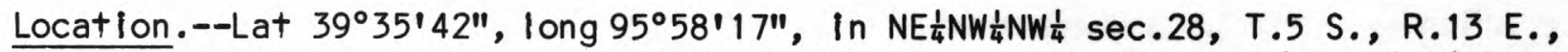
Nemaha County, Hydrologlc Unit 10270102, at downstream side of highway bridge, 4.0 miles west of Bancroft, and at river mlle 68.7.

Drainage area. --10.5 square miles, all but 0.7 square $\mathrm{mlles}$ of which was measured on U.S. Geological Survey $7 \frac{1}{2}-m i n u t e$ quadrangle sheets.

Establishment.--March 1, 1964, U.S. Geological Survey, Kansas District.

Gage.--Digital-punch recorder, 15-minute Interval, driven by 50-foot-range bubble gage in $4 \times 4 \times 8$-foot steel-covered shelter on right bank at downstream side of bridge. Auxlliary equipment includes a graphical recorder.

Datum of gage is 1,239.50 feet above NGVD of 1929 . 
History.--Nov. 19, 1963 - Graphical recorder was started in operation. Gagehelght record only collected through the winter months.

March 1,1964 - Regular gaging station established as part of a small streams investigation project.

Channel and control. - The channel near the gage is alluvium about 2 feet thick over bedrock and is not stable. The low-water control is usually a sand riffle at different locations within about 50 feet of the gage. Both banks are heavlly wooded. Right-bank overflow begins at about 9.5 feet and left-bank overflow at about 11 feet.

Historic Floods.--Francis Swartz (rainfall observer, first house east on north side of road) states that before the road and bridge were ralsed (about 1961), water was often over the road.

Regulation and diversion.--No irrigation is known above the station. Gravel quarry operation in SW $\frac{1}{4}$ sec.5, T.5 S., R.13 E., at times may affect flow at gage. This is commonly accompanied by a rust color in the water. Beavers are known to frequent the area.

Maps.--Station is located on Soldier, Kansas, U.S. Geological Survey $7 \frac{1}{2}-m i n u t e$ quadrangle, topographic map.

Selected climatic and physlographic basin parameters.--

Main-channel slope
Slope at site
Stream length
Valley length
Mean basin elevation
Basin perimeter
Storage
Forest
Mean annual precipltation
I 24,2
I 24,50

Main-channel slope

Slope at site

Stream length

Valley length

Mean basin elevation

Basin perimeter

Storage

Forest

I 24,2

I 24,50

\author{
$18.0 \mathrm{ft} / \mathrm{ml}$ \\ $8.1 \mathrm{ft} / \mathrm{mi}$ \\ $6.24 \mathrm{mi}$ \\ $4.96 \mathrm{mi}$ \\ $1,350 \mathrm{ft}$ \\ $9 \mathrm{mi}$ \\ 0.2 percent \\ 5.8 percent \\ 34.9 in \\ $3.30 \mathrm{in}$ \\ $6.55 \mathrm{in}$
}

06889140 Soldier Creek near Soldier, KS

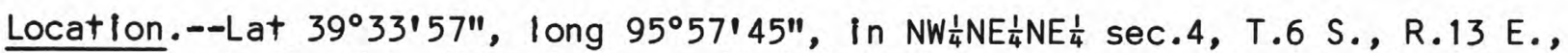
Jackson County, Hydrologic Unit 10270102, at downstream side of highway bridge, $2.0 \mathrm{ml}$ les north of Soldier and at river mile 65.7.

Drainage area. --16.9 square miles, all but 0.7 square miles of which was measured on U.S. Geological Survey $7 \frac{1}{2}$-minute quadrangle sheets.

Establishment.--March 1, 1964, by U.S. Geologlcal Survey, Kansas Distrlct. 
Gage.--Digital-punch recorder, 15-minute interval, driven by a 50-foot-range bubble gage in a $4 \times 4 \times 8-$ foot steel-covered shelter on left downstream side of bridge. Auxillary equipment includes a graphical recorder. Datum of gage is 1,206.00 feet above NGVD of 1929 .

History.--Nov. 19, 1963 - Graphlcal recorder was started in operation. Gageheight record only collected through the winter months. March 1,1964-Regular gaging station established as part of a small streams investigation project.

Channel and control. --The channel near the gage is a thin covering of alluvium over bedrock and is not stable. The bridge at the gage is of recent construction, and considerable scouring and shifting of the channel can be expected until it reaches equilibrium. The low-water control is usually a sand-gravel riffle at different locations within 200 feet of the gage. Both banks are heavlly wooded. Right-bank overflow begins at about 12 feet and is about 300 feet wide. Left-bank overflow begins at about 13 feet and may be as much as 1,000 feet wide.

Historic Floods. --No flood information avallable prior to establishment of gage.

Regulation and diversion.--No irrigation is known above the station. Beavers are known to frequent the area and may cause some regulation during low-flow perlods.

Maps.--Station is located on Soldier, Kansas, U.S. Geological Survey $7 \frac{1}{2}-m i n u t e$ quadrangle, topographlc map.

Selected climatic and physlographic basin parameters.--

$\begin{array}{lc}\text { Main-channel slope } & 14.6 \mathrm{ft} / \mathrm{mi} \\ \text { Slope at site } & 12.0 \mathrm{ft} / \mathrm{ml} \\ \text { Stream length } & 9.29 \mathrm{ml} \\ \text { Valley length } & 7.33 \mathrm{ml} \\ \text { Mean basin elevation } & 1,340 \mathrm{ft} \\ \text { Basin perimeter } & 16 \mathrm{ml} \\ \text { Storage } & 0.2 \text { percent } \\ \text { Forest } & 7.0 \text { percent } \\ \text { Mean annual precipitation } & 34.9 \mathrm{in} \\ \text { I 24,2 } & 3.32 \mathrm{in} \\ \text { I 24,50 } & 6.57 \mathrm{in}\end{array}$

06889160 Soldier Creek near Circleville, KS

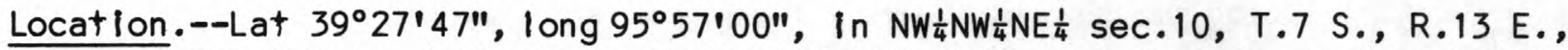
Jackson County, Hydrologic Unit 10270102, 160 feet downstream from bridge on State highway 16, $5.8 \mathrm{miles}$ southwest of Circleville, and at river mile 55.2. 
Drainage area.--49.3 square miles, almost all of which was measured on U.S. Geological Survey $7 \frac{1}{2}-m i n u t e$ quadrangle sheets.

Establishment.--March 1, 1964, by U.S. Geological Survey, Kansas District.

Gage. --Digital-punch recorder, 15-minute interval, driven by 50-foot-range bubble gage in $4 \times 4 \times 8$-foot steel-covered shelter on right bank at downstream side of the bridge. Auxillary equipment includes a graphical recorder.

Datum of gage is $1,094.58$ feet above NGVD of 1929 .

History.--Nov. 19, 1963 - Graphical A-35 recorder was started in operation. Gageheight record only collected through the winter months.

March 1,1964 - Regular gaging station established as part of a small streams investigation project.

Channel and control. -- The channel near the gage is in alluvium and is not stable. The low-water control is a gravel riffle about 200 feet downstream from the bridge. Both banks are wooded. Right-bank overflow starts at about 15 feet, but probably is not a rating factor because the channel runs along the right slde of the valley wall downstream from the gage. Left-bank overflow begins at about 16 feet, but may not be significant untll about 17 feet owing to a low dike along the left bank.

Beaver dams are a serious nuisance in low-flow perlods during the late summer, fall, and winter months.

Historic Floods.--Jesse Cowger (first house west of gage on north side) stated that the 1951 flood left a high-water mark at the edge of the pavement at the entrance to the fleld on the north side of the road west of the gage. This is the highest that Soldier Creek has ever been in his lifetime (born in 1908) or his father's IIfetime (Oscar Cowger, born 1883). The high-water mark was found to be 25.5 feet above gage datum and is considered to be of good rellablility.

Jesse Cowger has lived in Soldier Creek basin all of his life and at this location since 1945. A flood in the spring of 1945 was the second highest since he has been at this location and was about 21.1 feet. This mark was at the top board of a gate near his barn and is of falr rellabllity.

Both of these marks were on the upstream side of the bridge; however, $\mathrm{Mr}$. Cowger thought the fall through the bridge to be 0.5 foot or less.

Regulation and diversion.--No irrigation upstream is known. Beaver activity upstream occasionally may regulate low flow.

Maps.--Station is located on the Soldier Creek NW, Kansas, U.S. Geological Survey $7 \frac{1}{2}-m i n u t e$ quadrangle, topographic map. 
Selected climatic and physlographic basin parameters.--

$\begin{array}{lc}\text { Main-channel slope } & 10.8 \mathrm{ft} / \mathrm{ml} \\ \text { Slope at site } & 6.73 \mathrm{ft} / \mathrm{ml} \\ \text { Stream length } & 20.2 \mathrm{mi} \\ \text { Valley length } & 15.9 \mathrm{ml} \\ \text { Mean basin elevation } & 1,287 \mathrm{ft} \\ \text { Basin perimeter } & 24 \mathrm{ml} \\ \text { Storage } & 0.2 \text { percent } \\ \text { Forest } & 8.0 \text { percent } \\ \text { Mean annual precipitation } & 35.0 \mathrm{in} \\ \text { I 24, 2 } & 3.35 \mathrm{in} \\ \text { I 24,50 } & 6.60 \mathrm{in}\end{array}$

06889180 Soldier Creek near St. Clere, KS

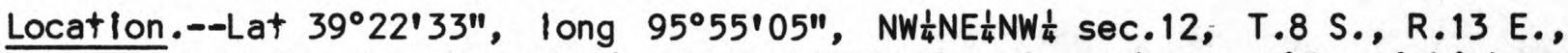
Jackson County, Hydrologic Unit 10270102, at upstream side of highway bridge, 7.8 miles east of St. Clere, and at river mile 44.5 .

Drainage area.--80 square miles, almost all of which was measured on U.S. Geological Survey $7 \frac{1}{2}-m i n u t e$ quadrangle sheets.

Establishment.--March 1, 1964, by U.S. Geological Survey, Kansas Distrlct.

Gage.--Digital-punch recorder, 15-minute interval, driven by 50-foot-range bubble gage in $4 \times 4 \times 8$-foot steel-covered shelter on right bank at downstream side of bridge. Auxillary equipment includes a graphical recorder. Datum of gage is 1,023.04 feet above NGVD of 1929 .

History.--Nov. 18, 1963 - Graphical recorder was started in operation. Gagehelght record only collected through winter months. March 1,1964 - Regular gaging station established as part of a small streams investigation project. Apr. 2, 1964 - Stage and rain gage digital recorders installed.

Channel and control.--The channel near the gage is in alluvium and is not stable. The low-water control is commonly formed by debris jams or remains of beaver dams at different locations within about 1,000 feet of the gage. Both banks are high and heavlly wooded. Right- and left-bank overflow starts at about 19 feet and extends about 1,000 feet on each bank over cultivated land at about 21 feet.

Historic Floods.--No flood information at this site prior to establishment of gage.

Regulation and diversion.--No consequentlal irrigation upstream is known. Beaver activity upstream occasionally causes regulation during low-flow perlods. 
Maps.--Station is located on Soldier Creek NW, Kansas, U.S. Geological Survey $7 \frac{1}{2}-m i n u t e$ quadrangle, topographic map.

Selected climatic and physlographic basin parameters.--

$\begin{array}{lc}\text { Main-channel slope } & 9.20 \mathrm{ft} / \mathrm{mi} \\ \text { Slope at site } & 3.60 \mathrm{ft} / \mathrm{mi} \\ \text { Stream length } & 31.2 \mathrm{ml} \\ \text { Valley length } & 22.6 \mathrm{mi} \\ \text { Mean basin elevation } & 1,250 \mathrm{ft} \\ \text { Basin perimeter } & 42 \mathrm{mi} \\ \text { Storage } & 0.2 \text { percent } \\ \text { Forest } & 8.0 \text { percent } \\ \text { Mean annual precipitation } & 35.1 \mathrm{in} \\ \text { I 24,2 } & 3.40 \mathrm{in} \\ \text { I 24,50 } & 6.65 \mathrm{in}\end{array}$

06889200 Soldier Creek near Della, KS

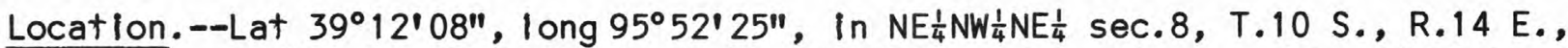
Shawnee County, Hydrologic Unit 10270102, at upstream slde of highway bridge, 5.1 miles upstream from Walnut Creek and $5.5 \mathrm{miles}$ southeast of Della, and at river mile 21.9.

Drainage area.--157 square miles, measured on 1953 Shawnee, Jackson, and Nemaha County highway maps. These planimetric maps $(1$ inch $=2$ miles $)$ have excellent drainage detalls and horizontal control.

Establishment.--October 1, 1958, by U.S. Geological. Survey, Kansas District.

Gage.--Digital-punch recorder, 15-minute interval, driven by 50-foot-range bubble gage in $4 \times 4 \times 8$-foot steel-covered shelter on right bank at downstream side of bridge. Auxillary equipment includes a graphical recorder.

Datum of gage is 931.34 feet above NGVD of 1929 .

History.--No other gages have been operated in the vicinity. The graphical recorder was installed September 22, 1958, but published records began October 1, 1958. March 20, 1964 - Digital recorder installed.

Channel and control.--The channel is a straight artificlal cut through clay where the channel was straightened when the bridge was bullt. Both banks are brush and willow covered. Right-bank overflow starts at about 16.5 feet over a cultivated field and may be one-half mile wide at 20-foot stage.

The left bank is about the same height as the right, below the junction of the old and new channels (dead water only in old channel), but the left flood plain ranges in width from zero to as much as a few hundred feet.

The low-water control is a rock low-water crossing for heavy equipment, approximately 1,500 feet below the gage. 
Historlc Floods.--Levels were run to the floor of the Clarence Anderson residence 600 feet upstream from gage and to a point just below the sill of the storm shelter. The floor, which was just reached by the 1951 flood, was at elevation 24.28 feet, and the shelter point, reached by the second highest flood, was at 24.03 feet. High-water marks painted on a shed door at the simecka residence $2.5 \mathrm{miles}$ north and $2 \mathrm{miles}$ west of gage were dated "6-21-51" for the highest mark, "9-4-51" for a mark 0.6 foot lower, and "7-19 [or 18, or 13] -51" for a mark 0.7 feet below the middle mark. Mr. Simecka stated that he neglected to mark the second highest flood, which his son thought occurred in 1942 (probably Aprll 23, 1944), and that the flood of "6-21-51" was the highest in his memory (since 1909). Another local resident stated that notable floods also occurred In 1915, 1903, and 1907, in order of descending magnitude.

The change in road fill and bridge in 1954 may have changed the highwater rating so that rellable figures of discharge can never be determined from past stage values.

Regulation and diversion.--Unknown amount of pumpage from stream for supplemental irrigation.

Maps.--Station is located on the Grove, Kansas, U.S. Geological Survey $7 \frac{1}{2}-m i$ nute quadrangle, topographic map.

Selected climatic and physlographic basin parameters.--

Main-channel slope
Slope at site
Stream length
Valley length
Mean basin elevation
Basin perimeter
Storage
Forest
. Mean annual precipitation
I 24,2
I 24,50

Main-channel slope

Slope at site

Stream length

Valley length

Mean basin elevation

Basin perimeter

Storage

Forest

I 24,2

124,50

\author{
$6.56 \mathrm{ft} / \mathrm{ml}$ \\ $3.52 \mathrm{ft} / \mathrm{mi}$ \\ $54.7 \mathrm{mi}$ \\ $35.1 \mathrm{mi}$ \\ $1,170 \mathrm{ft}$ \\ $70 \mathrm{~ms}$ \\ 0.2 percent \\ 8.0 percent \\ 35.2 in \\ 3.44 in \\ $6.70 \mathrm{in}$
}

06889500 Soldier Creek near Topeka, KS

Location.--Lat $39^{\circ} 06^{\prime} 00^{\prime \prime}$, long $95^{\circ} 43^{\prime} 27^{\prime \prime}$, In SW $\frac{1}{4} N W^{\frac{1}{4}} N W^{\frac{1}{4}}$ sec. 14, T.11 S., R. 15 E., Shawnee County, Hydrologic Unit 10270102, at downstream slde of highway bridge, 1.5 miles upstream from Halfday Creek, 2.1 miles northwest of Topeka, and at river mile 6.0 .

Drainage area.--290 square miles, all but 3 square miles of which was measured on U.S. Geological Survey $7 \frac{1}{2}-m i n u t e$ quadrangle sheets.

Establishment.--May 23, 1929, by U.S. Geological Survey, Kansas District. 
Gage.--Digital-punch recorder, 15-minute interval, driven by 50-foot-range bubble gage in $4 \times 4 \times 8$-foot steel-covered shelter on right bank at downstream side of bridge. Auxlliary equipment consists of a graphical recorder.

Datum of gage is 862.95 feet above NGVD of 1929 .

History.--May 23, 1929 - Chain gage installed at site 2 miles downstream from present site at different datum.

July 27, 1935 - Wire-welght gage installed at present site on bridge, now destroyed, at datum 4.00 feet higher.

June 17, 1958 - Water-stage recorder installed at present site on bridge, now destroyed, at datum 4.00 feet higher.

May 25, 1960 - Wire-welght gage installed at site 1.1 miles downstream at datum 1.79 feet lower.

June 9,1961-Bubble gage and graphical recorder installed at present site and datum.

April 15, 1964 - Digital-punch recorder added to existing equipment.

Channel and control.--The low-water channel is composed of mud and sand. The gage site is in a reach of manmade channel that is part of the flood protection system for North Topeka. Both banks are leveed in vicinity of gage and are not subject to overflow. The tops of levees at the gage are 34.5 feet above gage datum.

Historic Floods. --No flood information at this site prlor to establishment of gage.

Regulation and diversion.--Unknown amount of pumpage from stream for supplemental irrigation.

Maps.--Station is located on Topeka, Kansas, U.S. Geological Survey $7 \frac{1}{2}-m i n u t e$ quadrangle, topographic map.

Selected climatic and physlographic basin parameters.--

$\begin{array}{lc}\text { Main-channel slope } & 5.55 \mathrm{ft} / \mathrm{mi} \\ \text { Slope at site } & 3.54 \mathrm{ft} / \mathrm{mi} \\ \text { Stream length } & 71.1 \mathrm{ml} \\ \text { Valley length } & 46.9 \mathrm{mi} \\ \text { Mean basin elevation } & 1,120 \mathrm{ft} \\ \text { Basin perimeter } & 92 \mathrm{mi} \\ \text { Storage } & 0.3 \text { percent } \\ \text { Forest } & 8.15 \text { percent } \\ \text { Mean annual precipitation } & 35.2 \mathrm{in} \\ \text { I 24,2 } & 3.48 \mathrm{in} \\ \text { I 24,50 } & 6.75 \mathrm{in}\end{array}$




\section{APPENDIX B}

\section{Ground-Water Well Descriptions}

(Altitude of land surface given in feet above NGVD of 1929)

5-13E-9CDD. Observation well located 392 feet west of center of bridge at Goff streamf low gage. Well bored in alluvial deposits; $1.25-$ inch pipe installed to measure water-table altitudes. Depth (1963) 25.5 feet. Measuring point, top of pipe, 2.5 feet above land surface.

Altitude of land surface 1,312.75 feet.

Highest water level, 1.55 feet below LSD, Apr. 23, 1973.

Lowest water level, 15.10 feet below LSD, Nov. 2, 1964.

Records avallable 1963-76.

5-13E-9DCC. Observation well located 114 feet west of center of bridge at Goff streamflow gage. Well bored in alluvial deposits; 1.25 -inch pipe installed to measure water-table altitudes. Depth (1963) 29.4 feet. Measuring point, top of pipe, 3.5 feet above land surface.

Altitude of land surface 1,308.06 feet.

Highest water level, 6.70 feet below LSD, Apr. 22, 1969.

Lowest water level, 15.70 feet below LSD, Nov. 3, 1964.

Records avallable 1963-70, 1974.

5-13E-16ABB. Observation well located 42 feet east of center of bridge at Goff streamflow gage (destroyed 1970). Well bored in alluvial deposits; 1.25-inch pipe installed to measure water-table altitudes. Depth (1963) 34.0 feet. Measuring point, top of pipe, 2.0 feet above land surface.

Altitude of land surface 1,311.99 feet.

Highest water level, 6.73 feet below LSD, Apr. 22, 1969.

Lowest water level, 15.00 feet below LSD, Mar. 13, 1967.

Records avallable 1963-70.

5-13E-21CCD. Observation well located 332 feet east of center of bridge at Bancroft streamflow gage. Well bored in alluvial deposits; 1.25-inch pipe installed to measure water-table altitudes. Depth (1963) 29.0 feet. Measuring polnt, top of pipe, 3.4 feet above land surface.

Altitude of land surface 1,257.73 feet.

Highest water level, 7.28 feet below LSD, May 22, 1969.

Lowest water level, 15.33 feet below LSD, Mar. 13, 1967.

Records avallable 1963-70, 1974.

5-13E-28BBA. Observation well located 92 feet west of center of bridge at Bancroft streamflow gage. Well bored in alluvial deposits; 1.25-inch pipe installed to measure water-table altitudes. Depth (1963) 22.5 feet. Measuring point, top of pipe, 6.2 feet above land surface.

Altitude of land surface 1,250.51 feet.

Highest water level, 0.77 foot below LSD, Oct. 15, 1970.

Lowest water level, 10.50 feet below LSD, July 3, 1963.

Records avallable 1963-76. 
5-13E-28BBB. Observation well located 642 feet west of center of bridge at Bancroft streamflow gage. Well bored in alluvial deposits; $1.25-$ inch pipe installed to measure water-table altitudes. Depth (1963) 22.6 feet. Measuring polnt, top of plpe, 5.7 feet above land surface.

Altitude of land surface 1,253.11 feet.

Highest water level, 3.05 feet above LSD, Dec. 27, 1973.

Lowest water level, 7.51 feet below LSD, Oct. 2, 1964.

Records avallable 1963-76.

5-13E-33DDC. Observation well located 44 feet east of center of bridge at Soldier streamflow gage. Well bored in alluvial deposits; 1.25 -inch pipe installed to measure water-table altitudes. Depth (1963) 17.5 feet. Measuring point, top of pipe, 3.5 feet above land surface.

Altitude of land surface 1,221.02 feet.

Highest water level, 10.04 feet below LSD, June 15, 1964.

Lowest water level, dry.

Records avallable 1963-70, 1974.

5-13E-33DDC2. Observation well located 504 feet east of center of bridge at Soldier streamflow gage. Well bored in alluvial deposits; 1.25-inch pipe installed to measure water-table altitudes. Depth (1963) 21.0 feet. Measuring point, top of plpe, 4.0 feet above land surface.

Altitude of land surface 1,220.16 feet.

Highest water level, 0.43 foot above LSD, Mar. 23, 1973.

Lowest water level, 10.72 below LSD, July 24, 1963.

Records avallable 1963-76.

6-13E-4ABA. Observation well located 72 feet west of center of bridge at Soldier streamflow gage. Well bored in alluvial deposits; $1.25-i n c h$ pipe installed to measure water-table altitudes. Depth (1963) 22.5 feet. Measuring point, top of pipe, 5.7 feet above land surface.

Altitude of land surface 1,223.59 feet.

Highest water level, 13.39 feet below LSD, May 22, 1969.

Lowest water level, 16.38 feet below LSD, Nov. 17, 1969.

Records avallable 1963-73, 1974.

6-13E-4ABA2. Observation well located 444 feet west of center of bridge at Soldier streamflow gage. Well bored in alluvial deposits; $1.25-i n c h$ pipe installed to measure water-table altitudes. Depth (1963) 16.5 feet. Measuring point, top of pipe, 2.3 feet above land surface.

Alt itude of land surface 1,231.02 feet.

Highest water level, 12.63 feet below LSD, Jan. 19, 1970.

Lowest water level, dry.

Records avallable 1963-70, 1974. 
7-13E-3CDD. Observation well located 530 feet west of center of bridge at Circleville streamflow gage. Well bored in alluvial deposits; 1.25-inch pipe installed to measure water-table altitudes. Depth (1963) 23.0 feet. Measuring point, top of pipe, 3.4 feet above land surface. (Top of pipe, 1.1 feet above LSD in 1974.)

Altitude of land surface 1,132.90 feet.

Highest water level, 3.49 feet below LSD, May 21, 1969.

Lowest water level, 9.44 feet below LSD, Nov. 3, 1964.

Records avallable 1963-70, 1974.

7-13E-3DCC. Observation well located 66 feet west of center of bridge at Circleville streamflow gage. Well destroyed in 1970. Well bored in alluvial deposits; 1.00-inch pipe installed to measure watertable altitudes. Depth (1963) 36.5 feet. Measuring point, top of pipe, 4.0 feet above land surface.

Altitude of land surface 1,110.81 feet.

Highest water level, 1.56 feet below LSD, May 26, 1970.

Lowest water level, 12.18 feet below LSD, Apr. 13, 1968.

Records avallable 1963-70.

7-13E-3DCC2. Observation well located 122 feet east of center of bridge at Circleville streamflow gage. Well bored in alluvial deposits; 1.00-inch pipe installed to measure water-table altitudes. Depth (1963) 18.5 feet. Measuring point, top of pipe, 2.5 feet above land surface.

Altitude of land surface $1,110.28$ feet.

Highest water level, 9.68 feet below LSD, July 9, 1964.

Lowest water level, dry.

Records avallable 1963-70, 1974.

7-13E-3DCD. Observation well located 710 feet east of center of bridge at Circleville streamflow gage. Well bored in alluvial deposits; 1.25-inch pipe Installed to measure water-table altitudes. Depth (1963) 23.1 feet. Measuring polnt, top of pipe, 2.5 feet above land surface.

Altitude of land surface $1,122.68$ feet.

Highest water level, 11.38 feet below LSD, Mar. 26, 1973.

Lowest water level, dry.

Records avallable 1963-76.

8-13E-1CCC. Observation well located 1,080 feet west of center of bridge at St. Clere streamflow gage. Well bored in alluvial deposits; 1.00-inch pipe installed to measure water-table altitudes. Depth (1963) 14.0 feet. Measuring point, top of pipe, 3.0 feet above land surface.

Altitude of land surface 1,043.66 feet.

Highest water level, 1.28 feet below LSD, June 18, 1969.

Lowest water level, 13.49 feet below LSD, Nov. 24, 1964.

Records avallable 1963-70, 1974. 
8-13E-1CCD. Observation well located 556 feet west of center of bridge at St. Clere streamflow gage. Well bored in alluvial deposits; 1.00-inch pipe installed to measure water-table altitudes. Depth (1963) 42.2 feet. Measuring point, top of pipe, 4.0 feet above land surface.

Altitude of land surface 1,045.05 feet.

Highest water level, 4.60 feet below LSD, June 18, 1969.

Lowest water level, 14.83 feet below LSD, Apr. 15, 1964.

Records available 1963-70, 1974.

8-13E-1CCD2. Observation well located 98 feet west of center of bridge at St. Clere streamflow gage. Well bored in alluvial deposits; $1.00-i n c h$ pipe installed to measure water-table altitudes. Depth (1963) 43.5 feet. Measuring point, top of pipe, 3.5 feet above land surface.

Altitude of land surface 1,043.14 feet.

Highest water level, 4.25 feet LSD, Nov. 24, 1964.

Lowest water level, 15.22 feet below LSD, Apr. 15, 1964.

Records avallable 1963-70, 1974.

8-13E-ICCD3. Recording observation well located 410 feet west of center of bridge at St. Clere streamflow gage. Well bored in alluvial deposits; $1.25-$ inch pipe installed to measure water-table altitudes. Depth (1963) 36.2 feet. Measuring point, top of pipe, (1964 to November 1970) 3.0 feet above land surface, (November 1970 to present) 1.7 feet above land surface.

Altitude of land surface 1,042.76 feet.

Highest water level, 0.30 foot below LSD, Oct. 17, 1968.

Lowest water level, 13.34 feet below LSD, May 26, 1964.

Records avallable 1964-76.

8-13E-1CCD4. Recording observation well located 80 feet west of center of bridge at St. Clere streamflow gage. Well bored in alluvial deposits; 5-inch pipe installed to measure water-table altitudes. Depth (1963) 36.2 feet. Measuring point, top of pipe, 3.6 feet above land surface.

Altitude of land surface 1,043.00 feet.

Highest water level, 4.02 feet below LSD, June 21, 1967.

Lowest water level, 19.97 feet below LSD, Oct. 15, 1968.

Records avallable 1964-69, 1976.

8-13E-1CCD5. Observation well located 305 feet west of center of bridge at St. Clere streamflow gage. Well bored in alluvial deposits; $1.25-i n c h$ pipe installed to measure water-table altitudes. Depth (1974) 39.6 feet. Measuring point, top of pipe, 1.4 feet above land surface.

Altitude of land surface 1,042.77 feet.

Highest water level, 3.95 feet below LSD, June 18, 1969.

Lowest water level, 13.58 feet below LSD, Mar. 13, 1967.

Records avallable 1965-70, 1974. 
8-13E-1CCD6. Observation well located 275 feet west of center of bridge at St. Clere streamflow gage. Well bored in alluvial deposits; $1.25-$ inch pipe installed to measure water-table altitudes. Depth (1974) 36.2 feet. Measuring polnt, top of pipe, 1.6 feet above land surface.

Altitude of land surface 1,042.74 feet.

Highest water level, 3.56 feet below LSD, June 18, 1969.

Lowest water level, 13.12 feet below LSD, Feb. 13, 1967.

Records avallable 1965-70, 1974.

8-13E-ICDC. Observation well located 62 feet east of center of bridge at St. Clere streamflow gage. Well destroyed as of 1974. Well bored in alluvial deposits; 1.00-inch pipe installed to measure watertable altitudes. Depth (1963) 27.0 feet. Measuring point, top of pipe, 3.0 feet above land surface.

Altitude of land surface 1,043.77 feet.

Highest water level, 2.72 feet below LSD, June 20, 1967.

Lowest water level, 18.83 feet below LSD, Jan. 4, 1965.

Records avallable 1963-70.

8-13E-1CDC2. Observation well located 662 feet east of center of bridge at St. Clere streamflow gage. Well destroyed as of 1965. Well bored in alluvial deposits; 1.00-inch pipe installed to measure watertable altitudes. Depth (1963) 36.5 feet. Measuring point, top of pipe, 3.0 feet above Iand surface.

Altitude of land surface 1,044.26 feet.

Highest water level, 7.85 feet below LSD, Sept. 24, 1963.

Lowest water level, 16.48 feet below LSD, Apr. 15, 1964.

Records avallable 1963-65.

8-13E-1DCC. Observation well located 1,226 feet east of center of bridge at St. Clere streamflow gage. Well bored in alluvial deposits; 1.00-inch pipe installed to measure water-table altitudes. Depth (1963) 30.1 feet. Measuring point, top of pipe, 3.5 feet above land surface.

Altitude of land surface 1,043.58 feet.

Highest water level, 7.12 feet below LSD, July 24, 1963.

Lowest water level, 18.81 feet below LSD, Apr. 15, 1964.

Records avallable 1963-70, 1974.

10-14E-5CDC. Observation well located 2,157 feet west of center of bridge at Della streamflow gage. Well bored in alluvial deposits; 1.00-inch pipe installed to measure water-table altitudes. Depth (1963) 23.5 feet. Measuring point, top of pipe, 3.0 feet above land surface.

Altitude of land surface 956.52 feet.

Highest water level, 0.19 foot below LSD, Nov. 2, 1967.

Lowest water level, 7.16 feet below LSD, Mar. 14, 1967.

Records avallable 1963-70, 1974. 
10-14E-5DCD. Observation well located 5 feet east of center of bridge at Della streamflow gage. Well destroyed as of 1968. Well bored in alluvial deposits; $1.25-i n c h$ pipe installed to measure water-table altitudes. Depth unknown. Measuring point, top of pipe, used 0.0 feet above land surface since sandy streambed is subject to frequent shifting.

Altitude of land surface 937.73 feet.

Highest water level, 0.05 foot below LSD, Apr. 18, 1968.

Lowest water level, 5.53 feet below LSD, Oct. 13, 1966.

Records avallable 1965-68.

10-14E-5DDD. Observation well located 1,400 feet east of center of bridge at Della streamflow gage. Well destroyed in 1964. Well bored in alluvial deposits; 1.00-inch pipe installed to measure water-table altitudes. Depth 31.6 feet. Measuring polnt, top of plpe, 4.7 feet above land surface.

Altitude of land surface 958 feet.

Highest water level, 11.68 feet below LSD, Sept. 24, 1963.

Lowest water level, 14.88 feet below LSD, Dec. 5, 1963.

Records avallable 1963.

10-14E-8AAB. Observation well located 150 feet east of center of bridge at Della streamflow gage. Well bored in alluvial deposits; $1.00-i n c h$ pipe Installed to measure water-table altitudes. Depth (1963 - 24.3 feet) 35.5 feet measured in 1974. Measuring point, top of pipe, 3.0 feet above land surface.

Altitude of land surface 949.77 feet.

Highest water level, 3.90 feet below LSD, June 26, 1967.

Lowest water level, 18.03 feet below LSD, Mar. 13, 1964.

Records avallable 1963-70, 1974.

10-14E-8AAB2. Observation well located 660 feet east of center of bridge at Della streamflow gage. Well bored in alluvial deposits; 1.00-inch pipe Installed to measure water-table altitudes. Depth (1963) 26.1 feet. Measuring point, top of pipe, 3.0 feet above land surface.

Alt itude of land surface 957.78 feet.

Highest water level, 9.58 feet below LSD, May 20, 1969.

Lowest water level, 18.25 feet below LSD, Apr. 11, 1967.

Records avallable 1963-70, 1974.

10-14E-8ABA. Observation well located 220 feet west of center of bridge at Della streamflow gage. Well bored in alluvial deposits; $1.00-i n c h$ pipe installed to measure water-table altitudes. Depth (1963) 48.3 feet. Measuring polnt, top of pipe, 3.0 feet above land surface.

Altitude of land surface 947.76 feet.

Highest water level, 0.23 foot below LSD, June 26, 1967.

Lowest water level, 14.31 feet below LSD, Mar. 13, 1964.

Records avallable 1963-72, 1974. 
10-14E-8ABB. Observation well located 957 feet west of center of bridge at Della streamflow gage. Well bored in alluvial deposits; $1.00-$ inch pipe installed to measure water-table altitudes. Depth (1963) 38.8 feet. Measuring point, top of pipe, 3.3 feet above land surface.

Altitude of land surface 952.85 feet.

Highest water level, 0.85 foot below LSD, June 15, 1969.

Lowest water level, 13.43 feet below LSD, Mar. 14, 1967.

Records avallable 1963-70, 1974. 
